\title{
THE CURRENT STATE OF SAFETY WITHIN THE WET LABORATORIES OF A CANADIAN ACADEMIC INSTITUTION
}

By

\author{
Helene-Rosina Ayi, \\ Bachelor of Applied Science, \\ Ryerson University, 2014
}

A thesis presented to Ryerson University

in partial fulfilment of the requirements for the degree of Master of Applied Science

in the program of

Environmental Applied Science and Management

Toronto, Ontario, Canada, 2017

(C) Helene-Rosina Ayi 2017 


\section{Author's Declaration}

I hereby declare that I am the sole author of this thesis. This is a true copy of the thesis, including any required final revisions, as accepted by my examiners. I authorize Ryerson University to lend this thesis to other institutions or individuals for the purpose of scholarly research.

I further authorize Ryerson University to reproduce this thesis by photocopying or by other means, in total or in part, at the request of other institutions or individuals for the purpose of scholarly research. I understand that my thesis may be made electronically available to the public. 


\title{
THE CURRENT STATE OF SAFETY WITHIN THE WET LABORATORIES OF A CANADIAN ACADEMIC INSTITUTION
}

\author{
Helene-Rosina Ayi \\ Master of Applied Science, 2017 \\ Environmental Applied Science and Management, Ryerson University
}

\begin{abstract}
Working in an academic laboratory (lab) often involves handling hazardous substances (Shariff \& Norazahar, 2012). These substances are dangerous due to their toxic, flammable, explosive, carcinogenic, pathogenic or radioactive properties (Furr, 2000). Therefore, it is crucial that those working in these environments do so safely. Recently, many researchers and students from various universities in the U.S. and globally have suffered severe injuries and fatalities from lab accidents. For example, in 2008 a lab fire at the University of California Los Angeles led to the death of a student(Van Noorden, 2011). Following this and other similar accidents that transpired afterwards, an international study was conducted to understand the state of safety within the wet labs of today's universities(Van Noorden, 2013a). The findings revealed numerous safety gaps and an overall lack of a strong and positive safety culture within the labs (Benderly, 2013;and Schröder, Huang, Ellis, Gibson, \& Wayne, 2016).

Since the majority of the accidents and study reports were predominantly from the U.S., it is unknown if the same safety gaps and risks also exist in the wet labs of Canadian universities. Therefore, this research study examined the state of safety within the wet laboratories of a medium-sized Canadian university. This was achieved by: 1) conducting an inventory of the labs' hazardous substances to identify their labeling and storage conditions, 2) inspecting the labs to identify potential hazards or risky conditions, and 3) surveying lab personnel to understand how safety is perceived and practiced. The results show several safety deficiencies and a negative perception on certain safety elements among the lab personnel. As in universities in the U.S. there is an overall need to enhance the current culture of safety at this Canadian university.
\end{abstract}




\section{Acknowledgments}

I would like to thank God for my academic supervisor, Dr. Chun-Yip Hon, who tremendously encouraged and supported me, as well as provided knowledgeable input throughout the learning process of this thesis. I am immensely grateful for his scientific and research expertise that helped strengthen the caliber of this thesis as well as my research writing skills. I am also thankful for the time he spent to read and provide in-depth constructive and timely feedback the thesis chapters. I would like to acknowledge the opportunity to work as a Graduate Assistant for two of his undergraduate courses. This opportunity helped alleviate a lot of my financial research burdens. It was

truly an honor to be his master's student. I am more than certain that future master's and/or PhD students who get the privilege to be supervised by Dr. Hon will not regret the experience and will gain from it. So once again, thank you Dr. Hon. I could not have reached this place without your expertise, guidance, and genuine support.

I am extremely grateful for the tremendous help of my research assistants, Ailiya Sayeed, Paul Somal, Daniel Yej Jeong, and Shereen Salama. These were third and fourth year students from the School of Occupational and Public Health who volunteered their time to help collect some of my thesis data i.e. the chemical inventory and safety inspection data. Without their assistance, I probably would have still been collecting those data.

I would like to thank the School of Occupational and Public Health, which gave me numerous GA and Exam Invigilator positions. These opportunities also helped alleviate some of my academic financial burdens. I would like to specifically extend my thanks and acknowledgements to Christopher Bentley, from the School, for his assistance with some aspects of my thesis. Also, I would like to specifically thank Dr. Ronald Pushchak, Dr. Corinne Ong, Dr. Richard Meldrum, Dr. Anne Harris, Dr. Peter Strahlendorf, Dr. Eric Liberda, Darlene Bileckyj, and Sheila Jones for their encouragement during this thesis process.

I am very grateful to Dr. James Scott from the University of Toronto, for allowing me to freely use his lab (Sporometrics Inc) to collect and analyse some of my thesis data.

I am tremendously grateful to God for my parents, who encouraged me through their prayers and demonstrated a lot of patience, understanding, and support towards me when I was discouraged. My thanks are also extended to all my friends, who helped edit my thesis. I am very grateful for their constructive criticisms which helped strengthened my writing skills. 


\section{Table of Contents}

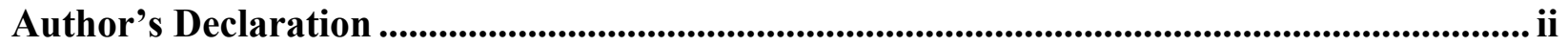

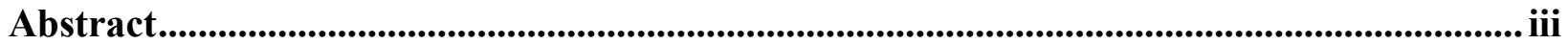

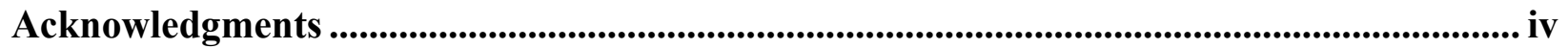

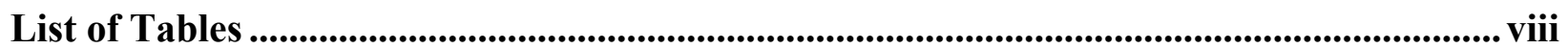

List of Figures.................................................................................................................................... ix

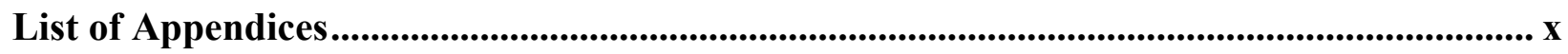

Glossary of terms .......................................................................................................................... xi

Chapter 1- Introduction ........................................................................................................... 1

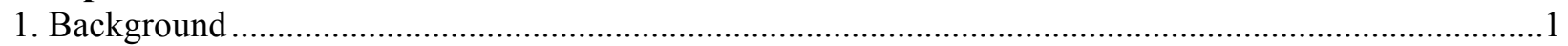

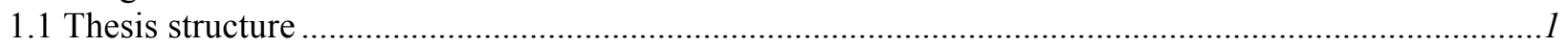

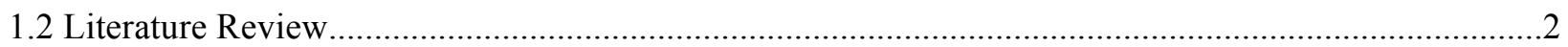

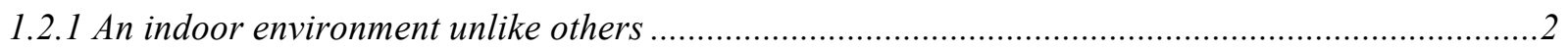

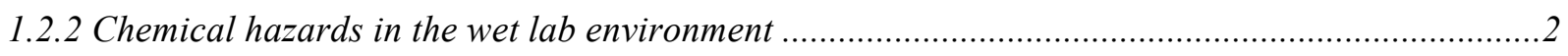

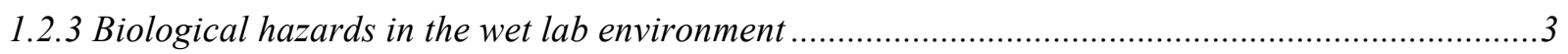

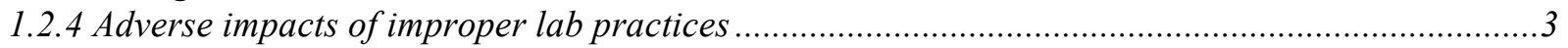

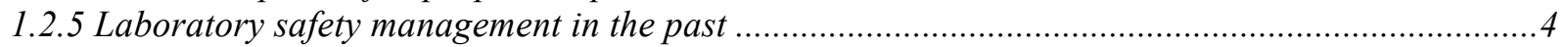

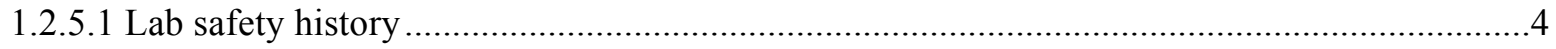

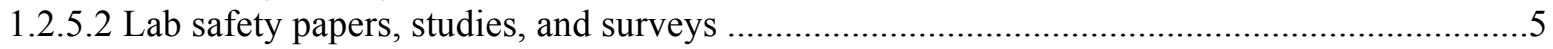

1.2.6 Regulations and guidelines for lab substances .................................................................. 6

1.2.6.1 Biological agents: Canadian regulations and guidelines................................................. 7

1.2.6.2 Chemical agents: Canadian regulations and guidelines .....................................................

1.2.7 The current state in today's academic wet labs .......................................................................

1.2.7.1 Safety conditions in today's university wet labs .............................................................

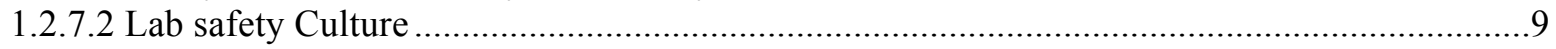

1.2.7.2.1 Safety culture within academia: a need for improvement .......................................10

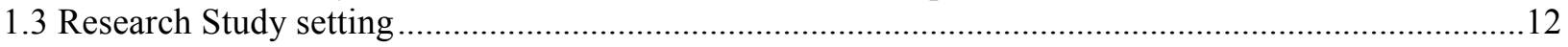

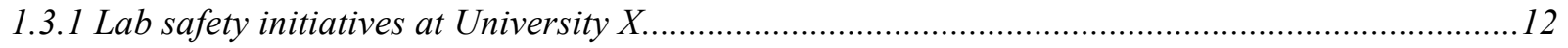

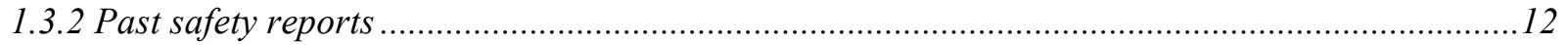

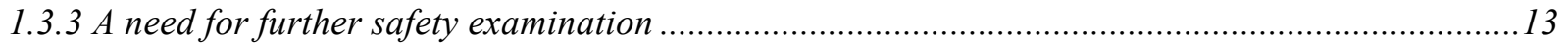

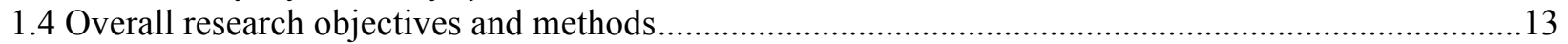

Chapter 2- Academic lab chemical management and workers' risk perception ................... 18

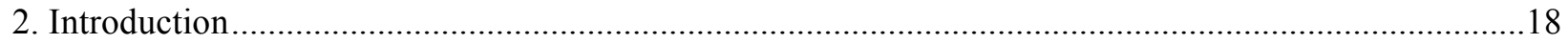

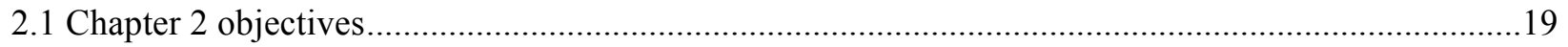

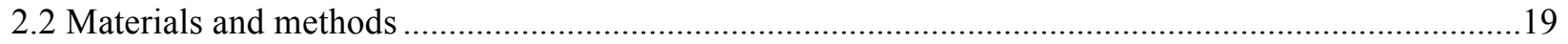

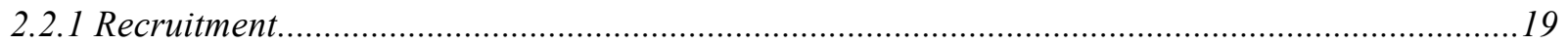

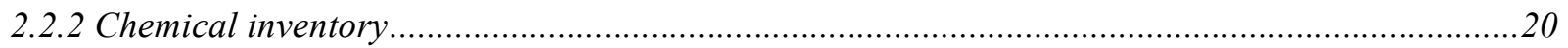

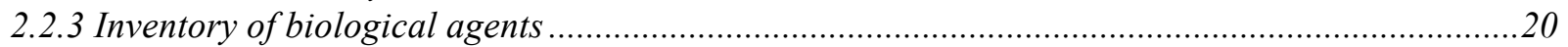

2.2.4 Hazard level of the chemicals and biological agents ............................................................20

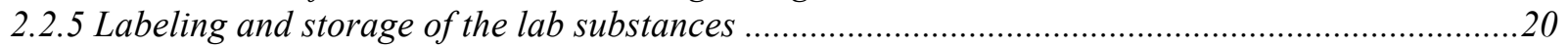

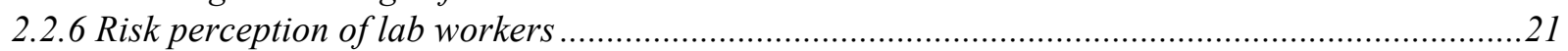

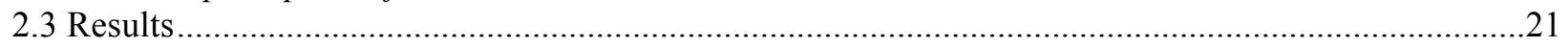

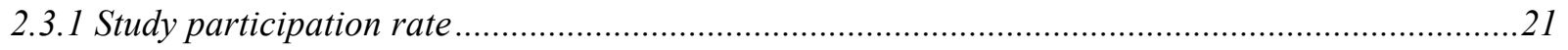

2.3.2 Inventories: Chemicals within the University's laboratories..................................................21

2.3.3 Inventories: labeling and storage condition of the University's lab chemicals.........................22

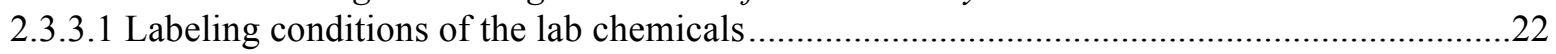




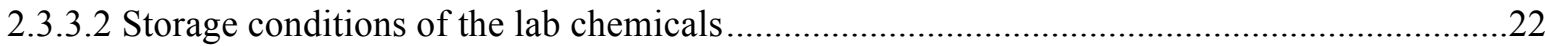

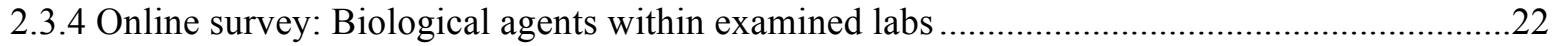

2.3.5 Online survey: Lab risk perception among examined lab personnel ......................................23

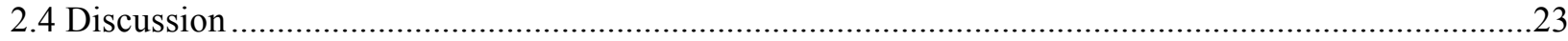

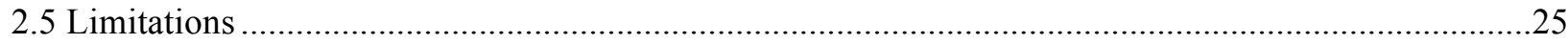

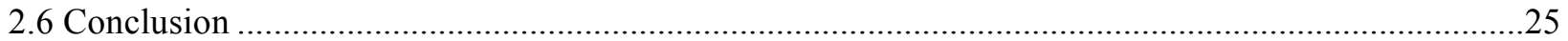

Chapter 3- Identification of safety deficiencies within examined Chemistry and Biology wet

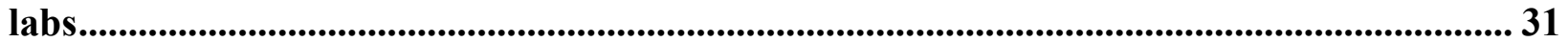

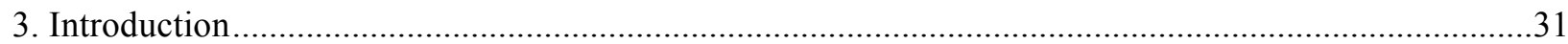

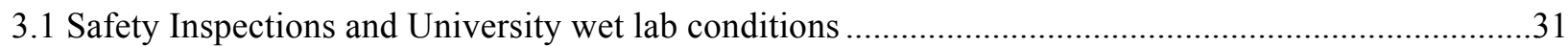

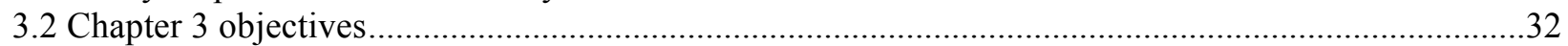

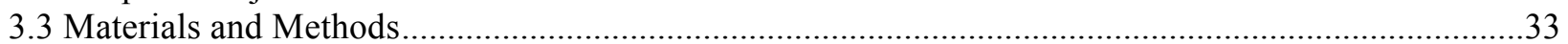

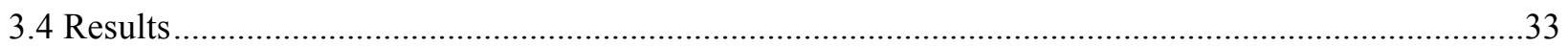

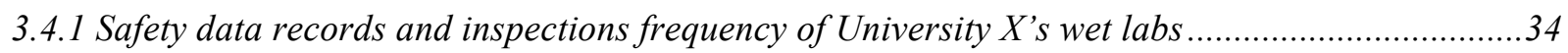

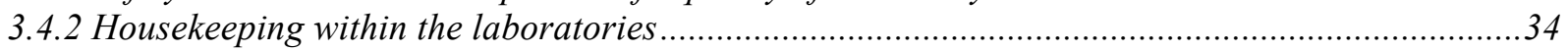

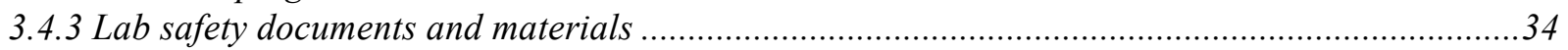

3.4.4 Lab Safety Devices, Emergency and Proper Protective Equipment(PPE) ................................34

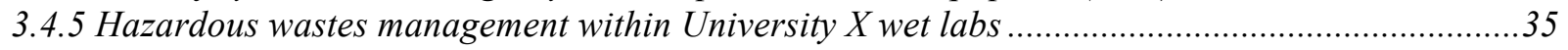

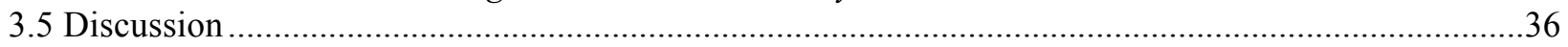

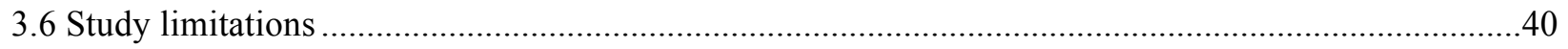

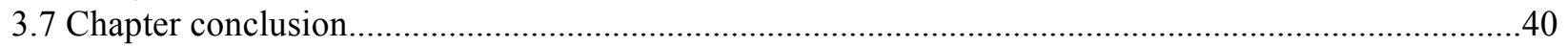

Chapter 4- Safety attitudes and practices of University X Lab Personnel ............................. 46

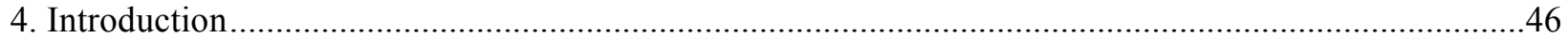

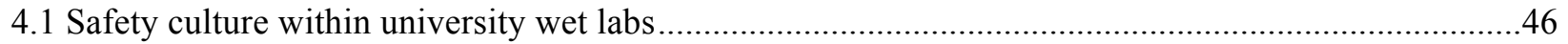

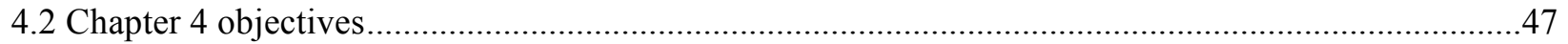

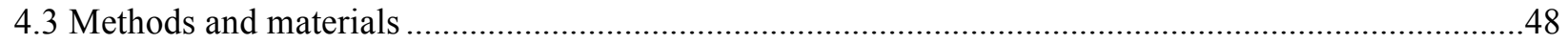

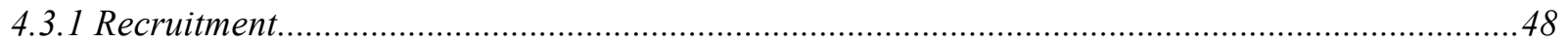

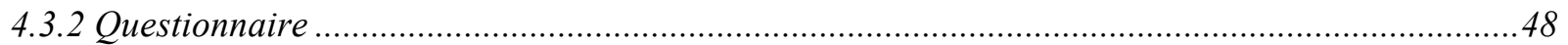

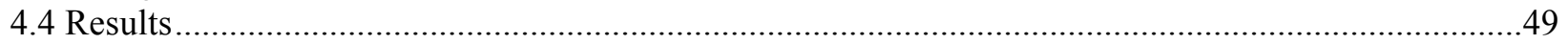

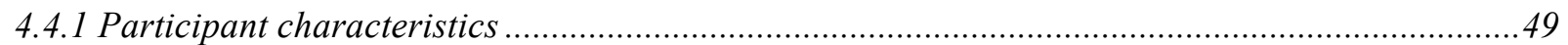

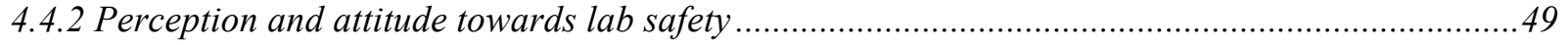

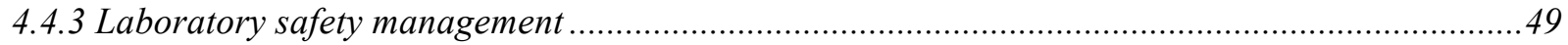

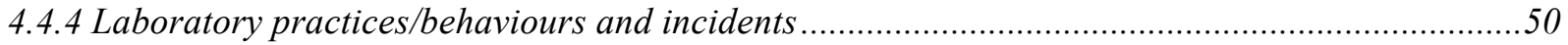

4.4.5 Safety culture: a comparison between University $X$ and other universities...............................51

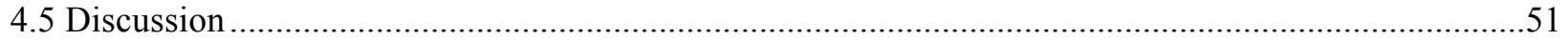

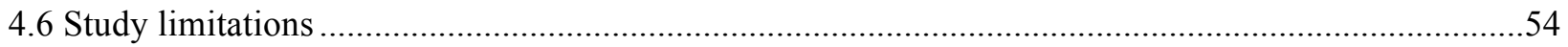

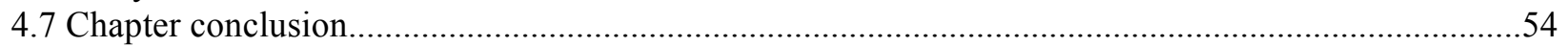

Chapter 5- Conclusion and Recommendations......................................................................... 64

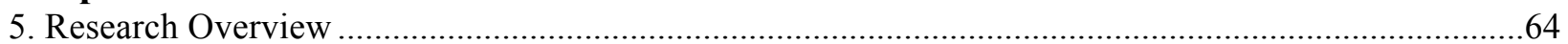

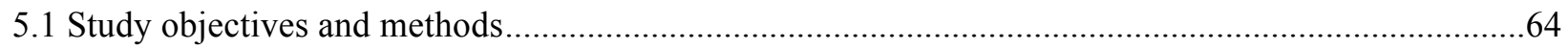

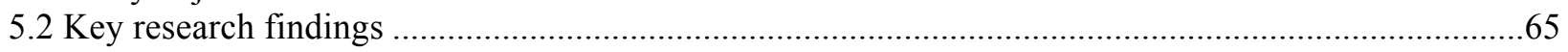

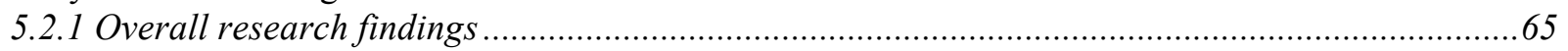

5.2.2 Hazardous chemicals within some wet labs at University X (Chapter 1) ..................................65

5.2.3 Safety deficiencies found in some wet labs at University X (Chapter 2) .....................................65

5.2.4 Safety attitudes and practices among University X's lab personnel (Chapter 3) .......................66

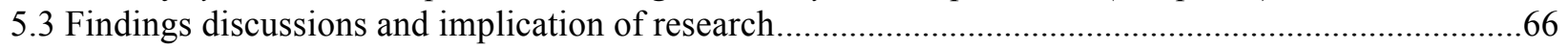

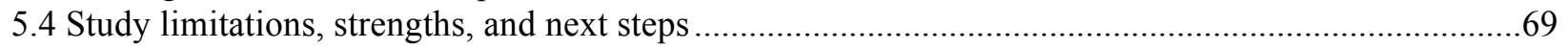

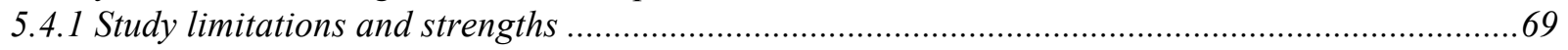

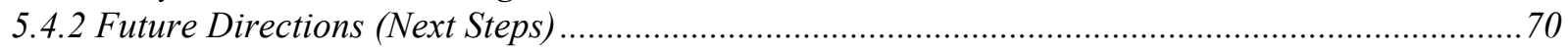


5.5 Conclusions

5.6 Recommendations

5.6.1 Enhance employer and superiors' awareness of their OHSA responsibilities.

5.6.2 Enhancement of compliance with lab safety rules and best practices

5.6.3 An overall improvement of safety culture within the labs

5.6.4 An overall better-integrated IRS system within University's lab environments...........................74

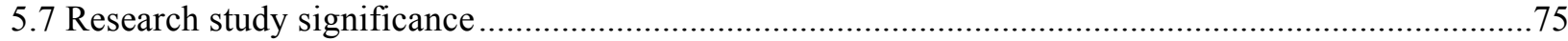

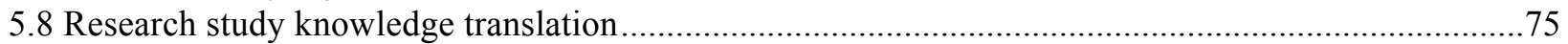

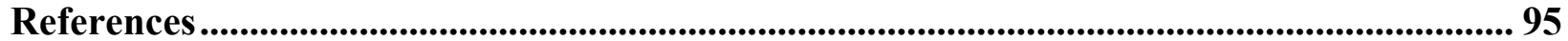




\section{Chapter 1}

\section{List of Tables}

Table 1.1: Summary of WHMIS hazard classes and corresponding symbols 15

Table 1.2: Accidents cases within the wet labs of universities in the U.S. and around the world

\section{Chapter 2}

Table 2.1: Chemicals commonly present within University X's wet labs and their hazard class(s) ..........27

Table 2.2: Examples of select inventoried chemicals that were incompatibly stored

\section{Chapter 3}

Table 3.1: Examples of essential lab safety items (safety manuals, equipment, and safety devices).........41

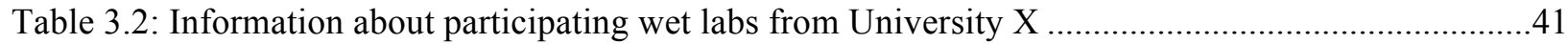

Table 3.3: Housekeeping within University x's chemistry and biology wet labs ..................................42

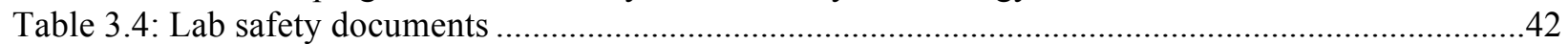

Table 3.5: Availability and safety condition of Personal Protective Equipment and Safety Devices within

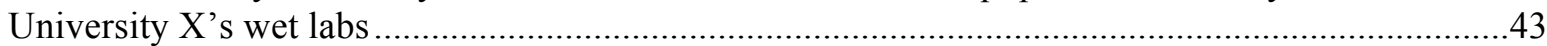

Table 3.6: Hazardous Waste Management within University x's wet labs ............................................44

\section{Chapter 4}

Table 4.1: Survey participants characteristics

Table 4.2: Perception and attitudes towards safety among University X's lab researchers and students ...57

Table 4.3a: Lab safety management based on responses from all participants ........................................58

Table 4.3b: Lab safety management based on responses from lab superiors ….....................................59

Table 4.4: Lab safety practices within University X …........................................................................60 


\section{List of Figures}

\section{Chapter 1}

Figure 1A. Example of a WHMIS supplier label

Figure 1B. Example of a WHMIS workplace label

\section{Chapter 2}

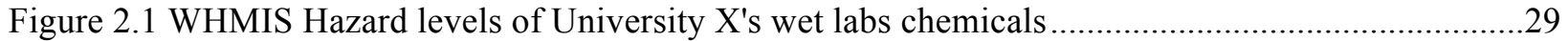

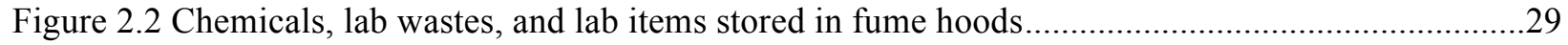

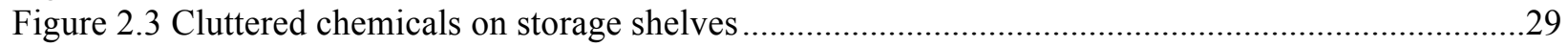

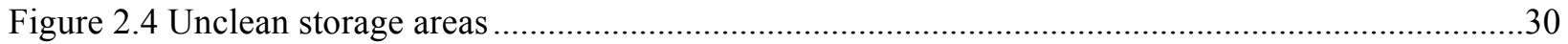

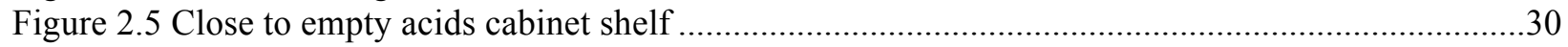

\section{Chapter 3}

Figure 3.1. Laboratory safety deficiencies identified after 2016 safety inspections

\section{Chapter 4}

Figure 4.1 What was the nature of your injury or injuries? Check all that apply ...................................61

Figure 4.2 Lab safety improvement barriers: medium-sized Canadian university vs 2012 study

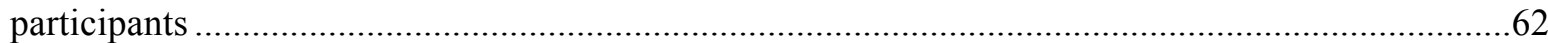

Figure 4.3 Lab safety culture: medium-sized Canadian University vs other 2012 study participants ........63 


\section{List of Appendices}

\section{Chapter 2}

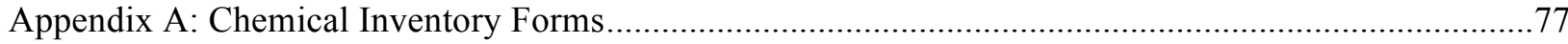

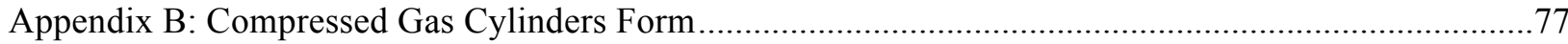

\section{Chapter 3}

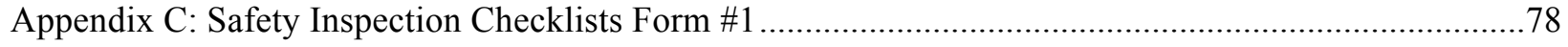

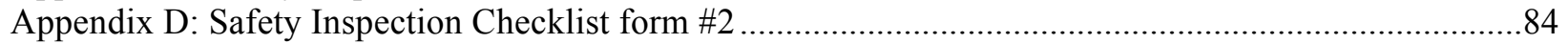

Appendix E: Excerpt from University X’s Workplace Inspection Program ...........................................86

\section{Chapter 4}

Appendix F: Survey ... 


\section{Glossary of terms}

Biosafety containment levels: These provide lab workers, who handle infectious or biohazardous agents, with the necessary containment required to safely handle these agents. They help protect lab workers, the environment, and the general public from exposure to the hazards and risk associated with the agents. There are four levels of containment, each classified in a graded fashion. For example, biological agents that pose low health and safety risks are handled in the first containment level; while those that pose very high health and safety risks are handled in the fourth containment level. (Virginia University, n.d.; \& McLeod, 2010)

Chemical fume hoods: These are ventilated enclosures that are designed to contain and exhaust toxic fumes or vapours, mists, and particulate matter generated in the hood from lab operations. Essentially, these are safety devices that are used in labs to contain hazardous substances. These are meant to protect workers from breathing contaminated or polluted air generated from lab experiments. A properly working and appropriately used fume hood can reduce or even eliminate exposure to volatile liquids etc. (Lab Crafters, n.d.)

Hazard: This is anything that causes or can cause harm. It is "often associated with an agent, condition, or activity (a natural phenomenon, a chemical, a mixture of substances, a process involving substances, a source of energy, or a situation or event) that if left uncontrolled, could result in an injury, illness, loss of property, or damage to the environment". Essentially, "hazards are intrinsic properties of agents, conditions, or activities". Hazard must not be confused with the term 'Risk' as this refers to the probability for harm to occur. (American Chemical Society, 2015; and Canadian Centre for Occupational Health and Safety (CCOHS), 2009b)

Hazard classes: These are a way of grouping chemicals or products that share the same inherent hazard properties. (CCOHS, 2010; Government of Canada, 2016c)

Health Belief Model (HBM): This is a model developed in the 1950s by psychologists (Hochbaum, Rosenstock, and Kegels, who were working with the U.S. Public Health Services at the time). It explains and predicts people's health and safety behaviours, by focusing on their attitudes and behaviour. The HBM proposes that people will respond positively to messages promoting health and safety behaviours if they believe that: 1) they are at risk of developing an unhealthy condition, 2) the risk is very serious and that the consequences or outcomes are undesirable, 3) the risk will be reduced or minimized by specific behaviour change, and 4) there are available tools to help them make those changes and that the barriers to the behaviour change can be overcome and managed.

(Oben, n.d.; and University of Twente, n.d.) 
Incompatible substances: These are substances that should not be stored beside each other (i.e. acids and bases), because contact with each other could result in dangerous reactions which may lead to fires, explosions, injuries etc. (University of Iowa, 2012)

Internal Responsibility System (IRS): This is the principle under which the Occupational Health \& Safety Act (OHSA) in Ontario operates. It means that everyone within an organization has a unique role to play in ensuring and maintaining their workplace safe and healthy for all. (Ministry of Labour, 2015)

Inventory (Chemical Inventory): This is a database that lists all the chemicals handled and stored in the laboratory environment. Essentially, it is a management system that helps keep track of the chemicals or other hazardous products that are present in the lab, as well as the condition in which they are present. It is a very useful tool for an effective chemical emergency response. (US EPA, 2016; and US National Research Council, 2011)

Pictograms: These are graphical images that indicate to a lab worker the hazards associated with a chemical. (CCOHS, 2016)

Personal Protective Equipment (PPE): These include lab coat, safety shoes, eye-protection, gloves etc. They are worn by workers to help minimize exposure to the hazards associated with their lab work. PPE is the last line of defense when it comes to controlling hazardous exposures. (Ministry of Labour, 2015)

Research labs: These are spaces utilized by skilled and experienced individuals to carry out research for the furthering of scientific knowledge. (Hofstein \& Lunetta, 2004; and Watch, Tolat, \& McNay, 2010).

Risks: This refers to the probability that harm or an adverse consequence will occur. It also accounts for the severity of the harm or adverse consequence should it occur. This should not be confused with the term 'hazard'. (American Chemical Society; \& CCOHS, 2017)

Risk Assessment: The process used to identify hazards, analyze or evaluate the risk associated with hazards, and provides or determines ways in which identified hazards can be controlled or removed. (CCOHS, 2009a)

Safety culture: This consists of shared beliefs, practices/behaviours, and attitudes regarding safety that exist within a workplace. It is the atmosphere that is created by those beliefs, attitudes, etc., which shape the behaviours in the workplace. (Gutiérrez, Emery, Whitehead, \& Felknor, 2013)

Safety Data Sheets (SDSs): These are summary documents that inform lab personnel about the hazards and risks potentially associated with their work chemicals. The hazard information provided in these summary documents are 
more detailed than what is provided by a standard supplier or workplace label. They were formerly referred to as 'Material Safety Data Sheets”. ( CCOHS, 2016b)

Safety Inspections: They examine the safety condition of a work environment. Specifically, they are carried out to identify existing and potential hazards as well as other unsafe lab conditions. From their results, corrective actions are provided for any hazard or safety deficiency identified in the workplace. When these corrective actions are addressed, they help minimize or prevent the occurrence of work-related injuries and illnesses. (CCOHS, 2009)

Signal words: These are found on chemical labels to alert end user about the degree or level of hazard associated with the chemical. The words used are 'warning', assigned to chemicals associated with less severe hazards, and 'danger', assigned to chemicals that are high-risk hazards. (CCOHS, 2016b)

Supervisor: According to the Occupational Health and Safety Act, this is "anyone who has charge of a workplace or authority over a worker" (Government of Ontario, 2014c). In an academic setting, this includes deans, chairs, and principal investigators as people report to them.

Teaching labs: These are academic spaces in which students or other inexperienced individuals gain hands-on practical scientific knowledge as well as lab experience. (Hofstein \& Lunetta, 2004; Watch, Tolat, \& McNay, 2010).

Wet laboratories: These are indoor environments equipped with the necessary plumbing, ventilation, and equipment to allow for hands-on scientific research and experiment which involve the use of liquid solutions or volatile phases. These are the typical chemistry and biology laboratories. (Whole Building Design Guide, 2010) Workplace Hazardous Materials and Information System (WHMIS): This is a Canada-wide regulation which asks that all workers and employers are informed about the hazards associated with their respective work substances or products. Under WHMIS hazard information is disseminated through labels, Safety Data Sheets (formerly known as Material Safety Data Sheets), and the implementation of training programs. (Ministry of Labour, 2009)

Working alone: This is when someone cannot be seen or heard by others while working. While it is not always harmful to work alone, but in circumstances when hazardous substances are handled it is dangerous and not a best practice. (CCOHS, 2014) 


\section{Chapter 1- Introduction}

\section{Background}

Academic laboratories are commonly utilized for teaching and research purposes (Gutiérrez, Emery, Whitehead, \& Felknor, 2013; and Artdej, 2012 ). These environments have played a significant role in the betterment of society. In fact, many of the world's most important scientific discoveries (i.e. discovery of insulin, the germ theory, discovery of vaccines, etc.) were made in university laboratories (National Research Council, 2014; Bliss, 2015; Twisselmann, 2003; \& Chemical Heritage Foundation, 2016). However, these workplaces pose considerable risk to workers, students, and the outdoor environment (Tenkate, McBride, \& Richard, 2001; Gutiérrez et al., 2013; and Langerman, 2009). Unlike some workplaces, operating and/or studying in university labs generally involves the handling of various chemical and/or biological substances. Many of these substances are hazardous to varying degrees (Adane \& Abeje, 2012). Therefore, the manner in which they are handled is crucial. If they are unsafely managed (handled, stored, and disposed of), the health and safety of lab workers, students, and the general public may be adversely affected.

Recently, numerous accidents have been reported from the wet laboratories of many universities in several countries (Johnson and Kemsley, 2011; Eldridge, 2012; and Jia, 2016). As a result, the state of safety within university wet labs is questioned by academic and safety professionals (Eldridge, 2012). In fact, the literature suggests that more attention should be directed toward examining the safety conditions in today's academic labs (Richards-Babb, Bishoff, Carver, Fisher, \& Robertson-Honecker, 2010; and Johnson \& Kemsley, 2011). Therefore, this thesis focuses on addressing the following research question: what is the state of safety within the wet laboratories of a Canadian university?

\subsection{Thesis structure}

This thesis is organized around 5 chapters. Three of the chapters address the overall research objectives by focusing on a specific safety element: Chapter 2- chemical inventory, Chapter 3 - safety inspection, and Chapter 4 safety culture. Although each of these chapters is meant to stand alone, they are not independent and some interlinked concepts can be found throughout the thesis. Bookending the three chapters is an introduction of lab safety literature and a chapter which summarizes the findings of the entire study as a whole. For a clear and easy read, the Canadian university studied in this research was given the name "University X". This name is used throughout the rest of the thesis when referring to the university. 


\subsection{Literature Review}

\subsubsection{An indoor environment unlike others}

The Oxford English Dictionary defines 'environment' as "that which surrounds and especially the conditions under which any person or object lives, is developed, or operates (Jessop, 2012). Therefore, 'environment' is inclusive of enclosed spaces. These spaces are referred to as 'indoor environments', where most people in developed countries spend more than $80 \%$ of their time (Jenkins, Phillips, Mulberg, \& Hui, 1992; and Höppe, 2002). Offices, restaurants, classrooms, and laboratories are just a few examples of indoor environments. Working in any of these places often exposes people to various health and safety hazards (Hill \& Finster, 2016), and at different levels of severity. For example, cooking activities in restaurants exposes workers to increased levels of carbon dioxide, which if inhaled in high concentrations can adversely affect respiratory functions (Lee, Guo, Li, \& Chan, 2002; and Lee, Li, \& Chan, 2001).

Although the work conducted in laboratories helps further scientific knowledge and societal advancements, labs are often perceived as dangerous learning and research environments (Wu et al., 2007; Langerman, 2009a; Huising \& Silbey, 2011; and Watch, Tolat, \& McNay, 2010). Unlike other indoor environments, working in academic laboratories involves potential exposure to a wide variety of hazards such as chemical, biological, radiological, electrical, mechanical, physical, etc. (Hill Jr., 2007; and Tenkate, McBride, \& Richard, 2001; Langerman, 2009a; and Shariff \& Norazahar, 2012). Therefore, lab personnel should be aware of the hazards risks (actual and/or potential) associated with their work chemicals and biological substances.

\subsubsection{Chemical hazards in the wet lab environment}

In most universities around the world, Chemistry and Biology are two fields offered by science departments. Generally in these fields, chemicals are handled on a regular basis for learning and research purposes (Adane \& Abeje, 2012; Huising \& Silbey, 2011; and Abbas, Zakaria, \& Balkhyour, 2015). The handling mostly occurs in wet lab spaces provided by the academic institution. The chemicals are generally associated with various hazard classes and hazard severity levels (Adane \& Abeje, 2012; Hill Jr., 2010; Gutiérrez, Emery, Whitehead, \& Felknor, 2013; and Walters \& Keith, 2007). In Canada, the Workplace Hazardous Materials Information System (WHMIS) groups chemicals into the following six hazard classes: 1) gases under pressure, 2) flammable and combustible, 3) poisonous and toxic, 4) corrosive, 5) oxidizing, and 6) highly reactive ( Côté et al., 1998; Langerman, 2009b; and Government of Canada, 2011). Some chemicals are associated with multiple hazard classes (Hill Jr., 2010). Each 
chemical is assigned a specific pictogram to help lab personnel identify the chemical's hazard class (Hill Jr., 2010; CCOHS, 2016; and Government of Canada, 2016a). (See Table 1.1 for a summary of each hazard class along with their pictograms.)

Additionally, WHMIS groups chemicals into hazard severity levels by assigning them one of two signal words: warning, which indicates less severe hazards, or danger, used for high-risk chemicals (Hill Jr., 2010; Workplace Safety North, 2015; and Government of Canada, 2016b). Through these signal words, lab personnel are made aware about the level of danger associated with their work substances. This awareness enables them to employ the appropriate measures necessary for the safe handling of their chemicals.

\subsubsection{Biological hazards in the wet lab environment}

Working in wet labs may also involve coming into contact with various biological substances. These include living organisms (i.e. bacteria, fungi, plants, parasite, etc.), non-living organisms (such as viruses, prions, etc.), and biological toxins (Ruffing, 2013; Gutiérrez et al., 2013; McLeod, 2011b; and Scheid, 2015). Similar to chemicals, a degree of hazard and risk level is associated with these substances. This is generally based on the agents' pathogenicity, infectious ability, transmission mode, host range, and availability of preventative measures as well as effective treatment (Public Health Agency of Canada, 2005). In fact, the Centers for Disease Control and Prevention categorizes biological agents into four risk groups (Tenkate, McBride, \& Richard, 2001; and Chosewood \& Wilson, 2009). Depending on their risk groups, biological agents can be safely handled in one of four containment levels (Chosewood \& Wilson, 2009; Furr, 2000; \& Haybaeck et al., 2016).

Due to the wide range of hazards associated with lab chemicals and biological substances, academic wet laboratoris are considered dangerous workplaces (Wu et al., 2007; Langerman, 2009a; Huising \& Silbey, 2011; and Shariff \& Norazahar, 2012). Therefore, safety must be a top priority in these indoor environments (Abbas, Zakaria, \& Balkhyour, 2015). Additionally, it is crucial to ensure that workers are well equipped (i.e. trained, provided with the necessary safety equipment, etc.) to manage (handle, store, dispose of) their lab substances appropriately (Wu et al., 2007). Unsafe management of lab chemicals and/or biological agents may result in undesirable effects on the environment and adversely affect the health and safety of exposed workers.

\subsubsection{Adverse impacts of improper lab practices}

The mismanagement of lab chemicals may adversely impact the lab and/or the outdoor environment (Artdej, 2012 and Shariff \& Norazahar, 2012). For example, chemical fume hoods should be utilized when conducting toxic 
experiments, in order to exhaust any generated toxic aerosols to the outdoors (Berkeley Lab, 2000; \& Creighton University, n.d.). However, when the hoods are either not available or used improperly, the fumes can circulate in the lab and jeopardize the air quality (Berkeley Lab, 2000; \& Chien, Chang, and Lo, 2009). Additionally, unsafe handling of the substances could also increase lab emissions (Reinhardt, Ashbrook, \& Leonard, 1995). Furthermore, properly handling lab chemicals also involves the safe management of the lab's generated wastes, the majority of which are hazardous (Allen, 1983; \&Armour, 2003). Improper disposal or diversion of the wastes may adversely impact the environment (Furr, 2000, p. 434). Specifically, the following consequences may arise: water quality issues that will damage aquatic organisms, polluted atmospheric air, sewer system damage, etc. (US National Research Council, 1995; \& Blackman, 2011). For example, when corrosive, radioactive, flammable, and reactive chemicals are disposed down the drain, they can interfere with the proper operation of sewage treatment facilities, create fire hazards, corrode lab and building plumbing, and generate hazardous gases (US National Research Council, 1995; \& Berkeley, 2013).

Also, if the drainage pipes are old, which is often the case in most universities, the hazardous chemical(s) could leak or escape from the sewer pipes and eventually pollute the university campus ground and/or the outdoor air (Blackman, 2001; \& Berkeley, 2013). In addition to threatening the safety of the lab and natural environment, unsafe management of lab chemicals may result in adverse health and safety effects on workers, students, faculty, and the general public. For example, lab accidents may occur and potentially cause minor or major injuries (e.g. burns, lacerations, eye and finger injuries, damage to respiratory track from chemical inhalation, etc.) and even fatalities. Therefore, it is important to ensure that lab personnel are fully equipped to carry out their activities safely. However, in the past most lab workers were not adequately equipped with the necessary tools to perform their work safely. This led to numerous severe injuries and fatalities among scientists, some of whom were well known.

\subsubsection{Laboratory safety management in the past}

\subsubsection{Lab safety history}

Though great scientific discoveries were made in the past and eventually helped to enhance society, the concept of safety was absent in most of the work that led to them. In fact, in the early 1840s, lab safety was given very little attention (Hill Jr, 2007). Specifically, researchers were ill-equipped to conduct their work safely. As a result, they often handled hazardous substances on open benches instead of in appropriate safety devices (e.g. chemical fume hoods), managed hazardous substances without wearing the necessary personal protective equipment 
(PPE), pipetted hazardous materials by mouth, poured hazardous chemicals down the drain, etc. (Sewell, 1995; Furr, 2000; \& Miller et al., 2012). Furthermore, the labs in the past were "...full of dense fumes and noisome odors" (Hill Jr., 2007).

Also, martyrdom for the sake of science was considered justifiable and acceptable by most researchers at the time (US National Research Council, 2011b). According to an 1890 address made by the well-known scientist August Kekule, to become a chemist in those days, one had to ruin one's health because “....who does not ruin his health by his studies will get nowhere in chemistry" (Hill \& Finster, 2016; \& US National Research Council, 2011b). In fact, a number of well-known scientists suffered severe injuries and fatalities. For instance, in 1843, a bottle holding a very toxic chemical (cacodyl chloride) exploded in the face of Robert Bunsen, the chemist known for inventing the Bunsen burner (Michalovic, 2008). As a result, Bunsen permanently lost his right eye, and almost lost his life after inhaling the toxic vapors from his experiments (Michalovic, 2008). Additionally, the Nobel Prize winner, Marie Curie, known for discovering rubidium and polonium, died due to exposure to her radioactive experimental materials (Bagley, 2013).

As accidents like these continued to take a toll on many scientists, interest in lab safety began to rise within academia (Hill Jr., 2007). Many universities attempted to incorporate safety in their academic curriculum (Lowry, 1978; Hill Jr., 2007; and Ruffing, 2013 ). Additionally, various studies were undertaken and papers written to 1) help workers better understand the safety conditions present within their labs and 2) equip them with tools on how they can address safety issues appropriately.

\subsubsection{Lab safety papers, studies, and surveys}

One of the first papers on lab safety was written in 1910, and titled "Hygiene of the Small Chemical Laboratory" (Ruffing, 2013). It suggested that ventilation should be used to dissipate hazardous fumes and toxic substances out of the lab environment (Keller, 1910; Ruffing, 2013; \& Hill Jr., 2007). Twenty-four years later, another paper brought forth the concept that safety should not just be the responsibility of those in direct contact with the lab substances, but also the concern of those in charge, such as lab supervisors, managers, etc. (Ruffing, 2013). For academic laboratories specifically, a paper on how university labs can properly store their chemicals was published in 1925 (Hill Jr., 2007; \& Ruffing, 2013). Also published in that year was a paper that provided suggestions to teachers about the actions to take in case of potential lab emergencies such as poisonings (Hill Jr., 2007). 
The 1949-1979 survey conducted by Pike and Sulkin (1951) was one of the most comprehensive reviews of unreported infections from lab work in the United States and around the world (Sewell, 1995). The survey showed a total of 3,921 cases of lab-related infections that had occurred and adversely affected various workers in the past. Of these cases, 157 resulted in fatalities, 2,744 led to needle-stick injuries, 1,058 resulted in splashes or spills, and 627 resulted in cuts. The survey analysis showed that $59 \%$ of the 3,921 incidents occurred in research laboratories (Sewell, 1995). Furthermore, in 1984, Kaufman and Curry College conducted a national lab safety survey across the U.S. (Pesta \& Kaufman, 1986). This study involved the participation of 2,019 universities and colleges. The results revealed a total of 2,704 lab injuries (of which 1,171 had to be treated), 178 of which led to fires and 72 resulted in explosions.

In addition to the above lab safety papers and studies, occupational health and safety regulations, standards, and guidelines were also developed in many countries to help enhance safe lab practices (Karapantsios, Boutskou, Touliopoulou, \& Mavros, 2008; and US National Research Council, 2011b).

\subsubsection{Regulations and guidelines for lab substances}

In the province of Ontario, Canada, the safe handling of hazardous chemical and biological substances in laboratories broadly falls under the Occupational Health and Safety Act (OHSA). The OHSA has numerous regulations (e.g. WHMIS, Industrial Establishments, Health Care and Residential Facilities, etc.), which are specific to certain workplace hazards. It came into force in 1979 after the realization that accidents were happening in workplace settings other than factories and industries (Government of Ontario, 2014 \& Ministry of Labour, 2015). The Act operates under the principle of the Internal Responsibility System (IRS), which means that every individual in the organization has a role to play in keeping their work environment safe and healthy (Ministry of Labour, 2015a). However, the OHSA vests ultimate responsibility for safety on employers (e.g. the academic institution, etc.) and/or supervisors (Ministry of Labour, 2015a). Furthermore, it requires that employers and supervisors always exercise due diligence when it comes to workplace safety. In other words, they are to take every reasonable precaution for the protection of their workers (Government of Ontario, 2014). According to sections 66 to 69 of the OSHA, non-compliance may result in an offense and, upon conviction, the individual could become liable to a fine, imprisonment or both (Government of Ontario, 2014). 


\subsubsection{Biological agents: Canadian regulations and guidelines}

All lab activities in Canada that involve the handling, storing, and disposal of biohazardous substances are regulated by the Public Health Agency of Canada (PHAC) (Haybaeck et al., 2016).Standard and guideline documents have been developed by the Agency to outline lab safety requirements. Example of these documents

include: the Canadian Biosafety Standard (CBS), $2^{\text {nd }}$ Edition, 2015; the Canadian Biosafety Handbook (CBH), $2^{\text {nd }}$ Edition; and the Canadian Biosafety Guidelines (Government of Canada, 2015; Public Health Agency of Canada, 2016; Speare, 2015; and Haybaeck et al., 2016).

\subsubsection{Chemical agents: Canadian regulations and guidelines}

Hazardous chemicals on the other hand are governed by WHMIS, a national piece of legislation that became effective in 1988 and is regulated at the provincial level (CCOHS, 2012; Ministry of Labour, 2013 and 2008). WHMIS is designed to ensure that employees are given the appropriate information about their work chemicals and are instructed in the safe handling of those chemicals (Ministry of Labour, 2008; \& Workplace Safety North, 2015). In 2015, WHMIS was modified to incorporate the Globally Harmonized System of Classification and Labelling of Chemicals (GHS), which sets a standard way for classifying and communicating the hazards of chemicals (Hill Jr., 2010; and CCOHS, 2015). The modified WHMIS is now referred to as WHMIS2015(Health Canada, 2015; Staley, 2015; \& Workplace Safety North, 2015).

WHMIS requires that every worker handling or coming into contact with hazardous chemicals is 1) made aware of the hazards and risks associated with the chemicals and 2) adequately trained in the proper management of those chemicals. For workers to be educated in the hazards and risks associated with their lab chemicals, WHMIS requires that all lab chemicals have a supplier or workplace label (Figures 1A and 1B), and that updated Safety Data Sheets (SDSs) be made available for each chemical CCOHS, 2016b). To ensure that workers are aware of the proper way to manage their lab chemicals, WHMIS also requires that all lab personnel or other individuals who will come into contact with any hazardous chemical receive the necessary safety training (CCOHS, 2016a).

In addition to WHMIS, there are guidelines established for proper handling of lab chemicals. For instance, there is the 'Laboratory Health and Safety Guideline' (Diberardinis \& Baum, 2013), a document jointly developed by the Chemical Institute of Canada and the Health and Safety Committee of the Ordre des Chimistres du Quebec. This guideline is intended to help enhance workers' lab practices. The importance of the above regulations and guideline is evidenced by the fact that full compliance by all stakeholders (employers, supervisors, and workers) 
leads to greater productivity and cost savings for the lab, as well as an overall safer lab environment (US National Research Council, 2011b; \& World Health Organization, 2004). However, many have recently questioned the safety conditions in today's academic lab environment.

\subsubsection{The current state in today's academic wet labs}

\subsubsection{Safety conditions in today's university wet labs}

Despite the many safety regulations, guidelines, and additional tools available today, safety continues to be an issue in the wet labs of many universities in the U.S. and around the world. In fact, the manager of a chemical company stated: "We see the product of our colleges and universities as summer employees, temporary employees, and permanent employees. They range from undergraduate through postdoctoral levels. All have one thing in common - no conception of safety" (Kaufman, 1986). Additionally, the safety conditions in university labs are described by the president of Laboratory Safety Institute (Kaufman, 1978, and 1986) as "shameful, horrifying, and notoriously bad". During the Panel Discussion on Laboratory Safety Culture at the $46^{\text {th }}$ Midwest and $39^{\text {th }}$ Great Lakes Joint Regional Meeting, organized by the American Chemical Society in 2011, it was commonly agreed that "safety must be of utmost importance in our teaching and research labs" (Backus et al., 2012). This suggests that safety is currently not given the priority it deserves in today's university wet labs. This is supported by the many accidents that have recently plagued various academic labs in the U.S. and around the world (Wu, Liu, \& Lu, 2007b; and Nara, 2016).

The news headlines collected by the American Chemical Society (ACS) in the past few years have shown that lab accidents within universities occur frequently (Reeger, 2012). In fact, according to the president of Laboratory Safety Institute, these accidents are 10 to 50 times more common in academic settings than in chemical industries (Vergano \& Korte, 2011; Eldridge, 2012; Kaufman, 1978; and Benderly, 2009). Furthermore, information gathered from the U.S. Chemical Safety Board (2010) (CSB) showed that, since 2001, at least 120 accidents have occurred in the wet labs of various universities (Vergano \& Korte, 2011). See Table 1.2 for a list of some of these accidents. For an additional list of academic laboratory accidents, refer to the following websites:

1). www.labsafetyintitute.org/MemorialWall.html,

2). www.ehs.ucsb.edu/labsafety/laboratory-accidents,

3).www.aiha.org/get-involved/VolunteerGroups/LabHSCommittee/Incident\%20Pages/Lab-Safety-

Explosions-Incidents---Chemistry.aspx.) 
These accidents caused damage to lab facilities as well as resulted in adverse health and safety effects on exposed individuals (Hoff, 2003; \& Kemsley, 2012). It is worth noting that some of these accidents have also led to lawsuits against universities and academic supervisors (Hoff, 2003). One recent lawsuit occurred after the 2008 fatal chemistry lab accident at the University of California Lost Angeles (Benderly, 2014). Reports showed that after the fatality, the victim's professor faced four felony charges for violating California's occupational safety laws (Cal/OSHA) (Benderly, 2014). Some of the violations include lack of appropriate safety training, failure to issue PPE to victim, etc. (Skvorc \& Wilson, 2015). Some in the literature say that these accidents are a result of carelessness or ignorance, because various significant safety factors (e.g. provision of PPE, adequate training, etc.) that should have been addressed by both management and lab workers were not (Hoff, 2003).

From these accidents, it is evident that safety continues to be a significant issue in the wet laboratories of today's universities. In fact, after reviewing more than 100 lab accidents, Langerman (2009a), the founder of Advance Chemical Safety Inc. and a regular columnist for the Journal of Chemical Health and Safety, concluded that today's academic labs are not safe environments for studying and/or carrying out research. Additionally, widespread concerns have been raised about the current state of safety culture within the labs (American Chemical Society, 2012; Langerman, 2011; Jia, 2016; Eldridge, 2012; \& US National Research Council, 2014).

\subsubsection{Lab safety Culture}

The term 'safety culture' was coined by the International Atomic Energy agency after investigating the 1986 Chernobyl disaster (Gadd \& Collins, 2002; McGarry et al., 2013; Mearns \& Flin, 1999; Lau \& Ng, 2013; and US National Research Council, 2014). Safety culture measures the perception, attitudes, beliefs, norms, and behaviours adopted towards safety for an extended period of time (Eldridge, 2012;WEIL, 2012, p. 136; \& Choudhry, Fang, \& Mohamed, 2007). In their report entitled "Creating Safety Cultures in Academic Institutions: a Report of the Safety Culture Task Force ACS Committee on Chemical Safety", the American Chemical Society's Committee on Chemical Safety (ACSCCS) (2012, p. 10; and Hill \& Finster, 2013) stated that "....the attention and priority that an institution gives to safety will reveal the level of importance placed on it and ultimately determine the strength of its safety culture" (Hill \& Finster, 2013; \& American Chemical Society, 2012, p. 14).

Having a strong safety culture enables compliance to lab safety rules and conformance with best practices (American Chemical Society, 2012a). A strong safety culture is influenced by various factors and elements. Gadd and Collins (2002) argue that it is mainly characterised by the commitment of senior management to safety, realistic 
and flexible procedures established for the safe handling of both well and ill-defined hazards, continuous organizational learning through feedback systems, monitoring, and a shared care and concern for the hazards in the lab. The critical factor is leadership, as this has the most impact and influence on the other elements (Hill \& Finster, 2013, p. 28; \& American Chemical Society, 2012). In support of this, the U.S. National Research Council stated that "The ultimate responsibility... for encouraging a culture of safety rests with the head of the organization (or institution) and its operating units. Leadership by those in charge ensures an effective safety program is embraced by all” (American Chemical Society, 2012; \& US National Research Council, 2011).

Additionally, a strong safety culture is easily built when the workplace has a well-functioning top-down management system, in which safety responsibilities are shared by all, and safety efforts (i.e. safety programs, etc.) are uniform among all stakeholders i.e. supervisors and workers (Gutiérrez et al., 2013). Nonetheless, a strong culture of safety is also facilitated when safety is actively lived out and viewed as an essential part of the work environment by both managerial and non-managerial individuals (American Chemical Society, 2012). This is because "ensuring a safe lab environment is the combined responsibility of management, Environmental Health and Safety personnel, and laboratory workers i.e. students” (US National Research Council , 2011).

\subsection{Safety culture within academia: a need for improvement}

Due to the high number of recent accidents (Eldridge,2012; \& Environmental Health \& Safety, 2014), today's academic wet labs have gained the reputation of lacking a strong and positive safety culture (US National Research Council, 2014). The need to improve the safety culture of university-wet labs was further evidenced by the findings from an international study and various smaller studies (McLeod, 2011a; Huising \& Silbey, 2011a; Van Noorden, 2013;Wargniez, Oleas, \& Yamaguchi, 2012; and Stanford University, 2014).

In 2012, a comprehensive laboratory safety culture survey was jointly conducted by the University of California (UC) Center for Laboratory Safety, BioRaft, and Nature Publishing Group (Schröder et al., 2016; \& Wayne, 2016). The survey was administered internationally to researchers from academia (which had the highest number of participants), industries and government laboratories (Schröder et al., 2016). Findings revealed the following: 1) researchers from academia, compared to industry, were less compliant with wearing required lab coats and eye protection (Van Noorden, 2013; \& Mohammed, Korsa, Soltan, \& Roshdy, 2015 and Kemsley, 2013); 2) in

contrast to industry researchers, about $12 \%$ of academic researchers do not assess for any risks before proceeding with experiment(s) (Kemsley, 201 and Schröder et al., 2016); 3) 64\% of academic researchers reported that on 
multiple occasions, people work alone in their labs (Watson, 2013); and 4) while 90\% of all survey participants said that they are aware of and understand the minimum training requirements for their lab duties, $40 \%$ revealed they did not receive any safety training on their specific lab agents (Mohammed et al., 2015).

Furthermore, a study conducted by Stanford University (2014) showed that safety is not a high priority for $30 \%$ of the university's lab personnel. Many of the study participants commented that lab safety is not well communicated at the university (Stanford University, 2014). After being inspected by the Environmental Protection Agency (EPA), the lab safety management at Eastern University was found lacking a clear hierarchical system for compliance with environmental laws, a systematic approach to environmental management, a clear delineation of roles and responsibilities, and a safety accountability system for lab personnel (Huising \& Silbey, 2011a). Lastly, the unannounced inspection of the labs at New Jersey City University showed the following safety violations: improper chemical storage practices, improper handling and storage of hazardous wastes, a lack of chemical inventory listing, and failure to ensure lab personnel have hazardous waste management training (Wargniez et al., 2012a).

The results from the aforementioned studies and others (Karapantsios et al., 2008; Ferjencik \& Jalovy, 2010; Artdej, 2012; Sedghpour, Sabbaghan, \& Sataei, 2013; Gallion, Samide, \& Wilson, 2015; and Wyllie, Lee, MorrisBenavides, \& Matos, 2016) have led safety professionals to conclude that academic researchers run their labs with less attention paid to safety compared to industrial researchers (Kemsley, 2012). Unlike universities, industry laboratories operate under a clear hierarchy of power and with fewer inexperienced workers; they also hold management accountable when safety standards are not upheld or regulatory requirements are breached (Van Noorden, 2011). However, since there is currently no literature on the manner in which safety is practiced and managed within Canadian university laboratories, this conclusion cannot be generalized to include Canadian researchers. Nonetheless, as many government agencies, professional societies, and academics from the US and other countries have resolved to re-examine the state of safety in their laboratories (Rugani, \& Dickson, 2014), it would be beneficial to also examine the current state of safety within Canadian university laboratories. This will help ascertain the level of risk (actual or potential) present in the lab environments. Therefore, the current study examined the state of safety that exist within the wet laboratories of a medium-sized Canadian university. 


\subsection{Research Study setting}

\subsubsection{Lab safety initiatives at University $X$}

The Canadian university being examined in this study, University X, houses over 100 wet laboratories used by various study fields (E. Ambroise, V. Phelan, personal communication, September, 2015). University X has established an Environmental Health and Safety Policy, which outlines safety roles and responsibilities for all members of the university (Integrated Risk Management, 2011). It has also established a Workplace Inspection Program, which requires that all university premises be annually inspected, as per section 9 of the OHSA. Additionally, the university has created a department, the Integrated Risk Management, to assist faculty, staff, and students in fulfilling their OHSA responsibilities. To help facilitate lab personnel's compliance with safety requirements, this department has developed numerous programs and training modules (e.g. laboratory safety signage, WHMIS training, Biosafety program, Risk assessment management, etc.) (Integrated Risk Management, n.d.). However, every semester a few lab incidents occur, including an acid burn reported in the fall of 2016 (S. McFadden, personal communication, April 7, 2017). Therefore, although the above initiatives are well-intentioned, it is uncertain how well enforced they are to maintain safety within the University's lab environments. Additionally, findings from historical safety reports raise numerous causes for concern about the labs 'state of safety' and the overall strength of the Internal Responsibility System within the University.

\subsubsection{Past safety reports}

Verbal communication and email correspondences with staff from the University's Environmental Health and Safety (EHS) team, revealed the following safety reports: 1) a walkthrough carried out by University X's EHS team in February 2012 for its chemistry and biology laboratories.; 2) an October 2012 safety audit conducted by an external company in response to safety concerns previously raised by a city of Toronto Fire Inspector; and 3) a 2013-2014 campus-wide safety inspection conducted by the University's EHS team, to address the deficiencies identified by the October 2012 safety audit. These reports revealed numerous safety issues within the labs. A select list of the issues include, but not limited to:

- Excessive volumes of flammable liquids not stored in approved safety cabinets,

- Many Safety Data Sheets not up-to-date,

- Improper segregation of chemicals,

- Some gas cylinders not tagged to indicate cylinder status, 
- Incompatible chemicals stored together,

- $\quad$ Fume hoods used for lab waste storage,

- $\quad$ Some chemical containers not properly labeled and are corroding,

- Many labs without chemical spill kit/clean up material, fire extinguishers, PPE, and eyewash stations, etc.

\subsubsection{A need for further safety examination}

Given these safety issues, as well as the increased number of accidents that have been recently reported from many universities around the world, the current state of safety within the labs at this medium-sized Canadian university is not known. Specifically, it is unknown if the deficiencies identified by the safety reports have been addressed to ensure that the labs are in safe conditions. Therefore, it was deemed necessary to examine the state of safety that currently exists within University X's chemistry and biology wet laboratories. The goal was to ascertain if lab personnel face any health and safety risks when in their work environment(s).

\subsection{Overall research objectives and methods}

This research has the following three major objectives:

1) Obtain a better understanding of the substances present within University X's wet laboratories. This data was obtained by inventorying the chemicals and biological agents present within the labs that participated in the research.

a). Since risk perception influences lab practices and management (Furr, 2000), part of this objective was to identify how workers perceive the level of risk associated with their lab work. This data was obtained via an online survey.

2) Identify any hazardous and risky conditions present within the labs. The data for this objective were collected by inspecting all the labs that participated in the research. Also, some of the inspection findings from this research were compared to the findings from the historical safety reports previously mentioned. The purpose of this comparison was to identify if there are any reoccurring safety issues in the labs.

3) Identify the overall culture of safety that exists within the labs at University X. This was achieved by administering an online safety culture survey to all study participants. The survey questions were mostly taken from the 2012 international lab safety culture survey.

It should be noted that some questions from the survey were utilized to obtain data for objectives \#1 and 2. Also, since the ultimate objective of this research was to get a descriptive understanding of how lab safety may be 
perceived and managed at University X, all collected data were descriptively analyzed. However, to compare the difference in risk perception between genders and field studies, an inferential analysis was further conducted on the risk perception data collected. 


\begin{tabular}{|c|c|c|}
\hline $\begin{array}{c}\text { Chemical } \\
\text { hazard class }\end{array}$ & Hazard class description & Hazard class symbol \\
\hline Compressed Gas & $\begin{array}{l}\text { Dangerous if broken, heated, or leaking. } \\
\text { The additional danger may come from the gas property i.e. flammable etc. } \\
\text { Common examples: Carbon-dioxide, Nitrogen, Ethylene-Oxide, Propane, Oxygen, etc. }\end{array}$ & \\
\hline $\begin{array}{l}\text { Flammable\& } \\
\text { Combustible } \\
\text { Materials }\end{array}$ & $\begin{array}{l}\text { Can easily ignite (burn or catch fire): some ignite at normal temperature; others when } \\
\text { heated and at temperature above normal; and others ignite when in contact with water } \\
\text { or air } \\
\text { Typical examples: Acetone, ethanol, Toluene, butane, Stoddard solvent, etc. }\end{array}$ & \\
\hline Oxidizing Material & $\begin{array}{l}\text { May cause or intensify fire, or cause an explosion } \\
\text { Some (organic peroxides) will burn as well as provide the oxygen needed to cause a } \\
\text { fire } \\
\text { Typical examples: ozone, nitric acid, potassium permanganate, sodium chlorite, } \\
\text { perchloric acid solution, etc. }\end{array}$ & \\
\hline $\begin{array}{l}\text { Poisonous\& } \\
\text { Infectious }\end{array}$ & $\begin{array}{l}\text { Harmful to life and health: the effects can be immediate and severe, temporary, or } \\
\text { disease causing. } \\
\text { Typical examples: Carbon monoxide, Sulphuric acid, benzene, mercury, bio-hazardous } \\
\text { agents such as salmonella, hepatitis B, HIV virus, etc. }\end{array}$ & \\
\hline Corrosive Material & $\begin{array}{l}\text { Can cause severe burns to skin or other human tissue (i.e. eyes, and lungs), as well as } \\
\text { can attack clothes, metals, and other materials. } \\
\text { They may also cause fires when in contact with organic matter. } \\
\text { Typical examples: Sulphuric and nitric acids, ammonium hydroxide, chlorine, nitrogen } \\
\text { dioxide, etc. }\end{array}$ & \\
\hline Dangerously Reactive & $\begin{array}{l}\text { Known for being 'unstable' because they can: } \\
\text { 1) strongly and readily react with water to make toxic gas, } \\
\text { 2) react with themselves when receives a shock or if temperature or pressure increases, } \\
\text { 3) join to themselves, break down or lose excess water to render it very dense. } \\
\text { Common examples: picric acid, anhydrous aluminum chloride, ethyl acrylate }\end{array}$ & \\
\hline
\end{tabular}

(Government of Canada, 2011; Hill Jr., 2010; Government of Canada, 2016a; and Google Images) 


\section{Table 1.2: Accidents cases within the wet labs of universities in the U.S. and around the world}

\begin{tabular}{|c|c|c|c|}
\hline $\begin{array}{l}\text { Accident } \\
\text { year }\end{array}$ & Accident location & About the accident & Accident Outcome(s) \\
\hline 2001 (July) & $\begin{array}{l}\text { The university of California, Irvin } \\
\text { (U.S.) }\end{array}$ & $\begin{array}{l}\text { A walk-in chemical fume hood holding a defective } \\
\text { distillation unit used for purifying benzene released vapor } \\
\text { streams of benzene } \\
\text { At the time of accident, a solvent was improperly stored in } \\
\text { the fume hood, resulting in a hood fire }\end{array}$ & $\begin{array}{l}\text { Burns on student's neck, legs, and arm Extensive damage to two } \\
\text { labs and an instrumentation room. } \\
\text { Smoke and water damage were also observed in the fire sprinkler } \\
\text { system of the building, }\end{array}$ \\
\hline 2002 (January) & $\begin{array}{l}\text { University of California, Santa } \\
\text { Cruz (U.S.) }\end{array}$ & $\begin{array}{l}\text { Fire broke out from a lab bench due to: } \\
\begin{array}{l}\text { - } \\
\text { - }\end{array} \quad \text { a flammable on unattended, or } \\
\text { disposed of. }\end{array}$ & $\begin{array}{l}\text { Two labs completely destroyed } \\
\quad \text { Research work lost }\end{array}$ \\
\hline 2005 (October) & $\begin{array}{l}\text { Cleveland State University } \\
\text { (U.S.) }\end{array}$ & $\begin{array}{l}\text { Biology professor electrocuted in science lab after: } \\
\text { Inserting the plug of a faulty lab equipment } \\
\text { into an outlet }\end{array}$ & Fatality of Professor \\
\hline 2006 (March) & $\begin{array}{l}\text { University of Haute Alsace } \\
\text { (France) }\end{array}$ & $\begin{array}{l}\text { Explosion in research lab caused by ethylene compressed } \\
\text { gas that reached explosive limits due to: } \\
\quad \quad \text { unsafe experimental device i.e. substandard } \\
\text { rubber tubes, } \\
\text { Open and unattended compressed gas cylinder valve }\end{array}$ & $\begin{array}{ll}> & \text { Fatality of a professor } \\
& \text { Nearby students severely injured } \\
& \text { Damages to lab building }\end{array}$ \\
\hline 2006 (February) & Texas A\&M University (U.S) & $\begin{array}{l}\text { Failure and rupture of a compressed Nitrogen gas cylinder } \\
\text { due to: } \\
\text { Pressure valves removal from defective } \\
\text { cylinder } \\
\text { Over pressurization of chemical inside the cylinder }\end{array}$ & $\begin{array}{ll}> & \text { Damaged lab floor } \\
& \text { Fume hood destroyed- resulting in significant damages to } \\
\text { pipelines } & \\
>\quad \text { Water contamination as a result of broken waterlines } \\
\quad \text { Chemical spilled- resulting in contaminations }\end{array}$ \\
\hline 2008 (December) & $\begin{array}{l}\text { University of California Lost } \\
\text { Angeles (U.S.) }\end{array}$ & $\begin{array}{l}\text { Lab fire caused by: } \\
\qquad \quad \text { improper handling of highly toxic chemical, } \\
\text { - lack of wearing PPE, } \\
\text { - lack of formal lab safety training provision, } \\
\quad \text { chemical }\end{array}$ & Fatality of student \\
\hline 2009 (September) & University of Chicago (U.S.) & $\begin{array}{l}\text { Faculty researcher infected with Yersinia Pestis due to: } \\
\text { inconsistency in his PPE (i.e. gloves) usage } \\
\text { during lab work, } \\
\text { - lack of biosafety training attendance etc. }\end{array}$ & Fatality of the researcher \\
\hline 2010 (January) & Texas Tech university (U.S.) & $\begin{array}{l}\text { Lab explosion due to: } \\
\text { No PPE usage during hazardous } \\
\text { experimentation, } \\
\text { lack of formal lab safety training as well as } \\
\text { familiarity with experimental toxic chemical, } \\
\text { lack of lab safety manual or protocol to advice } \\
\text { on the proper use of experimental chemical, } \\
\text { etc. }\end{array}$ & $\begin{array}{l}>\quad \text { Graduate student lost three fingers; } \\
>\quad \text { obtained burnt hands, and injured eyes }\end{array}$ \\
\hline 2011 (April) & Yale university (U.S.) & $\begin{array}{l}\text { Student's hair entangled in a lab lathe machine due to: } \\
\text { - Student working alone in the lab machine shop } \\
\text { - Student working with untied long hair } \\
\text { Student working without supervision or other } \\
\text { lab personnel around }\end{array}$ & $>\quad$ Fatality of the student \\
\hline 2015 (April) & $\begin{array}{l}\text { University of Mining and } \\
\text { Technology (China) }\end{array}$ & Gas explosion in a chemistry lab & $\begin{array}{ll}> & \text { One fatality } \\
> & \text { Four students with severe injuries }\end{array}$ \\
\hline $\begin{array}{l}2016 \text { (September } \\
21 \text { ) }\end{array}$ & Donghua university (China) & $\begin{array}{l}\text { A lab explosion occurred while three postgraduate students } \\
\text { were conducting lab experiments. } \\
\text { Investigation results not yet released }\end{array}$ & $\begin{array}{ll} & \text { On student suffered minor } \\
& \text { Two students had eyes and face burns, went to hospital }\end{array}$ \\
\hline $\begin{array}{l}2016 \text { (December } \\
\left.18^{\text {th }}\right)\end{array}$ & $\begin{array}{l}\text { Chemical, Tsinghua University } \\
\text { (China) }\end{array}$ & $\begin{array}{l}\text { Hydrogen compressed gas explosionInvestigation results } \\
\text { still underway }\end{array}$ & Fatality of student \\
\hline 2016 (March) & $\begin{array}{l}\text { University of Hawaii, Mona } \\
\text { (U.S.) }\end{array}$ & $\begin{array}{l}\text { Post-Doc working alone with a mixture of Carbon Dioxide, } \\
\text { hydrogen, and oxygen gasses when explosion occurred }\end{array}$ & $\begin{array}{ll} & \text { An arm lost } \\
& \text { Severe other injuries }\end{array}$ \\
\hline
\end{tabular}

(Cotter \& Whitherspoon, n.d.; Gorey, 2005;The SCOTSMAN, 2013; Dong, 2016; Van Noorden 2011; Cyranoski, 2015; Benderly, 2016; Benderly, 2016b; Jia, 2016; Dong, 2016; and Meiping, 2016) 
Figure 1A. Example of a WHMIS supplier label

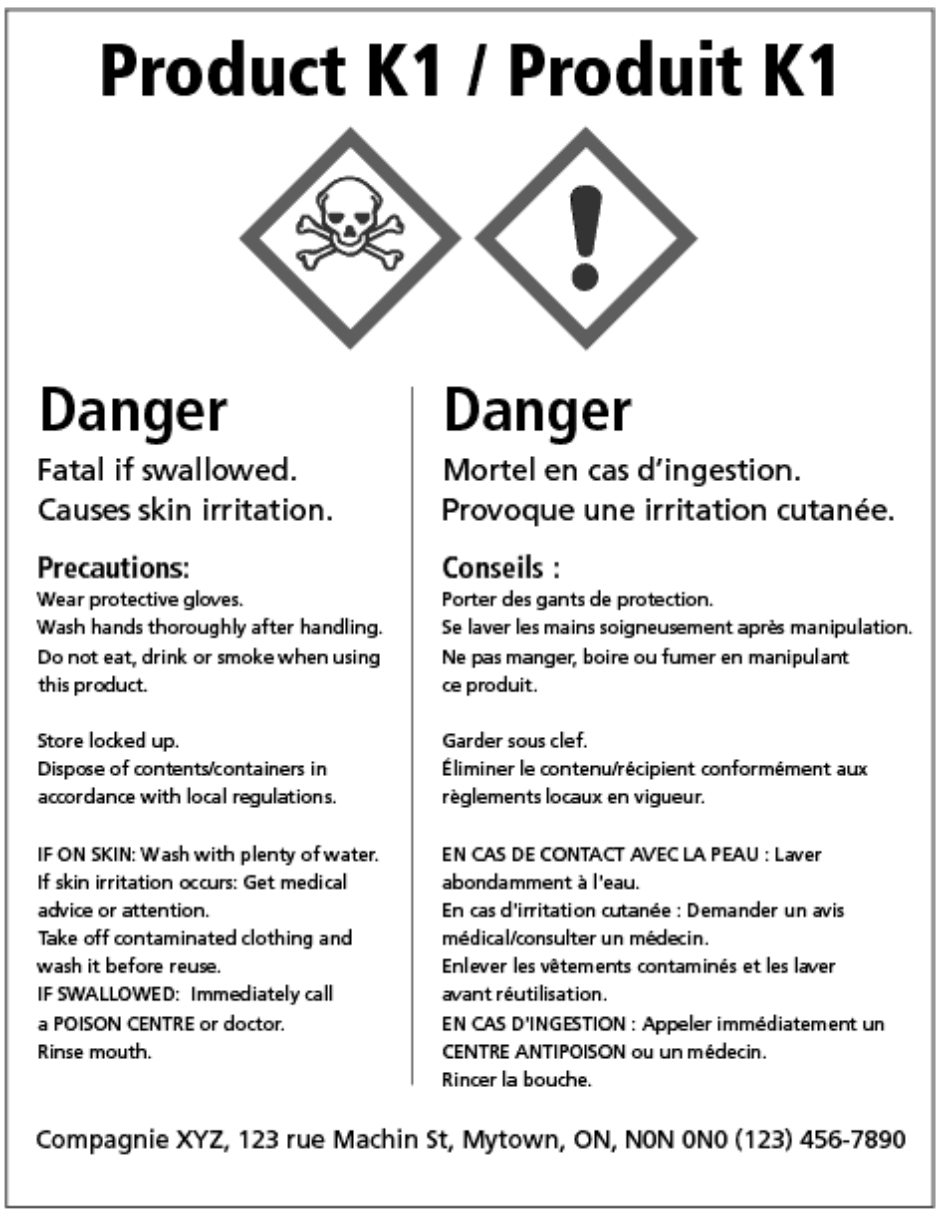

Figure 1B. Example of a WHMIS workplace label

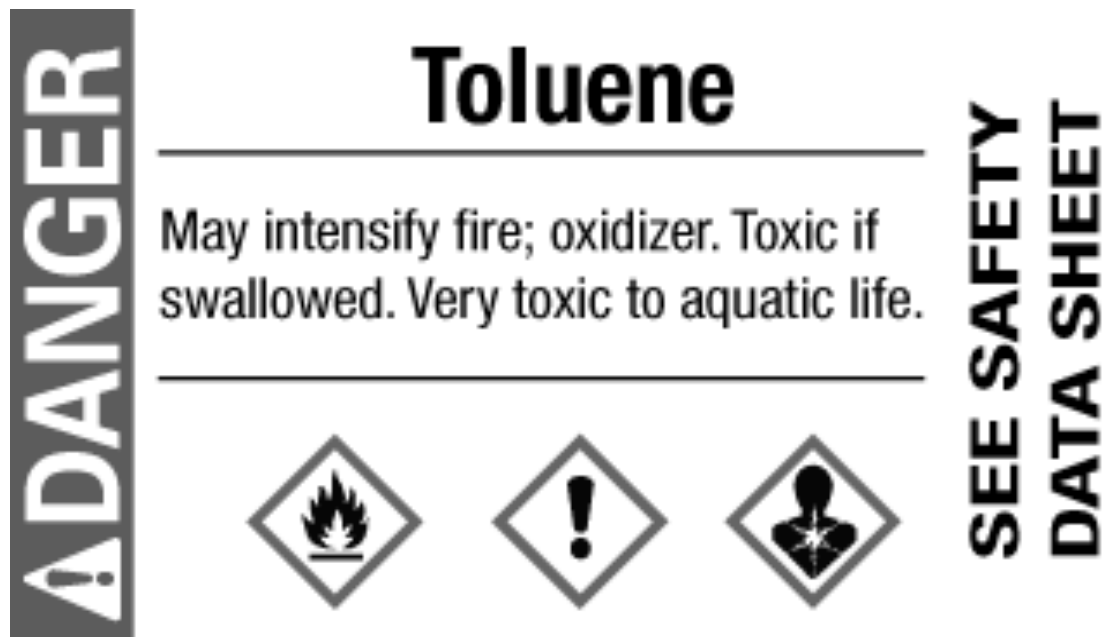




\section{Chapter 2- Academic lab chemical management and workers' risk perception}

\section{Introduction}

Most academic laboratories use chemicals to a certain extent (Karapantsios et al., 2008). Many of these chemicals have inherent hazardous properties (i.e. toxicity, flammability, explosiveness, carcinogenicity, etc.) (Furr, 2000; and Adane \& Abeje, 2012). They can also become hazardous because of accidental mixtures i.e. unsafe reactions (Karapantsios et al., 2008). Therefore, it is essential that lab personnel are made aware of the hazard(s) associated with the chemicals they handle (Karapantsios et al., 2008).

Some of the mandated ways workers can become aware of the hazard(s) associated with their lab chemicals include proper labels, Safety Data Sheets (defined in the Glossary of Terms), training, etc. (Karapantsios et al., 2008). With this awareness, an accurate perception of the risk(s) involved with their lab work can be obtained. As a result of this accurate risk perception, lab personnel holding supervisory roles may become prompted to develop appropriate control measures necessary for keeping their workers and lab environments safe. Lab workers themselves may also become prompted to handle their chemicals safely. This may help minimize or prevent the occurrence of accidents in their lab environments.

Recently, there has been an increasing number of accidents in the wet laboratory of many universities in the U.S. and globally. For instance, in 2008, a chemistry lab at the University of California Los Angeles experienced a fire that led to the death of a research assistant (Van Noorden, 2011). Investigation reports revealed that the tragedy was due to lack of training and improper handling of a highly hazardous chemical (Allen, 2014). In 2015, a lab at Tsinghua University in Beijing experienced a fire that led to the death of a post-doctoral student and severely injured others nearby (Cyranoski, 2015). Investigation reports revealed that the fire was due to an explosion of a hydrogencompressed gas with which the victim was working (Cyranoski, 2015). It is unclear whether the explosion occurred because the gas cylinder had quality or storage issues (Jing, 2015). More recently, in March 2016, a lab explosion at the University of Hawaii caused a post-doctoral researcher to lose an arm (Stoye, 2016). Reports showed the victim used an inappropriate pressure gauge, which was deemed likely to have caused the explosion. Reports also revealed that the pressure gauge was not designed for use in a system that contained a mixture of flammable gas (i.e. hydrogen gas) (Blaszczak-Boxe, 2016). 
These accidents, including others (as per Table 1.2 from Introduction chapter), suggest that university workers may lack adequate awareness about the hazardous nature of their lab chemicals. If they were aware, the necessary precautions would have been taken to at least reduce the likelihood of the accidents. As a result, many safety professionals have concluded that today's academic labs are unsafe and risky environments for learning and conducting research (Langerman, 2009a; Benderly, 2010; and Wyllie et al., 2016 ). Therefore, it is necessary for universities equipped with wet laboratories to ensure that their lab workers are made fully aware of the hazards and risks associated with their work substances.

Similar to other countries, Canada has numerous universities equipped with wet laboratory facilities, where thousands of students are routinely exposed to a wide range of chemicals. However, since no information regarding the safety of Canadian university labs is available, this research investigated the safety conditions of the chemicals present in the wet laboratories of a medium-sized Canadian University (referred to as University X). The goals of this study were: 1) to get a better understanding about the substances present within the University's wet laboratories, and 2) identify if workers and students have an accurate perception of the risk(s) associated with their lab work.

\subsection{Chapter 2 objectives}

The following four objectives were examined to achieve the purposes of this study:

1) identify the chemicals and biological agents present within University $X$ 's teaching and research wet laboratories

2) identify the agents' hazard class and hazard level;

3) identify the labeling and storage conditions of the agents;

4) identify lab personnel's risk perception about their work.

a. Also, determine if there are differences in the risk perception between gender or study fields (chemistry, biology, etc.)

\subsection{Materials and methods}

\subsubsection{Recruitment}

Before starting this study, ethics approval was obtained from University X's Research Ethics Board. Subject and lab recruitment was performed via emails, in person, and by posting of flyers within several buildings of the University. The participation criterion for the labs was: any chemistry and/or biology wet laboratory from the 
University that is currently and actively utilized for research and/or teaching. The subject inclusion criterion was: any member of University X (students, faculty, and staff) currently engaged in wet lab activities at any of the University's wet laboratories.

\subsubsection{Chemical inventory}

To identify the chemicals present within the University's labs, inventories were performed by members of the research team in all participating research and teaching laboratories. A checklist (Appendix A and B), made up of 16 questions, was utilized to carry out the inventories. This was conducted by 1) observing the labs' shelves, floors, cabinets, cupboards, chemical fume hoods, biosafety cabinets, etc., to locate any chemical container; and 2) subsequently using the checklist questions to gather information about the chemical stored inside the container. Specifically, the information gathered included the name and, labelling and storage condition of the chemical.

\subsubsection{Inventory of biological agents}

The biological agents were inventoried via an online survey questionnaire, in which supervisors and technicians were asked to list the three biological agents most frequently handled in their labs. The reason for performing the inventory this way was to avoid any potential interference with the agents' integrity while in their storage environment, which is generally sensitive (i.e. refrigerators).

\subsubsection{Hazard level of the chemicals and biological agents}

Safety Data Sheets (SDSs) retrieved from various online resources (i.e. Fisher, Sigma-Aldrich, etc.) were used to obtain the hazard information of each inventoried chemical. Each chemical's signal word (warning or danger) was identified. The signal words revealed the hazard level associated with the inventoried chemicals. According to the Workplace Hazardous Materials and Information System (WHMIS) (Government of Canada, 2016), warning is used for less severe hazards, while danger is used for high-risk hazards. With this data, the principal investigator enumerated the amount of chemicals associated with both hazard levels (danger and warning).

For the biological agents, various internet resources (i.e. e-books, official websites, journals, etc.) were utilized to identify the agents' risk group, which indicates the agents' likelihood of causing disease in humans, animals, etc. (Virginia University, n.d. ; and Tenkate, McBride, \& Richard, 2001).

\subsubsection{Labeling and storage of the lab substances}

To understand whether or not the chemicals are properly managed, the labeling and storage conditions of each inventoried chemical were examined. The inventory checklist questions that focused on labeling and storage 
were utilized to obtain this data. Examples of questions include: is the chemical container clearly labeled? is storage area clean and uncluttered? does the cylinder (which contains compressed gases) have a legible status tag? etc. (see Appendix A and B for the entire checklist).

\subsubsection{Risk perception of lab workers}

To identify lab personnel's risk perception of their work, all study participants were asked via an online survey to make a qualitative assessment of what they believed is the risk level of the work conducted in their labs. The response options included "very high risk," "high risk," "moderate risk," "very low risk," and "low risk." Subsequently, responses were compared to the results of the inventoried chemicals. This comparison was to see if the risk level identified by lab workers correlates with the hazard(s) associated with the inventoried chemicals.

To identify if risk is perceived differently between genders (female vs male) and/or programs (i.e. chemistry, biology, etc.), the Chi-square test of independence using TIBCO Spotfire S+ was conducted.

All other data for this chapter were descriptively analyzed and depicted in tables and graphs.

\subsection{Results}

\subsubsection{Study participation rate}

Through the various recruitment methods, 34 chemistry and biology wet laboratories were approached. Out of these, 21 labs consented to be inventoried ( $\mathrm{n}=10$ teaching labs, $\mathrm{n}=11$ research labs). Thus, a lab participation rate of $62 \%(21 / 34)$ was obtained. Of the participating research labs $(n=11)$, nine and two came from biology and chemistry programs, respectively. Regarding the teaching labs $(n=10)$, six and four were from biology and chemistry programs, respectively.

Out of approximately 159 lab personnel approached, a total of 61 consented to participate in the online survey. However, 5 participants declined to answer the risk perception question, leaving a final response rate of $35 \%$ (56/159). Of the 56 participants, 47 came from chemistry and biology programs, while 9 were from other study fields e.g. Engineering, etc. Since a majority of the participants came from Chemistry and Biology, the author only focused on responses from these programs. Among the 56 participants, 14 indicated they hold supervisory lab roles (i.e. Principal Investigators, technicians, supervisors, etc.).

\subsubsection{Inventories: Chemicals within the University's laboratories}

The chemical inventories revealed that there are at least 1,128 chemicals present within the 21 wet labs examined in this study. The SDSs information showed that the chemicals are associated with at least one WHMIS 
hazard class, though many were found associated with multiple classes (Table 2.1 ). Also, $77 \%$ of the chemicals were associated with either the 'warning' (29\%) or 'danger' (48\%) signal words (Figure 2.1). The remaining (23\%) inventoried chemicals lacked a WHMIS signal word.

\subsubsection{Inventories: labeling and storage condition of the University's lab chemicals}

\subsubsection{Labeling conditions of the lab chemicals}

Among the chemicals that lacked a signal word, 9\% (over 100) were unlabeled. Even among the labeled chemicals, $40 \%$ (approximately 450 chemicals) had their label content only include the chemicals' name while excluding relevant and mandatory information such as hazard information, safe handling procedures, and reference to safety data sheets. When the SDSs of these ( 450) improperly labeled chemicals were looked up online, the findings revealed that 167 (37\%) of them were associated with the WHMIS signal word 'Danger'.

Additionally, in labs that use compressed gases, $64 \%$ of the compressed gas cylinders were missing a status tag, a label that tells lab personnel whether or not the cylinder is empty, full, or in use.

\subsubsection{Storage conditions of the lab chemicals}

Of the inventoried chemicals, $31 \%$ were stored in alphabetical order, instead of according to their hazard classes or within their compatibility group. On more than 100 occasions, chemicals were found to be incompatibly stored with one another. For example, on 15 occasions acids were found stored in flammable cabinets; on 98 instances, flammables were found stored with incompatible hazard classes such as corrosives, oxidizers, and bases. See Table 2.2 for a few specific examples.

Furthermore, on multiple occasions, fume hoods were utilized for storing chemicals, hazardous wastes, and other lab materials (Figure 2.2). Moreover, many storage areas were found in cluttered (Figure 2.3) and unclean conditions (Figure 2.4), mainly due to rusty and corroded chemical containers. Ironically, some of the labs that had chemicals stored in cluttered conditions had flammable and acid storage cabinets containing only a handful chemicals (Figure 2.5).

\subsubsection{Online survey: Biological agents within examined labs}

Approximately 55\% of the survey participants reported that bacteria are commonly handled in their labs. However, since the biological agents' list provided were generic (i.e. no speciation of agents), it was impossible to identify their risk group. Therefore, no further data could be obtained and analyzed for these agents. 


\subsubsection{Online survey: Lab risk perception among examined lab personnel}

While $48 \%$ of the inventoried chemicals were assigned the 'danger' signal word, only $9 \%$ of the survey participants, mostly from chemistry, indicated "high risk" when asked to express their belief about the risk level associated with their lab work. However, $43 \%(n=26 / 56)$ of the biology respondents indicated "very low to low risk". Although no statistical significance was found with respect to the perception of lab work risk between genders ( $\mathrm{p}=0.31$ ), the Chi-square test results showed that there is a statistically significant difference in the perception of risk by the department $(\mathrm{p}=0.02)$. Specifically, Chemistry survey participants had a higher risk perception than biology participants.

\subsection{Discussion}

The overall purpose of this study was to obtain a better understanding of the chemicals handled within the wet laboratories of a medium-sized Canadian academic institution, University X. The findings indicate that the labs examined have a wide variety of hazardous chemicals. Additionally, similar to other universities (Abbas, Zakaria, \& Balkhyour, 2015;Wargniez, Oleas, \& Yamaguchi, 2012; and Stroud, Stallings, \& Korbusieski, 2007), and to the findings from the University's past safety reports, the labeling and storage condition of many of the chemicals are not in compliance with legal requirements and lab best practices.

For example, many of the inventoried chemicals lacked a WHMIS supplier or workplace label. In fact, this contributed to the difficulty in obtaining hazard information for almost 100 of the inventoried chemicals. Further concerns arose when examining the hazardous information of those chemicals that had a label. The issue was that many $(40 \%)$ of those chemicals had missing essential information such as hazard information, reference to SDS, safe handling procedures, etc. This is a violation of WHMIS, as this regulation requires certain mandated information on supplier and workplace labels for controlled products (CCOHS, 2016b). Furthermore, many compressed gases lacked a status tag. Specifically, there was no label on the compressed gas cylinders to indicate whether the cylinders were empty, in use or full. This is despite the fact that section 74 of the OHSA requires that empty compressed gas cylinders be labeled as such. As a result of this lack of status tag on the gas cylinders, there may be exposure to unknown risks and unwanted outcomes. These findings show that full compliance to OHSA and WHMIS requirements is lacking in the manner in which chemicals are managed at the labs examined in this study.

Furthermore, this study found that many of the lab chemicals are stored based on alphabetical order, instead of by hazard class. This is a common practice in the laboratories of other universities (Pipitone, 1991; Furr, 2000, pg 
243, and Abbas, Adel, Zakaria, \& Balkyour, 2015). However, it is not in conformance with established lab safety best practices (US National Research Council, 2011b; \& Furr, 2002). Storing lab chemicals alphabetically may cause hazardous chemicals that are incompatible to be stored together. In case of an emergency, accidental mixtures may occur. This may put workers at risk if they are exposed to the hazards in the mixtures. Therefore, it is important to ensure that all lab chemicals are segregated and stored according to their hazard category and compatibility as well as in their proper storage areas. This will help reduce the risk of exposure to hazardous or risky situations when in the lab (US National Research Council, 2011b).

Furthermore, many of the chemicals were stored in fume hoods while not being utilized. Unless in use, fume hoods are not advisable storage areas for chemicals or other lab materials (Foster, 2003; \& Health and Safety Authority, 2012) Storing chemicals in these safety devices may: 1) interfere with or block the airflow in the hoods, preventing the protection of workers from harmful chemicals and their vapours; 2) cause accidental mixtures, since the chemicals stored in fume hoods are generally incompatible with each other; 3) result into hazardous reactions in case a spark or electrical malfunction exists in the fume hood; and 4) contribute to a hood fire (Fred Hutchinson Cancer Research Center, n.d.), which may damage lab equipment and potentially release toxic aerosols. Additionally, storing chemicals or other lab materials in fume hoods may clutter the working space in the hood, making it difficult to safely and efficiently perform lab experiments (Health and Safety Authority, 2012, \& US National Research Council, 2011). In fact, many of the fume hoods, as well as other lab items used for chemical storage (such as shelves, cabinets, etc.) were found in cluttered conditions. In case of a lab emergency, these conditions could also cause incompatible chemicals to accidentally mix and potentially lead to lab damage and/or adverse health and safety impacts on exposed individual.

One way to improve the labeling and storage conditions of chemicals in university laboratories is by establishing an inventory system (Foster, 2005). A positive finding is that University X recently (October 2012) begun managing their chemicals using an online inventory system called HECHMET, by Vertere (E. Ambroise, personal communication, 2016). Specifically, each department was asked to enumerate the chemicals used in their space, in order to have the chemicals logged into the online system. Although this inventory system is ideal for ensuring that there are no surplus chemicals (Weil, 2012), it is unclear if there are frequent physical examinations by lab personnel (supervisor and other lab users) of the chemicals while in their storage areas. Such examinations are necessary to ensure that the chemicals are kept in safe labeling and storage conditions (i.e. no deteriorating or 
missing labels, no leaks, etc.). The findings from this chapter suggest that routine physical chemical inventories may not be performed. If they were, the chemical labeling and storage issues revealed in the 2012-2014 safety reports would not have reappeared in the findings from the current study.

The findings from this study also showed that there might be discrepancies between the hazard(s) and risks associated with the lab chemicals and workers' perception of the risk associated with their lab activities. Specifically, while a small percentage $(9 \%)$ of participants reported that the level of risk associated with their lab work is high, $48 \%$ of the chemicals present in the labs are assigned the 'danger' WHMIS signal word and many of the chemicals were found in poor labeling and storage conditions. While most studies have shown that females have a higher perception of risk, (Flynn, Slovic, \& Mertz, 1994; and Finucane, Slovic, Mertz, Flynn, \& Satterfield, 2000), this was not the case for the participants of the current study. Specifically, the gender difference in lab work risk perception in the current study was not statistically significant. This may be due to the small sample size obtained in this study. However, the chi-square test results indicated that risk perception is significantly affected by the participants' study field or program (i.e. chemistry, biology, and others). In fact, participants from chemistry had a higher perception of the risk associated with their lab work. Nonetheless, it was surprising to find that most participants have a low risk perception about their lab work, while many of the inventoried chemicals were categorized as high-risk substances. This low perception of risk may mislead participants into thinking that they are working in safe lab environments and with non-hazardous substances.

\subsection{Limitations}

Due to the limited amount of time, it was not possible to identify if the lab chemicals are stored longer than permitted. Additionally, due to the clutter issue found in most of the chemical storage areas, it was also impossible to identify all the chemicals stored in the labs examined. Nonetheless, the information obtained from this study still give some understanding about the chemicals handled and stored within University X's chemistry and biology wet labs, their associated hazards, as well as their current storage and labeling conditions.

\subsection{Conclusion}

The purpose of this study was to obtain a better understanding about the nature and safety condition of University X's lab chemicals. Also, the author wanted to see if workers face any health and safety risks when in their labs. It was reassuring to find that workers are not necessarily at any immediate risk from conditions observed in the labs that this study examined. However, we did find some issues of concern such as: 
1) working within the labs potentially involves coming into contact with a lot of unlabeled and improperly stored chemicals, many of which are high-risk substances. If lab personnel come in contact with the unlabeled chemicals or an accidental mixture, they are more likely to become exposed.

2) the University's lab personnel may have an inaccurate perception of the risk(s) associated with their work. However, further examination is needed to solidify this specific conclusion.

Given these findings, it is important to ensure that lab workers from University $\mathrm{X}$ are made aware of the hazards and risks associated with their work. This may prompt lab superiors to put in place appropriate measures for the safe management of their lab substances. For example, 1) establishing a comprehensive laboratory safety program in which proper chemical labelling and storage are addressed, 2) providing safety training that is relevant, as much as possible, to lab personnel's work activities (US National Research Council, 2011b). Another way to make workers aware of the hazards and risks present in their labs is by making available and easily accessible safety reports from audits, inspections, walkthroughs, etc. 


\begin{tabular}{|c|c|c|c|c|c|c|}
\hline Chemical & Flammable? & Corrosive? & Oxidizer? & Toxic/poisonous? & $\begin{array}{c}\text { Compressed } \\
\text { oas? }\end{array}$ & Health hazard? \\
\hline $\begin{array}{l}\text { Potassium and } \\
\text { Sodium Nitrate }\end{array}$ & & & $\checkmark$ & & & $\checkmark$ \\
\hline Diethyl Ether & $\checkmark$ & & & & & $\checkmark$ \\
\hline Helium gas & & & & & $\checkmark$ & $\checkmark$ \\
\hline Chloroform & & & & $\checkmark$ & & \\
\hline Acetylene Gas & $\checkmark$ & & & & $\checkmark$ & $\checkmark$ \\
\hline $\begin{array}{r}\text { Sodium } \\
\text { Borohydride }\end{array}$ & $\checkmark$ & $\checkmark$ & $\checkmark$ & & & $\checkmark$ \\
\hline Methanol & $\bar{v}$ & & & $\checkmark$ & & \\
\hline Hydrogen gas & $\checkmark$ & & $\checkmark$ & & $\checkmark$ & $\checkmark$ \\
\hline Oxygen gas & & & $\checkmark$ & & $\checkmark$ & $\checkmark$ \\
\hline Sulfuric Acid & & $\checkmark$ & & & & $\checkmark$ \\
\hline Acetone & $\checkmark$ & & & & & $\checkmark$ \\
\hline
\end{tabular}




\begin{tabular}{|c|c|c|c|}
\hline \multicolumn{2}{|c|}{ Chemicals\#1 } & \multicolumn{2}{|c|}{ Chemicals\#2 } \\
\hline Name & Hazard class & Name: & Hazard class \\
\hline Dimethylglyoxime & Flammable solid and toxic & Cupric Nitrate & Oxidizing and corrosive \\
\hline Methanol & Flammable and toxic & Chlorine & Corrosive and oxidizing \\
\hline Acetic acid & Flammable and corrosive & Nitric acid & Oxidizing liquid \\
\hline Sodium hydroxide & Corrosive & Isopropyl Alcohol & Flammable \\
\hline Tetrabutylammonium & Oxidizing & Toluene anhydrous & Flammable \\
\hline Sodium Chlorate & Oxidizing solid & Triethylorthoformate & Flammable liquid \\
\hline N-butyllithium solution & Flammable & Hydrogen peroxide & Oxidizing \\
\hline Sulfuric Acid & Inorganic acid (corrosive) & Acetic Acid & Organic acid (Corrosive) \\
\hline Reagent alcohol & Flammable liquid & Hydrochloric acid & Strong acid (Corrosive) \\
\hline Sodium borohydride & Flammable & Sodium perchlorate & Oxidizing \\
\hline Acetonitrile & Flammable liquid & Hydrobromic acid & Strong acid (Corrosive) \\
\hline Chloroform & Toxic & Acetone & Flammable \\
\hline Sodium dodecyl sulfate & Flammable solid & Ammonium persulfate & Strong oxidizer \\
\hline Potassium periodate & Oxidizing material & Sodium borohydride & Flammable, toxic, corrosive \\
\hline Potassium bromate & Oxidizing mater & Sodium sulfite & Flammable, toxic, corrosive \\
\hline Phosphoric acid & Corrosive & Nitric acid & Oxidizing material \\
\hline Hydriodic acid reagent & Corrosive & Ethyl acetate & Flammable \\
\hline
\end{tabular}


Figure 2.1 WHMIS Hazard levels of University X's wet labs chemicals

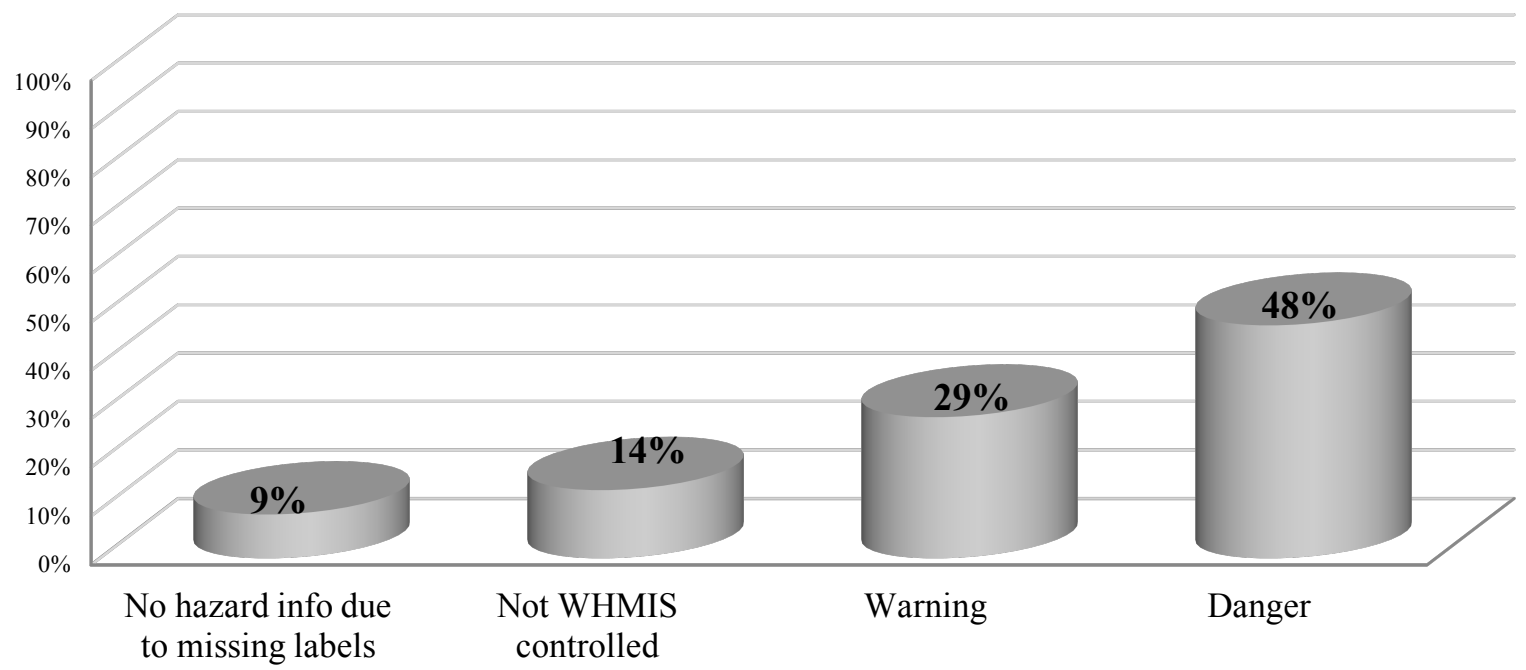

Chemicals hazard levels

Figure 2.2 Chemicals, lab wastes, and lab items stored in fume hoods

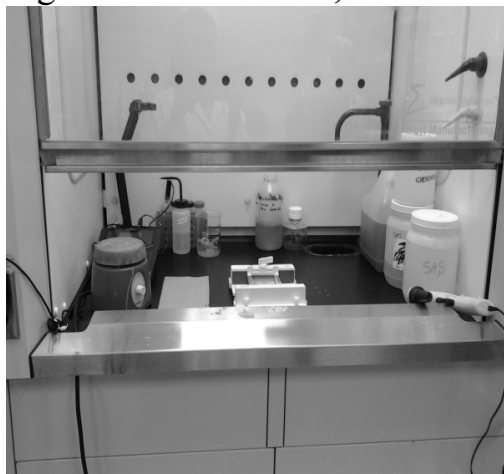

Figure 2.2a.

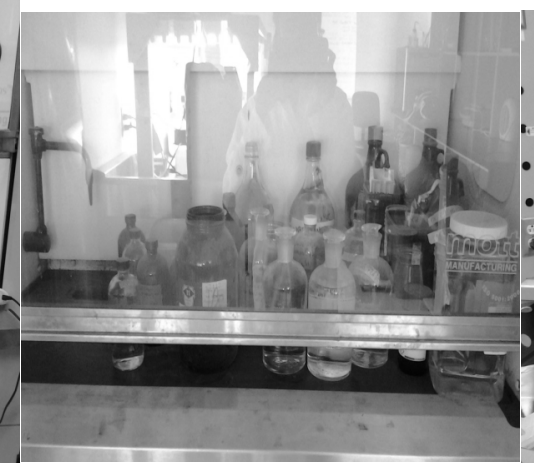

Figure 2.2b.

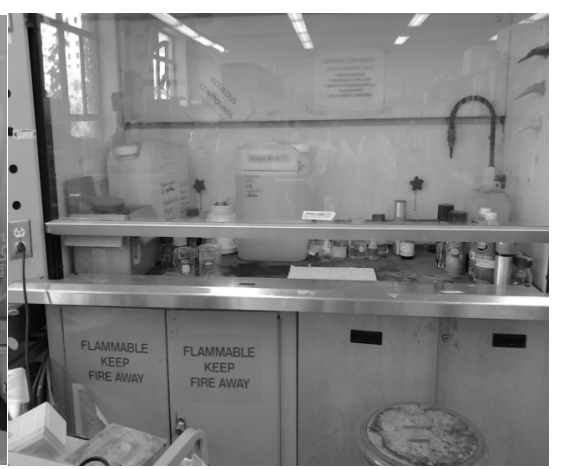

Figure 2.2c

Figure 2.3 Cluttered chemicals on storage shelves

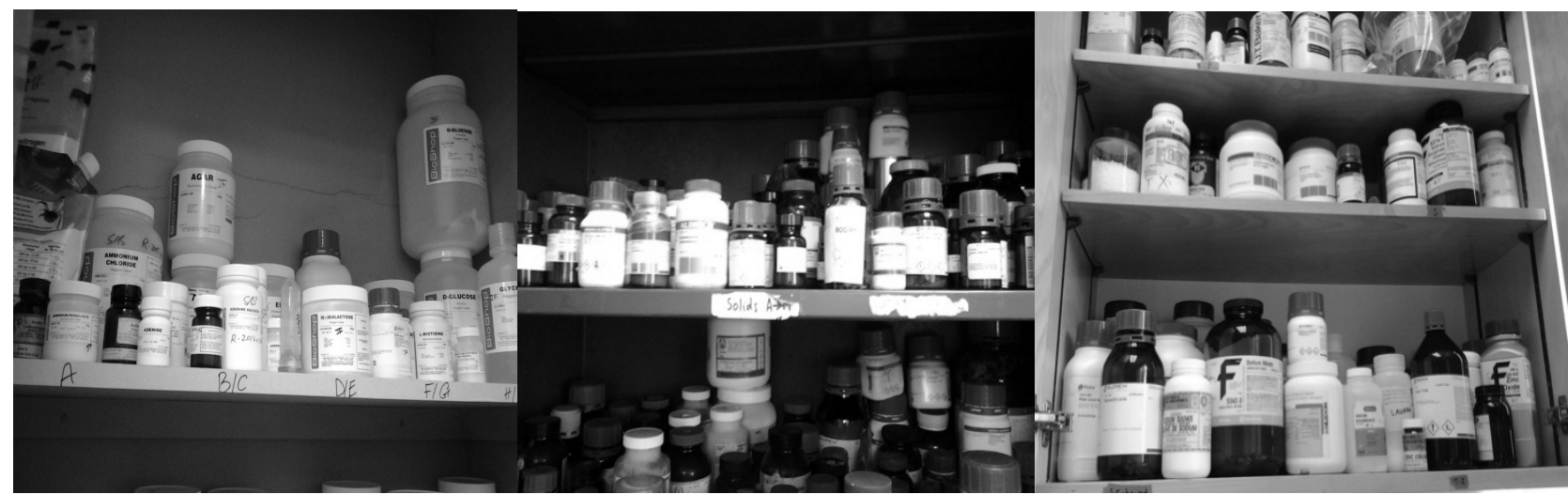


Figure 2.4 storage areas Unclean

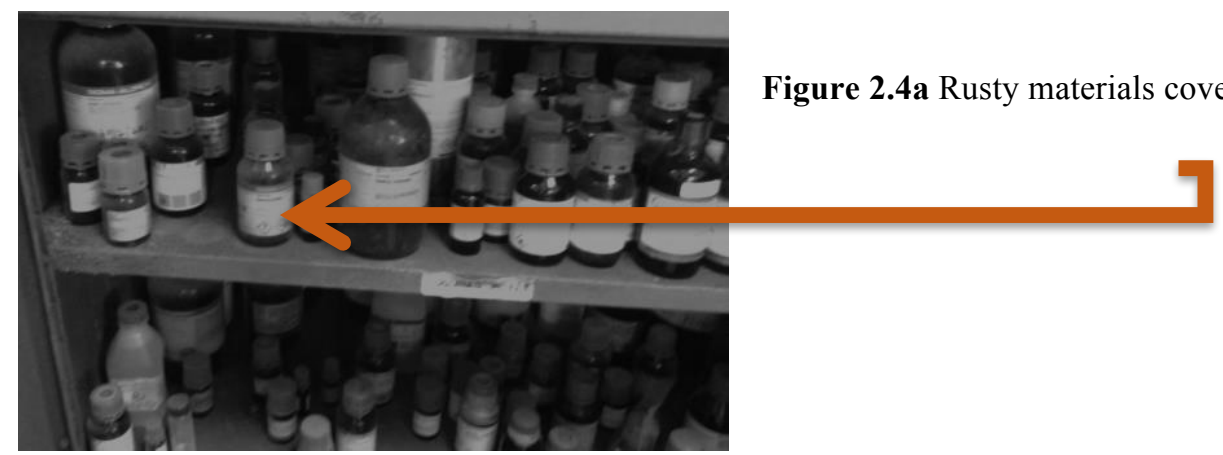

Figure 2.4b Flammable cabinet full of rusty materials

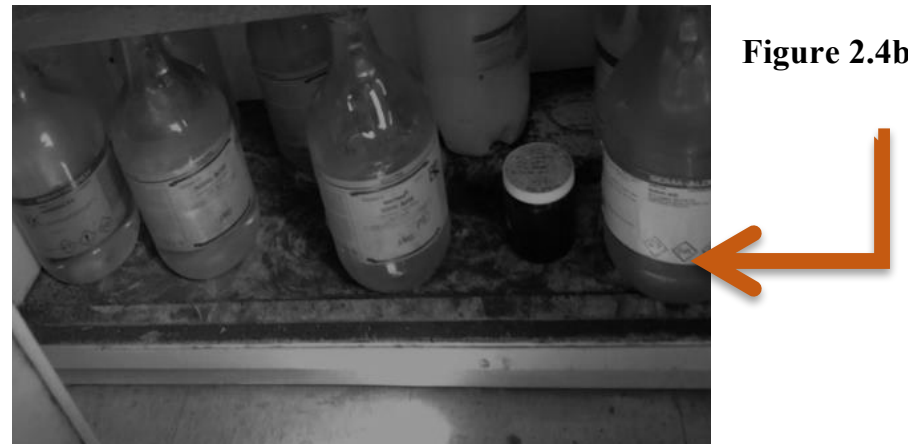

Figure 2.4c Flammable cabinet full of rusty materials and dried leaks, some
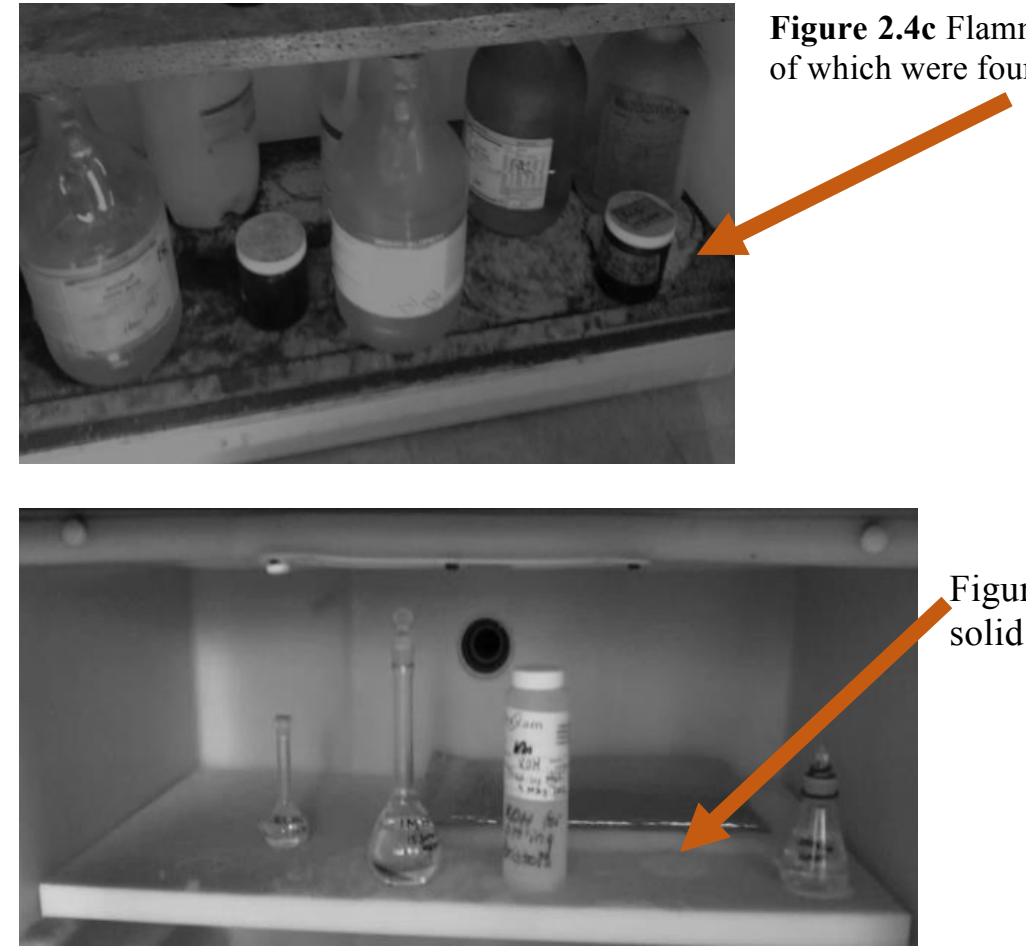

Figure 2.5 Close to empty acids cabinet shelf with spilled solid chemical chemicals on the shelf 


\section{Chapter 3- Identification of safety deficiencies within examined Chemistry and Biology wet labs}

\section{Introduction}

The potential exposure to health and safety hazards, and dangerous conditions make university wet labs unique environments for work and study (Gutiérrez et al., 2013b). However, with the appropriate measures in place, the risk of exposure to hazards can be minimized or even prevented (University of California Riverside, n.d.). University lab personnel are generally students, many of whom are inexperienced and, thus, more prone to injuries (Adane \& Abeje, 2012). Therefore, it is crucial to ensure that the labs are equipped with good working equipment, and that workers are provided with the tools necessary to help them safely carry out their lab activities (Government of Ontario, 2014). Refer to Table 3 for some examples of these materials and equipment.

Unsafe working conditions in a lab can arise under the following circumstances: 1) lab equipment is unavailable; 2) equipment not in good working condition; and 3) improper utilization of equipment. One of the common tools used to identify unsafe lab work conditions is safety inspections (Foster, 2003; Rengarajan, 2012; and Wyllie et al., 2016). In fact, according to the founder of the Laboratory Safety Institute, a provider of safety services

to university and industry labs, "Frequent and regular lab safety inspections are the only hope for spotting problems (i.e. lab hazards or related safety issues)" (Borchardt, 2006). Additionally, according to Wyllie et al (2016), the information obtained from inspections is one of the most credible safety indicators available today.

\subsection{Safety Inspections and University wet lab conditions}

Safety inspections are based on regulatory requirements (Government of Ontario, 2014a; \& Rengarajan, 2012). They are usually designed in the form of checklists in which the following topics are covered: housekeeping, personal protective equipment (PPE), chemical storage and waste management, signs and labels, safety documents, safety devices, emergency safety equipment, etc. (Foster, 2003 and 2007). The results from safety inspection are what investigators use to 1) inform lab supervisors or owners of any deficiencies that may be present in their lab, and 2) provide them with corrective actions, which when acted upon can help enhance the lab's safety conditions (Government of Ontario, 2014a). Periodic inspections help maintain the laboratory environment and equipment in safe working conditions for all personnel (US National Research Council, 2011b). Despite the important role that inspections play in lab safety, the results of an international study (BioRaft, Nature Publishing Group, \& University 
of California Center for Laboratory Safety, 2012), revealed that $21 \%$ of the participants, the majority of whom were from academic institutions, did not even know if safety inspections were performed in their labs.

Recently, various U.S. universities have experienced numerous accidents in their wet labs (Wyllie et al., 2016). Some of these accidents could have been prevented if corrective actions suggested from past inspection findings were addressed. For example, after the 2008 fatal accident at the University of California Lost Angeles (UCLA) (Van Noorden, 2011; and Schröder et al., 2014), reports showed that two months prior to the incident, the lab was inspected several times and numerous safety deficiencies were identified (Kemsley, 2009); \& Christensen, 2009). Some of the deficiencies included lack of protective equipment, improper storage of chemicals, etc. Although the lab supervisor was informed about the deficiencies, no corrective actions were taken to address the safety issues. Therefore, some suggested that if the supervisor had dealt with the identified issues, perhaps the accident could have been prevented (Eldridge, 2012). Due to many other similar accidents, today's academic wet labs are considered unsafe environments for work and study. In fact, according to the founder of Laboratory Safety Institute (2014), the conditions in today's wet labs are "shameful, horrifying, and notoriously bad" (1978b).

As demonstrated in Chapter 1, there are numerous hazardous chemicals present within the wet laboratories of the medium-sized Canadian academic institution (University $\mathrm{X}$ ) examined in this research. As previously mentioned, many of these chemicals are in unsafe labeling and storage conditions. However, it is unknown if there are other safety issues present within the chemistry and biology labs that were examined. Also, since the University has established a Workplace Inspection Program, it is uncertain how effective this program is in ensuring that lab safety inspections are routinely conducted. Therefore, the goal for this chapter was to identify safety deficiencies that may be present within the labs.

\subsection{Chapter 3 objectives}

This chapter has the following six specific objectives:

1) identify the frequency of safety inspections within the labs,

2) identify the state of housekeeping of the labs,

3) identify whether safety documents (e.g. safety manuals and Safety Data Sheets (SDSs)) are available,

4) identify whether safety equipment and devices (e.g. PPE, chemical fume hoods and biosafety cabinets) are available and in good working condition,

5) identify the manner in which hazardous waste materials are managed within the labs 
6) identify if concerns revealed in past safety reports (i.e.2013-2014 inspections) have been addressed.

\subsection{Materials and Methods}

Before starting this study, ethics approval was obtained from University X's Research Ethics Board. Lab and subject recruitment was performed via emails, in person, and by posting of flyers within several buildings of the University. The lab participation criterion was: any chemistry and biology wet lab that is currently and actively utilized for research and teaching. The subject inclusion criterion was: any member of University X (students, faculty, and staff) currently engaged in chemistry and/or biology wet lab activities within the University.

To obtain an understanding of how often safety inspections are performed for the University's wet labs, participants holding supervisory roles were asked in an online survey to indicate the frequency of safety inspections in their labs. The remainder of this study was carried out by inspecting all participating chemistry and biology wet laboratories. The inspections were conducted using checklists developed based on existing inspection forms obtained from various universities (Foster, 2003); Washington DC, n.d. ; University of Florida, 2016; WorkSafe BC, 2008; University of Memphis, n.d.; University of Washington Environmental Health \& Safety, 2015; and University of Washington, n.d.). Two sets of checklists were developed: one focused on housekeeping, PPE, Safety and Emergency Equipment, Signage, Emergency safety information and lab security (Appendix C); and the other focused on hazardous waste management (Appendix D). Both checklists were divided in two parts, A and B.

Most of the questions in part A were written in an open-ended format and focused on the availability of lab

safety manuals, SDSs, and disinfectants. These data were obtained from supervisors, technicians, or designated knowledgeable lab personnel. The items in part B were written in a multiple choice format. Part B data were collected by 1) observing each lab to obtain answers for the inspection items, and 2) recording the observations on the checklist form.

Lastly, to identify if there are any reoccurring safety issue(s), some the findings from this study's inspections were compared with the summary results from the 2013-2014 inspections. All study data was descriptively analyzed.

\subsection{Results}

Out of 34 chemistry and biology wet laboratories reached, only $22(65 \%)$ participated in this study. For the survey, out of approximately 159 individuals reached, 61 consented to participate. However, among those who consented to participate, five skipped the entire survey, resulting in a response rate of $35 \%(56 / 159)$. It should be 
noted that $84 \%(47 / 56)$ of the participants came from chemistry and biology study fields, while $16 \%(9 / 56)$ came from other departments i.e. engineering, etc.

\subsubsection{Safety data records and inspections frequency of University $X$ 's wet labs}

Table 3.2 shows that $43 \%(6 / 14, n=5$ Biology participants, $n=1$ Chemistry participants $)$ of the survey participants who hold supervisory roles are not aware of how often inspections are carried out in their labs. Also, $29 \%(4 / 14, n=3$ Biology, $n=1$ Chemistry $)$ of lab superiors disagreed that they have access to any tracked or recorded data regarding their lab's state of safety and level of compliance.

\subsubsection{Housekeeping within the laboratories}

As shown in Table 3.3, 32\% of the labs had their entrance/exit doors opened rather than closed. Regarding cleanliness and order, this study found the aisles in $45 \%$ of the labs obstructed with power cords, cardboard boxes, chemical drums, waste containers, etc. These objects made it difficult to walk freely in the aisles. Additionally, $50 \%$ of the labs had the following non-lab related items on their benches: worker's bags, clothes, etc. The floor in $41 \%$ of the labs were found in unclean conditions i.e. floors had visible dried stains and debris. Overall, $82 \%$ of the labs were found in an unorderly condition.

Although $77 \%$ of the labs were equipped with cleaning and decontamination supplies, $68 \%$ of the chemical fume hoods had visible dried stains and debris, spilled chemical powders, and some rusty spots. Also, $32 \%$ of the labs had a lot of stains and some debris on their bench-tops.

\subsubsection{Lab safety documents and materials}

Table 3.4 shows that $68 \%$ of the labs do not have a laboratory safety manual. Additionally, although a majority of research labs were equipped with Safety Data Sheets(SDSs), these essential documents were not found in $23 \%$ of the teaching labs. In fact, the research team was informed that the SDSs are "somewhere online". Furthermore, many of the SDSs in research labs dated were more than three years old.

\subsubsection{Lab Safety Devices, Emergency and Proper Protective Equipment(PPE)}

As shown in Table 3.5, lab coats and gloves were lacking in 55\% of the labs. This issue was mostly observed in the teaching labs. Additionally, only $18 \%$ of the labs were equipped with safety glasses. This was mostly found in chemistry research labs. It should be noted that at the time of the inspection, the research team was verbally informed that students from teaching labs store their coats, gloves, and safety glasses outside the labs i.e. in lockers assigned to them individually (S. McFadden, personal communication, 2016). Furthermore, although most 
of the research labs were equipped with protective coats, $70 \%$ of their coats hanging in areas unseparated from street clothes.

Table 3.5 also shows that $45 \%$ and $36 \%$ of the labs are not equipped with safety showers and eyewash stations, respectively. Among the labs that had these two safety devices, $50 \%$ were obstructed by boxes, bench stools, and waste containers. Though the labs equipped with the safety showers and eyewash stations had an inspection tag, there was no indication on the tags that the equipment had been tested or inspected within the last year. Similarly, nothing was written on the inspection stickers found on the fume hoods in $45 \%$ of the labs.

Regarding emergency equipment, fire extinguishers in $41 \%$ of the labs were found obstructed by refrigerators, broken glass waste containers, and other large objects. Despite the fact that all inspected labs had numerous hazardous chemicals (as previously mentioned in Chapter 1), a stocked spill cleanup kit was not found in $95 \%$ of the labs.

\subsubsection{Hazardous wastes management within University $X$ wet labs}

According to Table 3.6, 36\% of the labs had hazardous and non-hazardous waste materials stored beside each other, a non-recommended practice. Also, $45 \%$ of the labs did not have their flammable and non-flammable waste materials stored away from each other. Additionally, in $23 \%$ of the labs, acid and base residues were found stored beside each other. Furthermore, $27 \%$ of the labs equipped with chemical fume hoods had many of their hazardous liquid wastes stored in the hoods. Similarly, the biosafety cabinets in $14 \%$ of the biology labs were used as storage areas for the lab's hazardous waste materials. Regarding the waste containers themselves, corrosion spots and dried leaks were found on some of the vessels in $41 \%$ of the labs. In $18 \%$ of the labs, some waste containers were found stored in egress routes i.e. entrance/exits and passageways.

\subsubsection{Recurring safety issues within the labs}

Many of the deficiencies identified in University $\mathrm{X}$ via past safety reports (i.e. walkthrough, audit and inspections) reappeared in this current study. For instance, the 2013-2014 inspections identified that the eyewash stations in many of the research (78\%) and teaching $(65 \%)$ labs are not regularly tested. Similarly, this current study revealed that none of the eyewash stations in the examined research and teaching labs were routinely inspected. Additionally, both inspections revealed that spill kits are not provided in most of the labs. Findings from the 2012 laboratory walkthrough and this study revealed many SDSs dating more than three years. 


\subsection{Discussion}

The goal of this study was to examine the safety conditions that currently exists within University X's chemistry and biology wet laboratories. It was reassuring to find that, similar to other academic institutions (Wyllie et al., 2016), this University has established a Workplace Inspection Program, which requires all campus premises to be annually inspected. However, the various safety deficiencies (Figure 1) identified in this study makes one wonder 1) about the effectiveness of this program, and 2) whether it is being enforced to ensure that lab safety inspections are routinely carried out and that identified issues are addressed.

Furthermore, the fact that many survey participants who hold supervisory roles could not tell how often their labs are inspected, suggests that frequent inspections may be lacking or nonexistent in their labs. This shows a potential lack of compliance with section 6 of the Occupational Health and Safety Act, which requires that annual safety inspections be carried out in laboratories and other workplaces (Government of Ontario, 2014a). Thus, lab superiors may lack full awareness about the safety deficiencies that might be present in their work environment. Through these discussions lab personnel learn about how they can identify hazards and safe work practices in their workplace(Wyllie et al., 2016). Furthermore, the fact that many lab superiors lacked access to any data or records about the safety compliance of their labs, indicates that they may not be aware about the state of safety within their labs. This makes it difficult and almost impossible for lab superiors to inform their workers about the safety conditions of the labs and put in place appropriate control measures, as is required by sections $25-27$ of the Act (Government of Ontario, 2014a).

Given that hazardous substances are handled in the labs, it was surprising to find that the majority $(68 \%)$ of the labs lacked a laboratory safety manual. This is contrary to the practice at other universities, many of which were found equipped with such safety documents (University of Guelph, 2013; Iowa State University, 2011; McMaster University, and 2008, Simpson, 2005). Although having a lab safety manual is a general best practice, it is indirectly implied by the OHSA, which asks employers and/or supervisors to ensure written instructions are made available to lab personnel for the safe conduct of their work (Government of Ontario, 2014a). Additionally, lab safety manuals provide the necessary information and supporting documentation to help educate workers about the "dos and don'ts" of working in a lab setting and ensure that activities are safely carried out (Rahman, 2010; \& Hancock, 2014). The fact that fume hoods were used as hazardous waste storage areas in many of the inspected labs shows that workers are not entirely aware of the safe way to store their lab wastes. 
The concern with storing hazardous waste in fume hoods is that it may interfere with air-flow, generate toxic aerosols, reduce the working space in the hood, result in hood fires, etc. (Fred Hutchinson Cancer Research Center, n.d.). In fact one of the main factors that contributed to the 2003 hood fire at the National Renewable Energy Laboratory was hazardous waste materials (such as cleaning wipes damp with flammable chemicals and waste pipettes containing an air-sensitive chemical) left unattended in the front area of a chemical fume hood (Cotter $\&$ Whitherspoon, 2013; and University of California Santa Barbara, 2014).

Similar to the findings from other universities (Abbas, Adel, Zakaria, \& Balkyour, 2015), this study found that SDSs are physically lacking in some of the examined teaching labs. Having SDSs readily available is not just a regulatory requirement; but it ultimately helps inform workers about the actual or potential dangers and risks associated with their lab substance(s), and educates them on the appropriate measures to employ for the safe handling of those substances (Canadian Centre for Occupational Health and Safety, 2016b). Therefore, when SDSs are absent or inaccessible, lab personnel's OHSA right to know about the hazards and risks associated with their work is violated. As a result, workers may lack full awareness about the dangers and level of risk involved with their lab chemicals.

Furthermore, WHMIS also requires employers to ensure that their SDSs are up-to date to reflect any newly discovered hazard or condition about their chemicals. The fact that many (59\%) of the SDSs from the research labs were more than ten years old raises questions about how accurate the current understanding of the hazards and risks associated with the lab chemicals is. Also, the signs of corrosion found on many of the chemical containers (see figure 5 from Chapter 1) might indicate that the chemicals associated with the SDSs have expired. If this the case, they should be removed and safely disposed of; as there may be adverse outcomes when lab substances are stored for extended periods of time. For example, reports from the Ontario Ministry of Labour revealed that in 2013, workers from an environmental facility suffered various injuries from an explosion of lab samples that had been stored for long periods of time (Government of Ontario, 2016b).

When it comes to safety emergency equipment, sections 124, 125 and 137 of the Industrial Establishments Regulations (R.R.O. 1990, Reg.851) under the OHSA requires that every premise where hazardous substances are handled be provided with eyewashes and safety showers (Government of Ontario, 2016). Given that many of the inventoried chemicals were high-risk substances, it was surprising to find that many of the labs lacked safety showers and eyewash stations (45\% and 36\%, respectively). This shows non-compliance with the requirements of 
the Act. Further findings from this study revealed that the labs that had chemical fume hoods, biosafety cabinets, and eyewash stations had blank inspection tags on many of these safety/emergency devices. This suggests that these safety devices lack regular inspections, which are required by various standards (e.g. the American National Standards Institute (ANSI/AIHA Standard Z95), National Fire Protection Association (NFPA 45), Canadian Standards Association (CSA Z316.3-95), and National Sanitation Foundation (NSF/ANSI 49)) (CCOHS, 2017; Public Health Agency of Canada, 2005; and Ciloski,2005).

Given the potential exposure to the hazards and risks associated with majority of the lab chemicals (see Chapter 1), it was also surprising to find that many of the labs lacked protective coats, gloves, and eye-wear. This shows a potential breach of sections 81 and 84 of the Industrial Establishments Regulations (R.R.O. 1990, Reg.851), which require that workers exposed to the hazard of eye and skin injuries be protected with appropriate barriers (Government of Ontario, 2016). Furthermore, having students store their PPE outside the lab environment (S. McFadden, personal communication, 2016) may facilitate these non-lab items be stored with potentially infected or contaminated PPE. Additionally, the fact that many of the research labs equipped with safety coats had these hanging in the same area as street clothes shows lack of conformance with lab best practices ( CDC, NIOSH, \& U.S. Consumer Safety Product Commission, 2006; and Concordia University, 2017.). Therefore, it is necessary to ensure that lab workers are well trained in the safe handling of their work substances.

Safe lab practices can help minimize or even prevent spills and other lab emergencies. Nonetheless, should a hazardous spill occur in any of the labs, sections 4.1.6 and 4.12.5.1 of the Ontario Fire Code (O. Reg. 213/07) requires that that 1) a written spill control procedure, and 2) appropriate equipment/materials necessary to contain and safely clean up the spill be provided (Government of Ontario, 2015). However, the lack of spill cleanup kits in $95 \%$ of the labs is a sign of non-compliance with this legislated Code (Government of Ontario, 2015). In addition to showing a lack of compliance with regulatory requirement, this finding suggests that, in the event of a lab spill, workers from majority of the labs that were inspected may not be well equipped to adequately address such an emergency.

Since many of the participants confirmed that they have cleaning and decontamination supplies for use before and after lab operations, it was unexpected to find many of their work surfaces (i.e. bench-tops, chemical fume hoods, and some biosafety cabinets) in unclean conditions i.e. stained, had visible dust and debris, etc. This implies that the available cleaning and decontamination supplies may not be routinely utilized by the lab personnel. The 
importance of using cleaning and decontamination supplies on surfaces or other lab work areas is that it helps minimize exposure to hazardous substances (Burnett, Lunn, \& Coico, 2009). Since many of the labs lacked either gloves or safety coats, not using the cleaning or decontamination supplies may lead to the spread of contaminants among workers and, possibly, to the general public. Therefore, it is essential that workers make full use of the safety materials available in their labs.

Regarding order in the labs, the cluttered or crowded conditions found in many of the labs may facilitate exposure to trips and fall hazards. This may injure lab workers and students (Sattin, Rodriguez, DeVito, and Wingo, 1998), especially when there's an emergency that requires quick evacuation (i.e. fires or a major hazardous spill). Another housekeeping issue identified by this study is the fact that many of the inspected labs had their doors open. It is generally a best practice to keep lab doors closed at all times. Keeping them closed helps contain toxic vapours, and airborne contaminants from entering non-lab areas to adversely impact the building air quality as well as the health and safety of the building occupants (Walters, 2005; US National Research Council, 2011; Government of Ontario, 2014a; and Brigham Young University, 2016). Since hazardous chemicals are handled within the University's chemistry and biology wet laboratories, it is very important to ensure that the lab doors are kept closed at all times.

After comparing the findings from this current study to that of the previously mentioned 2012 to 2014 safety reports, it was surprising to still find numerous recurring safety issues. This gives the impression that either: 1) corrective actions were not recommended and reported to lab superiors, as is required by the OHSA; or 2) if they were, not all of them were addressed or rectified. Nonetheless, the reappearance of certain safety issues not only shows a lack of compliance with OHSA, but also suggests that the Internal Responsibility System may not be well integrated and functioning at its full potential within the University. Therefore, it is crucial that every lab stakeholder within their respective capacity take the necessary precautions to ensure that safety issues identified from inspections or other safety studies are addressed before any undesirable emergency occurs. However, it is still the legal obligation of the University and those who fall under the category of "supervisors" to ensure that inspections are routinely carried out for their lab space and that control measures are put in place to address any identified safety issue(s). 


\subsection{Study limitations}

Although the results of the inspection represent a point in time only, they are meaningful. They indicate that there is evidence of non-compliance at the University. Furthermore, many of the same issues identified in the current study were raised in the 2012 laboratory walkthrough, safety audit, and 2013-2014 inspections.

\subsection{Chapter conclusion}

This chapter examined the safety conditions that exist within some of the wet labs of University X. Many safety deficiencies, some of which were reoccurrences from previous safety visits (i.e. 2013-2014 inspections), were identified. These deficiencies were a result of non-compliance with OHSA requirements and non-conformance to general lab best practices. Some of the safety violations include: 1) safety inspections may be infrequently or not at all carried out in some of the labs; 2) many of the labs lacked a safety manual, spill clean-up kits, safety showers, eyewash stations, and regularly inspected fume hoods; 3) some of the labs had many of their coats stored in the same area as street clothes or other non-lab items; and 4) many of the labs had their doors open. Although these deficiencies do not necessarily put the labs examined at imminent danger, they may contribute to injuries or accidents if they are not rectified. Furthermore, while it is crucial for all lab stakeholders to actively participate in keeping their work environment safe as per the Internal Responsibility System, the ultimate responsibility for ensuring that inspections are conducted and that lab safety concerns are addressed lies with employer (the academic institution) and supervisors (departmental chairs, deans, and Principal Investigators etc.) as per the OHSA. Therefore, it is strongly recommended that the University ensure that its Workplace Inspection Program is implemented so that lab inspections can be carried out routinely and that identified safety deficiencies can be rectified, as required by the OHSA. An excerpt from the program can be found in appendix E. 
Table 3.1: Examples of essential lab safety items (safety manuals, equipment, and safety devices)

\begin{tabular}{|l|l|}
\hline \multicolumn{1}{|c|}{ Items } & \multicolumn{1}{|c|}{ Purpose/Importance of item } \\
\hline Up-to-date Safety Data Sheets (SDSs) & $\begin{array}{l}\text { Inform lab worker of the hazards and risks that may be associated with their lab } \\
\text { substances as well as safe handling measures }\end{array}$ \\
\hline Lab safety manuals & $\begin{array}{l}\text { Further enhance workers' knowledge about specific lab hazards } \\
\text { Provide safe operating procedures for to help protect those working with hazardous } \\
\text { substances }\end{array}$ \\
\hline $\begin{array}{l}\text { Proper Protective Equipment i.e. gloves, lab coat, eye } \\
\text { protection, etc. }\end{array}$ & $\begin{array}{l}\text { Necessary when working with or in an environment where hazardous substances are } \\
\text { handled }\end{array}$ \\
\hline $\begin{array}{l}\text { Safety devices i.e. Chemical fume hoods and biosafety } \\
\text { cabinets }\end{array}$ & Serve as containment devices for the safe handling of very hazardous substances \\
\hline Safety showers, eyewash stations, spill clean-up kits & Enable lab workers to promptly respond to lab incidents i.e. fires, spills, etc. \\
\hline
\end{tabular}

\section{Table 3.2: Information about participating wet labs from University X}

\section{Labs information}

Lab type
Research: chemistry labs

Research: biology labs

Teaching: chemistry labs

Teaching: biology labs

How frequently are safety inspections carried out for your lab?

At least once a month

At least once a quarter

At least once per year

I don't know

\section{Participating labs}

$9 \%$

$41 \%$

$27 \%$

$23 \%$

$7 \%$

$14 \%$

$36 \%$

$43 \%$

I have access to the data and records which are tracked regarding your lab's safety and compliance 


\section{Table 3.3: Housekeeping within University x's chemistry and biology wet labs}

\begin{tabular}{|c|c|}
\hline Questions & Response \\
\hline \multicolumn{2}{|l|}{ Are lab doors kept closed? } \\
\hline $\begin{array}{l}\text { Yes } \\
\text { No }\end{array}$ & $\begin{array}{l}68 \% \\
32 \%\end{array}$ \\
\hline \multicolumn{2}{|l|}{ Are the lab passageways free of obstruction? } \\
\hline $\begin{array}{c}\text { Yes } \\
\text { No } \\
\end{array}$ & $\begin{array}{l}55 \% \\
45 \%\end{array}$ \\
\hline \multicolumn{2}{|c|}{ Are cleaning and decontamination supplies available? } \\
\hline $\begin{array}{c}\text { Yes } \\
\text { No } \\
\end{array}$ & $\begin{array}{l}77 \% \\
23 \% \\
\end{array}$ \\
\hline \multicolumn{2}{|c|}{ Are the lab floors clean i.e. No spills or debris on them? } \\
\hline $\begin{array}{c}\text { Yes } \\
\text { No } \\
\end{array}$ & $\begin{array}{l}73 \% \\
27 \% \\
\end{array}$ \\
\hline \multicolumn{2}{|l|}{ Are lab benches clean i.e. No spills on them? } \\
\hline $\begin{aligned} \text { Yes } \\
\text { No } \\
\end{aligned}$ & $\begin{array}{l}68 \% \\
32 \%\end{array}$ \\
\hline \multicolumn{2}{|c|}{$\begin{array}{l}\text { Are lab benches clear of unnecessary items such as worker's bags or home } \\
\text { clothes? }\end{array}$} \\
\hline $\begin{array}{l}\text { Yes } \\
\text { No }\end{array}$ & $\begin{array}{l}50 \% \\
50 \%\end{array}$ \\
\hline \multicolumn{2}{|c|}{ Overall, is the general appearance of the labs orderly? } \\
\hline $\begin{array}{l}\text { Yes } \\
\text { No }\end{array}$ & $\begin{array}{l}18 \% \\
82 \%\end{array}$ \\
\hline
\end{tabular}

\section{Table 3.4: Lab safety documents}

\begin{tabular}{|c|c|}
\hline \multicolumn{1}{|c|}{ Safety topic } & Responses \\
\hline Is there a lab safety manual available that the lab follows? & $32 \%$ \\
Yes & $68 \%$ \\
No & \\
\hline Are safety data sheets present in the lab? & $77 \%$ \\
Yes & $23 \%$ \\
No & \\
\hline Are safety data sheets up-to-date (e.g. Less than 3 years old)? & $5 \%$ \\
Yes & $59 \%$ \\
No & $36 \%$ \\
\hline
\end{tabular}


Table 3.5: Availability and safety condition of Personal Protective Equipment and Safety Devices within University x's wet labs

\begin{tabular}{|c|c|}
\hline PPE and Safety Devices & Responses \\
\hline \multicolumn{2}{|l|}{ Are lab coats available in the lab? } \\
\hline $\begin{array}{ll}\text { Yes } \\
\text { No } \\
\end{array}$ & $\begin{array}{l}45 \% \\
55 \%\end{array}$ \\
\hline \multicolumn{2}{|l|}{ Are the lab coats hanged in a separate area of the lab? } \\
\hline $\begin{array}{l}\text { Yes } \\
\text { No }\end{array}$ & $\begin{array}{l}30 \% \\
70 \%\end{array}$ \\
\hline \multicolumn{2}{|l|}{ Are gloves available in the labs? } \\
\hline $\begin{array}{ll}\text { Yes } \\
\text { No }\end{array}$ & $\begin{array}{l}45 \% \\
55 \%\end{array}$ \\
\hline \multicolumn{2}{|l|}{ Are eye protections available in the labs? } \\
\hline $\begin{array}{l}\text { Yes } \\
\text { No }\end{array}$ & $\begin{array}{l}18 \% \\
82 \%\end{array}$ \\
\hline \multicolumn{2}{|l|}{ Are safety showers available? } \\
\hline $\begin{array}{ll}\text { Yes } \\
\text { No } \\
\end{array}$ & $\begin{array}{l}55 \% \\
45 \%\end{array}$ \\
\hline \multicolumn{2}{|l|}{ Are there eyewash stations in the lab? } \\
\hline $\begin{array}{cc}\text { Yes } \\
\text { No } \\
\end{array}$ & $\begin{array}{l}64 \% \\
36 \%\end{array}$ \\
\hline \multicolumn{2}{|l|}{ Are the eyewash and safety showers obstructed? } \\
\hline $\begin{array}{cc}\text { Yes } \\
\text { No } \\
\end{array}$ & $\begin{array}{l}50 \% \\
50 \%\end{array}$ \\
\hline \multicolumn{2}{|l|}{ Are the lab fume hoods regularly inspected? } \\
\hline $\begin{aligned} \text { Yes } \\
\text { No }\end{aligned}$ & $\begin{array}{l}55 \% \\
45 \%\end{array}$ \\
\hline \multicolumn{2}{|l|}{ Do the labs have spill clean-up kits? } \\
\hline $\begin{aligned} \text { Yes } \\
\text { No }\end{aligned}$ & $\begin{array}{l}5 \% \\
95 \%\end{array}$ \\
\hline \multicolumn{2}{|l|}{ Are the lab fire extinguisher obstructed? } \\
\hline $\begin{array}{l}\text { Yes } \\
\text { No }\end{array}$ & $\begin{array}{l}41 \% \\
59 \%\end{array}$ \\
\hline
\end{tabular}




\section{Table 3.6: Hazardous Waste Management within University x's wet labs}

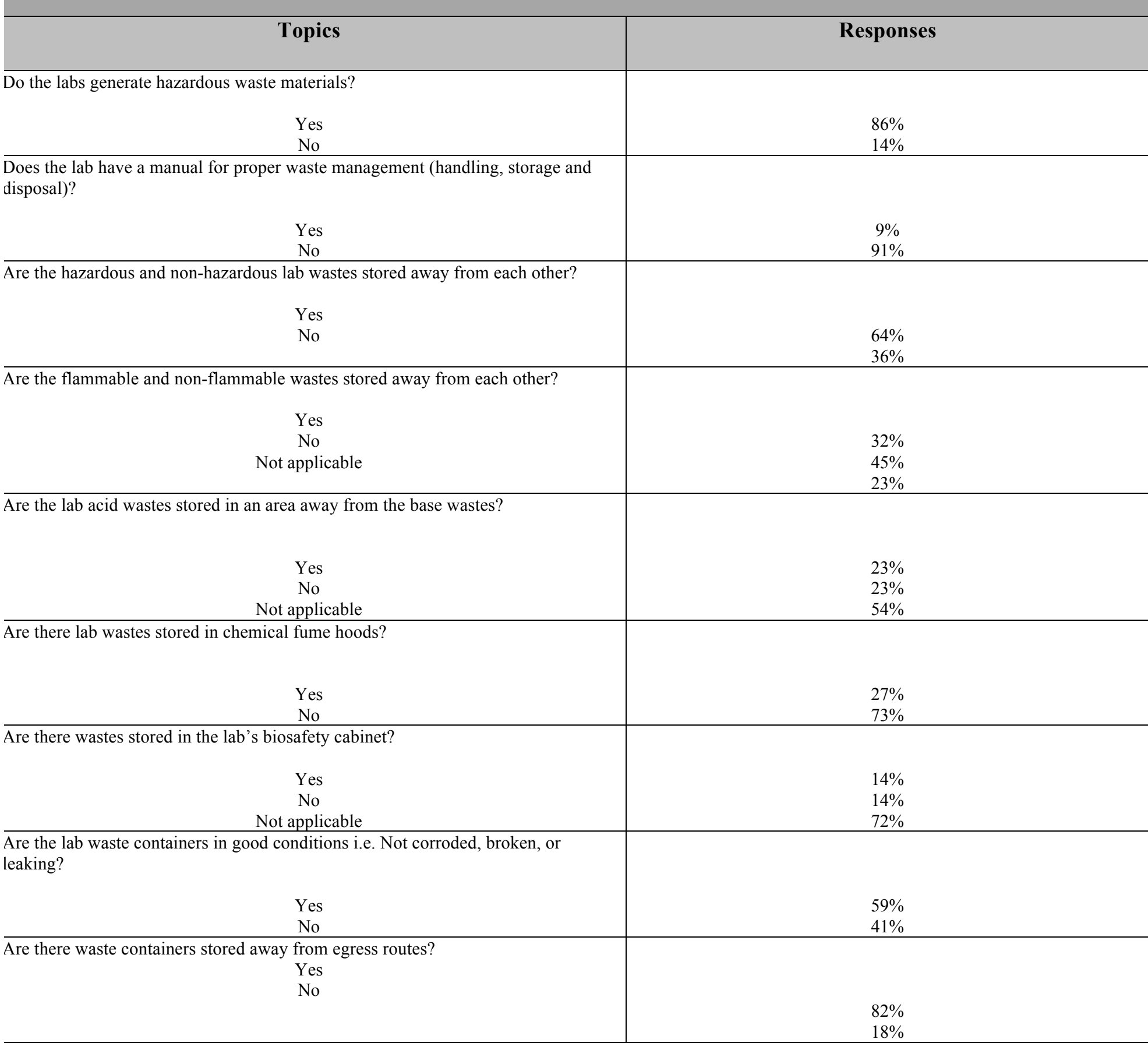


Figure 3.1. Laboratory safety deficiencies identified after 2016 safety inspections

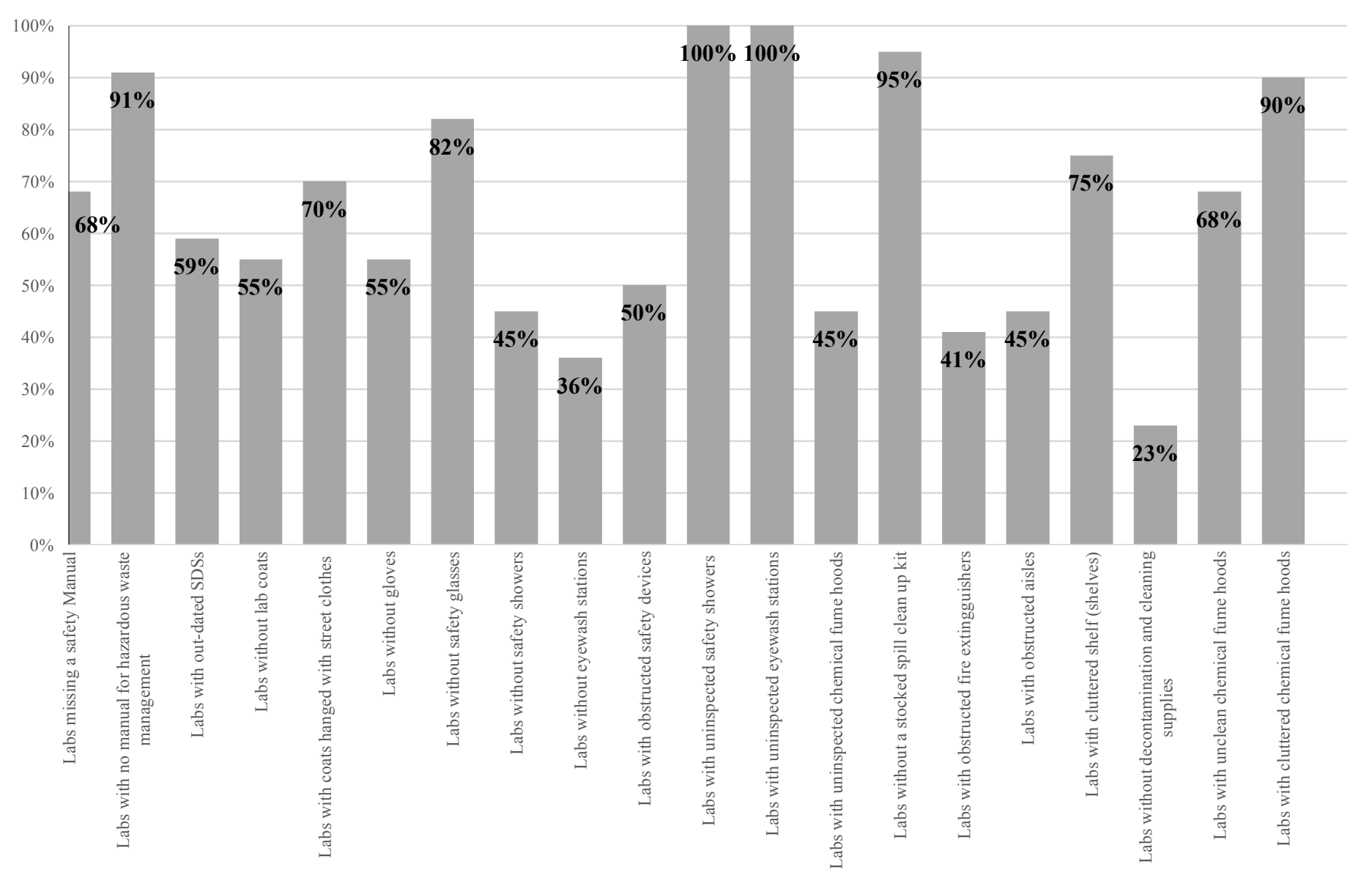




\section{Chapter 4- Safety attitudes and practices of University X Lab Personnel}

\section{Introduction}

Scientific education and research, many of which are provided in academic institutions, help enhance the quality of life, as well as our means of communication, transportation, food, etc. (Asimov, Burke, \& Bergman, 2013). However, working and studying in university wet laboratories involves the handling of hazardous substances. Due to the dangerous properties associated with these substances (Adane \& Abeje, 2012; (US National Research Council, 2011b; and Langerman, 2009), it is crucial that those handling them do so safely. When these substances are not safely managed, accidents are likely to occur and, as a result, lab workers run the risk of having their health and well-being adversely impacted. This may lead to the reduction of lab work productivity, which may potentially hinder the furthering of scientific research and ultimately bring societal advancements to a halt. One of the factors that can cause unsafe management of lab substances is when safety is not practiced as a shared responsibility in which all workers in an organization play a significant role to ensure that their respective work environments are safe for learning and conducting research (Ministry of Labour, 2015). The presence or absence of this shared responsibility is generally identified by examining the lab's state of safety culture.

\subsection{Safety culture within university wet labs}

The term 'safety culture' signifies a "set of values, norms, roles, technical practices, etc., that are concerned with minimizing the exposure of employees, managers, members of the public to dangerous or injurious conditions" (Cooper, 2000, and Hill et al., 2012). A workplace with a strong and positive safety culture thrives on 1) a substantial commitment from all workers to operate safely and 2) a high priority placed on safety at all times (US National Research Council, 2014). The benefit of a strong and positive safety culture is that it helps identify and appropriately mitigate all foreseeable and unforeseeable lab hazards (US National Research Council, 2014). The rewarding outcomes of a strong and positive safety culture are not only seen at the university level but ultimately on society as a whole, by having healthier scientists perform ground-breaking research for the betterment of society.

Over the years, numerous wet lab accidents have been reported in various universities globally. As a result, an international safety culture survey (Van Noorden, 2013\& Schroder et.al, 2016) was conducted in a joint collaboration between Nature Publishing Group (NPG, the publisher for the Nature Journal), University of California (UC) Center for Laboratory Safety, and BioRaft (a firm that provides software for safety compliance) (Van Noorden, 2013). The overall intent of the survey was to understand 1) how researchers perceived safety, 2) 
safety compliance behaviour among researchers, 3) injury or incident experiences, and 4) provide knowledge and tools for safe lab experimentation (Schroder et.al, 2016). The survey was sent to the audiences i.e. lab researchers of the collaborators (NPG, UC and Bioraft). Most of the data was collected from the United States and United Kingdom; however, the sample also included researchers from China and Japan. The researchers were from academia (universities and colleges), industry, and government laboratories. Overall, 2360 responses were received, with the majority (68\%) being from academia and in the field of Biochemistry/Biology (44\%) and Chemistry (17\%).

Findings from the survey revealed that, while $86 \%$ of all participants indicated that their labs are safe working environments, almost half reported they have experienced lab injuries (Van Noorden, 2013). Moreover, many participants lacked sufficient training on the specific hazards or agents used for their lab work (Schroder et.al, 2016). The survey revealed that before experiments, researchers from academia, compared to industry, generally assess their work risk informally and sometimes not at all (Schroder et.al, 2016). Similarly, non-compliance with wearing required lab coats and eye protection was more common among academic lab researchers than industry lab workers (Schroder et.al, 2016). Overall, the survey concluded that safety culture is not well established in academia in contrast to industry (Schroder et.al, 2016). Therefore, there is a greater need to enhance safety culture in academic labs than other lab settings i.e. industry. In fact, the director of the National Academies Board on Chemical Sciences and Technology who stated that the findings from the survey are another piece of evidence "in a growing body of reports that point to the need to improve the culture around safety in our academic laboratories"(Van Noorden, 2013b).

Since a majority of the accident reports and studies are from the United States, it is unknown if the need to improve academic lab safety culture is also applicable to the lab researchers that operate in Canadian universities. Therefore, the goal of this study was to examine the state of safety culture among wet lab researchers from a medium-sized Canadian academic institution (University X).

\subsection{Chapter 4 objectives}

The following four objectives were examined to help achieve the goal of this chapter.

1) identify the perception and attitude University X's workers have towards lab safety

2) identify the safety practices/behaviours employed by the workers when in their lab environment

3) further understand the manner in which lab safety may be managed at the University 
4) compare the safety culture at University $X$ to that of the academic institutions that participated in the 2012 international survey.

\subsection{Methods and materials}

\subsubsection{Recruitment}

Before starting the study, ethics approval was obtained from University X's Board of Ethics. Subject recruitment was performed via emails, in person, and by posting of flyers within several buildings of the University. The subject inclusion criterion was: any member of University X (students, faculty, and staff) currently engaged in chemistry and/or biology wet lab activities within the academic institution.

\subsubsection{Questionnaire}

To obtain the data for this chapter, an online survey was administered to participants. The majority of the survey questions were adapted from the 2012 international Laboratory Safety Culture Survey, which was successfully tested for reliability and validity. In order to test for validity, a panel of Board Certified Safety Professionals, chemists, and other experts involved in academic chemistry lab safety reviewed the survey (I. Schroeder, personal communication, April 11). After rigorously reviewing the survey questions, the panel concluded that the survey instrument had face validity i.e. the questions were directly related to the research intent (I. Schroeder, personal communication, April 11). Additionally, the survey questions were tested for construct validity by evaluating pilot test results with known research lab operations at UCLA (I. Schroeder, personal communication, April 11). The responses obtained directly correlated with known safety operations at UCLA labs. Furthermore, the survey questions received a high reliability Guttmann-Cronbach alpha coefficient of 0.848 (Van Noorden, 203; R. Jordan, I. Schroeder, personal communication, April (10 and 12), 2017). This shows that the survey can be safely reproduced in other studies and achieve an accurate assessment of safety culture. In fact, this survey was used and mentioned in other lab safety studies (Mohammed et al., 2015; and Schroeder et al., 2016).

For the purposes of this study, the questionnaire (Appendix F) were divided in four sections (A - D). Part A included demographic data such as age, lab work experience, gender, job title, etc. Part B collected data on workers and students' perceptions and attitudes towards lab safety. The focus in Part C was on workers and students' lab practices and behaviours. Finally, the questions in Part D focused on the management of lab safety from the perspective of those in supervisory roles such as supervisors, Principal Investigators (PIs), lab managers, technicians, etc. For this specific study, the questionnaires were administered online via Survey-Monkey. The 
majority of the questions were written in a multiple-choice format, in which a list of responses was provided for respondents to choose from. To identify the difference in safety culture between the lab personnel from University X and other universities, a comparison of common survey questions from this study and the 2012 international study was conducted. Since the overall purpose of this chapter was to obtain an understanding of the safety culture among University X's lab personnel, all collected data were descriptively analyzed and depicted in frequency distribution tables and graphs.

\subsection{Results}

\subsubsection{Participant characteristics}

Out of approximately 159 lab personnel approached, 61 individuals consented to participate in this study. However, since five declined from participating, a total response rate of 35\% (56/159) was obtained. As indicated in Table 4.1, the survey participants for this study mostly came from University X's Chemistry and Biology study fields, with a participation rate of $84 \%$ while other programs only made up $16 \%$ of the participants. It should be noted that graduate male students (63\%) between 18-30 years of age (66\%) made up most of the survey participants. Regarding lab work experience, the majority (57\%) of the participants said they have worked in a lab setting for at least one year. It was also indicated by $83 \%$ of the participants that 1 to 10 people work in their lab(s).

\subsubsection{Perception and attitude towards lab safety}

According to most participants (96\%), lab safety should be the concern of all stakeholders (i.e. lab supervisors/managers, faculty, technicians, and students). In fact, $73 \%$ of the participants reported that safety is 'very' important to them personally. However, $4 \%$ chemistry and biology participants agreed that safety rules and regulations negatively affect their lab productivity (Table 4.2). Similarly, $66 \%$ of all participants indicated that lab safety is 'slightly' to not at all impacted by workplace inspections. Furthermore, $16 \%$ of the participants (14\% from the Chemistry and Biology programs, and $2 \%$ from other study fields) indicated that they feel that the safety procedures in their labs "should be a little more stringent".

\subsubsection{Laboratory safety management}

Table $4.3 \mathrm{a}$ indicates that $48 \%$ of participants agreed that safety in their labs takes precedence over all other lab priorities. However, $39 \%$ of participants revealed that the overall safety in their labs could be improved. In support of this, $82 \%(14 / 17)$ of the participants holding supervisory roles listed various barriers (e.g. time and hassle, competing priorities, untrained staff, inadequate equipment, etc.) that they believe are preventing safety 
improvement in their labs. While the OHSA requires supervisors to ensure that workers are performing their task(s) safely, $12 \%$ of the participants who hold supervisory roles revealed that no one in their labs is assigned the primary responsibility for ensuring compliance with safety policies and procedures (Table 4.3b). While 90\% of the participants expressed that their lab always has sufficient Proper Protective Equipment (PPE) necessary for their work, 35\% rated their lab's PPE, Safety Data Sheets (SDSs), and eye wash stations as 'somewhat easily accessible (Table 4.3a).

When asked to indicate who has provided them with lab safety training, $5 \%$ of the participants indicated that they have never received any such training. Furthermore, $11 \%$ of the participants indicated that they are not aware of the minimum safety training required for their particular lab activity, and have not been trained on the specific hazards/agents they work with. Additionally, some participants (9\%), predominantly from chemists and biologists, expressed that they are unaware of what to do in case of lab fires, spills, and other emergencies. Although $94 \%$ of the participants who hold supervisory roles indicated that appropriate safety measures have been taken to prevent accidents and injuries in their lab (Table 4.3b), 15\% of all participants revealed that, at least once, they have sustained lab injuries (Table 4.3a). They varied from chemical inhalation to needle stick injuries (Figure 4.1). Also worth noting is that $35 \%$ of the participants who hold supervisory roles reported that they are aware of at least one major injury that has occurred in their labs within the past 12 months (Table $4.3 \mathrm{~b}$ ).

\subsubsection{Laboratory practices/behaviours and incidents}

Despite the fact that many of the lab substances are high-risk chemicals, and that many hours are spent weekly to handle them, $27 \%$ of the survey participants indicated that do not carry out any form of risk assessment before performing lab experiments (Table 4.4). Even among the participants who carry out risk assessment, 49\% revealed that their risk assessment is performed informally.

Table 4.4 indicates that working alone is an every-day occurrence in the labs of $42 \%$ of the participants. In fact, $12 \%$ of respondents revealed that they do not receive any regular check-up from their supervisors to ensure they are safely performing their experiments.

Although $98 \%$ of the study participants indicated they can appropriately use the PPE required for their lab work (Table 4.3a), only 40\% expressed they wear their PPE at all times when working (Table 4.4).

Regarding incident reporting, $38 \%$ of the participants who sustained lab injuries revealed that, at least once, they did not report their injuries to their supervisor or principal investigator (Table 4.4). This is despite the fact that 
$94 \%$ of the participants claimed to feel comfortable speaking to their superior(s) about safety concerns regarding their lab work (Table 4.3a). Furthermore, 13\% of the participants who hold supervisory roles agreed that the number of minor injuries in their labs could have been reduced if safety procedures were always followed (Table $4.3 \mathrm{~b}$ ).

\subsubsection{Safety culture: a comparison between University $X$ and other universities}

When comparing findings from this study to that of the 2012 international lab safety culture survey, numerous similarities and differences were identified (Figure 4.2). For example, a similar percentage of respondents in both studies, University X (91\%) and the academic researchers from the 2012 international survey (86\%), agreed that their labs are safe places to work. Both sets of respondents listed various elements that they believe are the barriers to safety improvement in their labs. Some of the common barriers include lack of or inadequate safety training, competing priorities, lack of funds for safety equipment, etc. (Figure 4.3). Working alone was a common issue among the participants from both studies (42\% University X participants, and 32\% participants from the 2012 study). Additionally, informal assessment of risk before lab experiment is common among the participants from both studies (49\% University X, and 57\% participants from the 2012 study). In terms of differences, $27 \%$ of the University X participants compared to only $12 \%$ of the 2012 study participants indicated they do not perform any risk assessment before lab experiments. Additionally, $38 \%$ of the participants from this study, compared to $26 \%$ from the 2012 study, said that they have sustained lab injuries and did not report them to their supervisor.

\subsection{Discussion}

This study was conducted to identify the safety culture that currently exists among the lab personnel of University X (a Canadian medium-sized university). The ultimate purpose was to determine if there is a need for safety culture improvement within the wet labs of this institution, as is the case in other universities.

The findings revealed that there may be inconsistencies between the importance placed on safety and the attitude adopted towards safety at University X. Specifically, although an overwhelming majority of the participants stated that safety is of high importance to them, their view on certain lab safety elements showed otherwise. For example, inspections were viewed by many participants as having little to no significant impact on lab safety.

Inspections, as previously mentioned (Chapter 2), are necessary to identify safety deficiencies and provide corrective actions for an overall healthier and safer lab environment (Foster, 2003; and Government of Ontario, 2014). Therefore, with this negative view on inspections, one fears that safety checks may not even be conducted in some of the labs, as was suggested in the findings from Chapter 2 of this research. Additionally, the fact that $16 \%$ of 
the participants said that their lab's safety protocols are not stringent, may indicate that the safety protocols established for the labs perhaps do not make inspections mandatory, as they ought to be (Government of Canada, 2016). However, even if the safety checks were made mandatory and performed, one still fears that when deficiencies are identified they may not be addressed. In support of this, many safety deficiencies identified from the 2012-2014 safety reports reappeared in the Chapters 1 (inventory) and 2 (inspection) findings of this research thesis.

However, it was reassuring to find most of the survey participants express that safety should be the concern of all lab stakeholders. This shows that there is a common interest in and care for lab safety among workers and researchers. However, the various gaps identified suggest that safety management is currently not at its best within some of the labs at University X.

For example, while section 27 of the OHSA requires supervisors to ensure that workers safely carry out their respective tasks, many of the participants indicated that their supervisor does not check on them to ensure they are properly carrying out their lab work. In fact, many of the participants who hold supervisory roles revealed that no one in their lab is assigned the responsibility for ensuring compliance with safety rules. This not only shows noncompliance with OHSA requirement, but it also indicates that some of the researchers at this University may lack awareness of their OHSA obligations as supervisors. This finding also suggests that enforcement of safety rules may be lacking within the labs. As a result, workers may become too lax in their lab practices/behaviours. In fact, many (20\%) of the participants revealed that they do not always wear their PPE during lab operations. This also shows possible non-compliance with section 28 of the OHSA, which asks workers to "use or wear the equipment, protective device, or clothing required" for the safe performance of their tasks. Additionally, many participants indicated that they have sustained injuries which they have not reported to their supervisors. This lack of incident reporting shows that they are not doing their part as per the IRS. It also may hinder lab supervisors from complying with section 52 of the OHSA, which requires them to report in writing, to health and safety representative(s) or committees (e.g. the University's Joint Health and Safety Committee), about any accident or near-miss incidents that may have prevented worker from performing their task. Furthermore, before lab experiments, a risk assessment is informally conducted by $49 \%$ of the survey participants. In fact, $27 \%$ of the participants indicated that no form of risk assessment is performed before lab experiments. Also, working alone is a frequent occurrence within the labs at this academic institution. 
As part of a good occupational health and safety management plan, risk assessments are crucial when planning a lab experiment (Canadian Center for Occupational Health and Safety, 2009). For one, they help identify the hazards and potential risks involved in the experiments. Secondly, assessing risk before conducting experiments helps identify how to safely undertake lab operations (US National Research Council, 2011c). When risk is formally assessed (i.e. using an organization's approved form etc.), there is less subjectivity in the process, and underestimation of the dangers (actual and potential) that could lead to accidents is minimized (Schroeder et.al, 2016). The fact that many of the lab personnel from University $X$ informally assess risk before experiments, suggest that their understanding of the dangers associated with their lab work may not be entirely accurate. This may explain why most survey participants have a low perception of the risk associated with their lab work, despite the numerous hazardous chemicals present in their labs and in poor labeling and storage conditions (refer to Chapter 1).

While it is common among most university researchers (Van Noorden, 2011), working alone increases the risks associated with lab activities, making it much easier for injuries to occur and for emergency response(s) to be delayed in case of accidents (University of Sussex, 2012; and Health and Safety Authority, 2017.). In fact, one of the contributing factors suggested for the 2011 fatal lab accident at Yale University is that the victim was alone while working on her experiment (Van Noorden, 2011).

In general, when lab safety rules are not enforced, workers may become too lenient about safely conducting their tasks. As a result, this may facilitate workers' exposure to the hazards (actual and/or potential) associated with their lab operations. Workers' exposure to hazards and risky conditions can also increase when safety devices and equipment necessary for lab operations are not made available or easily accessible, as well as when safety education is not provided through training. Additional findings from this study suggest that workers and students may be at risk of exposure to the hazards and risky conditions present in their labs. This is because: 1$)$ some (10\%) of the participants said that their labs are without sufficient PPE; 2) even among the labs with PPE, this safety equipment along with SDSs, eyewash stations, etc., are only somewhat accessible according to 35\% of the participants; and 3) safety training is not provided to all lab workers. These findings show non-compliance with sections 25 to 27 of the OHSA, which require that workers be: 1) informed about the hazards and risks associated with their task(s), 2) provided with all the protective equipment or devices necessary to safely perform their task(s), and 3$)$ that they be instructed or trained on how to safely carry out their lab task(s) (Government of Ontario, 2014a; and Government of Ontario, 2014b). Also, the fact that there are still some participants who are not aware of what to do in case of a lab 
emergency such as fire, spills, etc., shows non-compliance with section 130 of the Industrial Establishments Regulations (R.R.O.1990, Reg,851), which requires that workers be "trained in the proper use of emergency measures and procedures" (Government of Ontario, 2016).

The safety gaps identified from the findings of this study not only imply that safety may not be well prioritized within some of the labs at University $X$, but that they may contribute to the hindrance of safety improvement within the labs. Therefore, there is an overall need to enhance the current culture of safety within the labs and among workers. In support of this, $82 \%$ of the participants who hold supervisory roles listed various barriers that they believe are currently preventing safety improvements in their labs. Additionally, the need to enhance the safety culture in University X's wet labs was further confirmed when some of the results in this study were compared to that of the 2012 international lab safety culture survey. For instance, even though working alone was a common practice among both sets of study participants, a higher percentage was found at University X. Also, the lack of injury reporting was a bigger issue at University X. This is because a higher percentage of participants at this university indicated they sustained injuries that they have never reported to their lab superiors. Lastly, a greater percentage of workers who do not assess risk before lab operations was found among the participants from University X. Therefore, similar to labs in most of today's academic institutions, researchers and students from University $\mathrm{X}$ will benefit from the fostering of a stronger and more positive safety culture in their laboratories

\subsection{Study limitations}

The possibility for response bias is one of the limitations in this study. Response bias may have caused participants to provide responses that portray them as safety oriented individuals. Nonetheless, the findings obtained from this study still provide a general understanding of the state of safety culture among some of the University's lab personnel.

\subsection{Chapter conclusion}

To determine if there is a need for lab safety culture improvement within University $\mathrm{X}$, this study investigated the current state of safety culture at the University. The findings revealed that many of the participants have a negative attitude towards certain safety elements (e.g. safety rules and inspections). This suggests that safety may not be prioritized within the labs. Specifically, even though a majority of the participants expressed a high esteem for safety, numerous gaps were identified in their lab safety behaviours/practices and in the manner in which safety is managed within their labs. For instance, lab safety training was not provided to all workers; some labs still 
lacked PPE; and some labs had no individual assigned the responsibility to ensure experiments are safely carried out. Furthermore, many lab personnel indicated they do not perform any form of risk assessment before experiments. Additionally, many of the workers whose labs have sufficient PPE said they do not always wear their protective coats when working. Also, working alone is a very common behaviour among many of the lab personnel. These findings suggest non-compliance with numerous OHSA requirements and general lab safety best practices. Thus, there is a need to enhance the safety culture that currently exists within the University's labs and among the lab workers. 


\begin{tabular}{|c|c|c|}
\hline Categories & Response Options & Participants \\
\hline Gender & $\begin{array}{l}\text { Female } \\
\text { Male }\end{array}$ & $\begin{array}{l}37 \% \\
63 \%\end{array}$ \\
\hline Age & $\begin{array}{c}\text { Under } 18 \text { years } \\
18-30 \text { years } \\
31-50 \text { years } \\
51-60 \text { years } \\
\end{array}$ & $\begin{array}{c}2 \% \\
66 \% \\
23 \% \\
9 \% \\
\end{array}$ \\
\hline Current Position & $\begin{array}{c}\text { Research Assistant } \\
\text { Graduate Student } \\
\text { Supervisor/manager } \\
\text { Technician } \\
\text { Faculty } \\
\text { Other } \\
\end{array}$ & $\begin{array}{c}7 \% \\
50 \% \\
4 \% \\
5 \% \\
16 \\
18 \% \\
\end{array}$ \\
\hline Lab type & $\begin{array}{c}\text { Wet lab } \\
\text { Dry } \\
\text { Both }\end{array}$ & $\begin{array}{l}48 \% \\
4 \% \\
48 \% \\
\end{array}$ \\
\hline Department & $\begin{array}{c}\text { Chemistry } \\
\text { Biology } \\
\text { Other }\end{array}$ & $\begin{array}{l}21 \% \\
63 \% \\
16 \%\end{array}$ \\
\hline Number of worker & $\begin{array}{c}1-10 \text { staff } \\
11-20 \text { staff } \\
21-100 \text { staff }\end{array}$ & $\begin{array}{l}82 \% \\
16 \% \\
2 \%\end{array}$ \\
\hline Time spent in current lab & $\begin{array}{l}\text { Less than } 5 \text { months } \\
5-11 \text { months } \\
1-10 \text { years } \\
11+\text { years }\end{array}$ & $\begin{array}{c}25 \% \\
12 \% \\
54 \% \\
9 \%\end{array}$ \\
\hline Time spent in lab in general & $\begin{array}{l}\text { Less than } 5 \text { months } \\
5-11 \text { months } \\
1-10 \text { years } \\
11+\text { years }\end{array}$ & $\begin{array}{l}9 \% \\
7 \% \\
57 \% \\
27 \% \\
\end{array}$ \\
\hline
\end{tabular}




\begin{tabular}{|c|c|}
\hline \multicolumn{2}{|c|}{ Table 4.2: Perception and attitudes towards safety among University X's lab researchers and students } \\
\hline Survey items and response options & Participants \\
\hline Importance placed on lab safety & \\
Safety is very important & $73 \%$ \\
Safety is quiet important & $27 \%$ \\
\hline Lab safety rules and regulations negatively impact lab & \\
productivity & \\
Agree & \\
Disagree & $4 \%$ \\
Neither agree nor disagree & $67 \%$ \\
Safion & $29 \%$ \\
Impact of inspection of lab safety & \\
Safety is greatly improved by inspections & \\
Safety is slightly improved by inspections & \\
Inspections have no significant impact on safety & $16 \%$ \\
Safety is slightly compromised by inspections & $50 \%$ \\
Don't know & $2 \%$ \\
& \\
\hline
\end{tabular}




\section{Table 4.3a: Lab safety management based on responses from all participants}

\section{Survey items and response options}

\section{Participants}

I feel comfortable speaking to my supervisor or principal investigator about safety concerns regarding my own lab work

\begin{tabular}{|c|c|}
\hline $\begin{array}{c}\text { Agree } \\
\text { Neither agree nor disagree } \\
\text { Don't know }\end{array}$ & $\begin{array}{l}94 \% \\
2 \% \\
4 \%\end{array}$ \\
\hline $\begin{array}{l}\text { I feel that my lab is a safe place to work } \\
\qquad \text { Agree } \\
\text { Neither agree nor disagree }\end{array}$ & $\begin{array}{c}91 \% \\
9 \%\end{array}$ \\
\hline $\begin{array}{l}\text { I feel that the safety procedures in my current lab are: } \\
\text { Too stringent } \\
\text { About right } \\
\text { Should be more stringent } \\
\text { I don't know }\end{array}$ & $\begin{array}{c}5 \% \\
75 \% \\
16 \% \\
4 \% \\
\end{array}$ \\
\hline $\begin{array}{c}\text { I am aware of and understand the minimum training requirement } \\
\text { Agree } \\
\text { Disagree }\end{array}$ & $\begin{array}{c}93 \% \\
7 \% \\
\end{array}$ \\
\hline $\begin{array}{c}\text { I received sufficient training on the specific agent/hazards I work with } \\
\text { Agree } \\
\text { Disagree } \\
\text { Neither agree nor disagree }\end{array}$ & $\begin{array}{c}86 \% \\
4 \% \\
10 \% \\
\end{array}$ \\
\hline $\begin{array}{c}\text { My lab has sufficient supplies of PPE necessary for my lab work } \\
\text { Agree } \\
\text { Disagree } \\
\text { Neither agree nor disagree }\end{array}$ & $\begin{array}{c}90 \% \\
4 \% \\
6 \%\end{array}$ \\
\hline $\begin{array}{c}\text { The safety equipment that my lab uses are always certified and in good wo } \\
\text { Agree } \\
\text { Disagree } \\
\text { Neither agree nor disagree } \\
\text { Don't know }\end{array}$ & $\begin{array}{c}76 \% \\
2 \% \\
13 \% \\
9 \%\end{array}$ \\
\hline $\begin{array}{l}\text { PPE, SDSs, eye-wash stations and other safety equipment and material are } \\
\text { Easily accessible } \\
\text { Somewhat accessible } \\
\text { Don't know }\end{array}$ & $\begin{array}{c}63 \% \\
35 \% \\
2 \%\end{array}$ \\
\hline $\begin{array}{l}\text { Safety in my lab is: } \\
\qquad \begin{array}{c}\text { Paramount and takes precedence over other lab priorities } \\
\text { Very important } \\
\text { Of equal importance to other lab priorities }\end{array}\end{array}$ & $\begin{array}{l}48 \% \\
39 \% \\
13 \%\end{array}$ \\
\hline $\begin{array}{c}\text { I am aware of what to do in case of fires, spills etc. At my current lab? } \\
\qquad \text { Agree } \\
\text { Disagree }\end{array}$ & $\begin{array}{c}91 \% \\
9 \% \\
\end{array}$ \\
\hline $\begin{array}{l}\text { At my current lab, I have sustained injuries of various kinds } \\
\text { Agree (on more than one occasion) } \\
\text { Disagree }\end{array}$ & $\begin{array}{l}15 \% \\
85 \%\end{array}$ \\
\hline $\begin{array}{l}\text { Overall, the safe in my lab could be improved } \\
\text { Agree } \\
\text { Disagree } \\
\text { Neither agree nor disagree }\end{array}$ & $\begin{array}{c}39 \% \\
7 \% \\
54 \%\end{array}$ \\
\hline
\end{tabular}




\section{Table 4.3b: Lab safety management based on responses from lab superiors}

\section{Survey items and response options}

Who in your lab is responsible for ensuring compliance to lab safety

requirements?

Lab manager or senior technician Principal investigator or head-supervisor A graduate student

The lab safety staff from University X's health and safety department No one has been assigned or specifically assumed this responsibility

Have appropriate measures been taken in your lab to protect employees from injury?

\section{Yes Don't know}

Are you aware of any major injuries that occurred within the past 12 months at your lab?

$$
\begin{aligned}
& \text { Yes } \\
& \text { No }
\end{aligned}
$$

Are you aware of any major injuries that occurred within the past 12 months at your lab?

$$
\begin{aligned}
& \text { Yes } \\
& \text { No }
\end{aligned}
$$

The number of minor injuries could have been reduced if safe lab practices were always followed

$$
\text { Agree }
$$$$
\text { Disagree }
$$

Neither agree nor disagree

\section{Participants}

$35 \%$

$29 \%$

$18 \%$

$6 \%$

$12 \%$

$94 \%$

$6 \%$

$6 \%$

$94 \%$

$35 \%$

$65 \%$

$13 \%$

$27 \%$

$40 \%$

$20 \%$ 


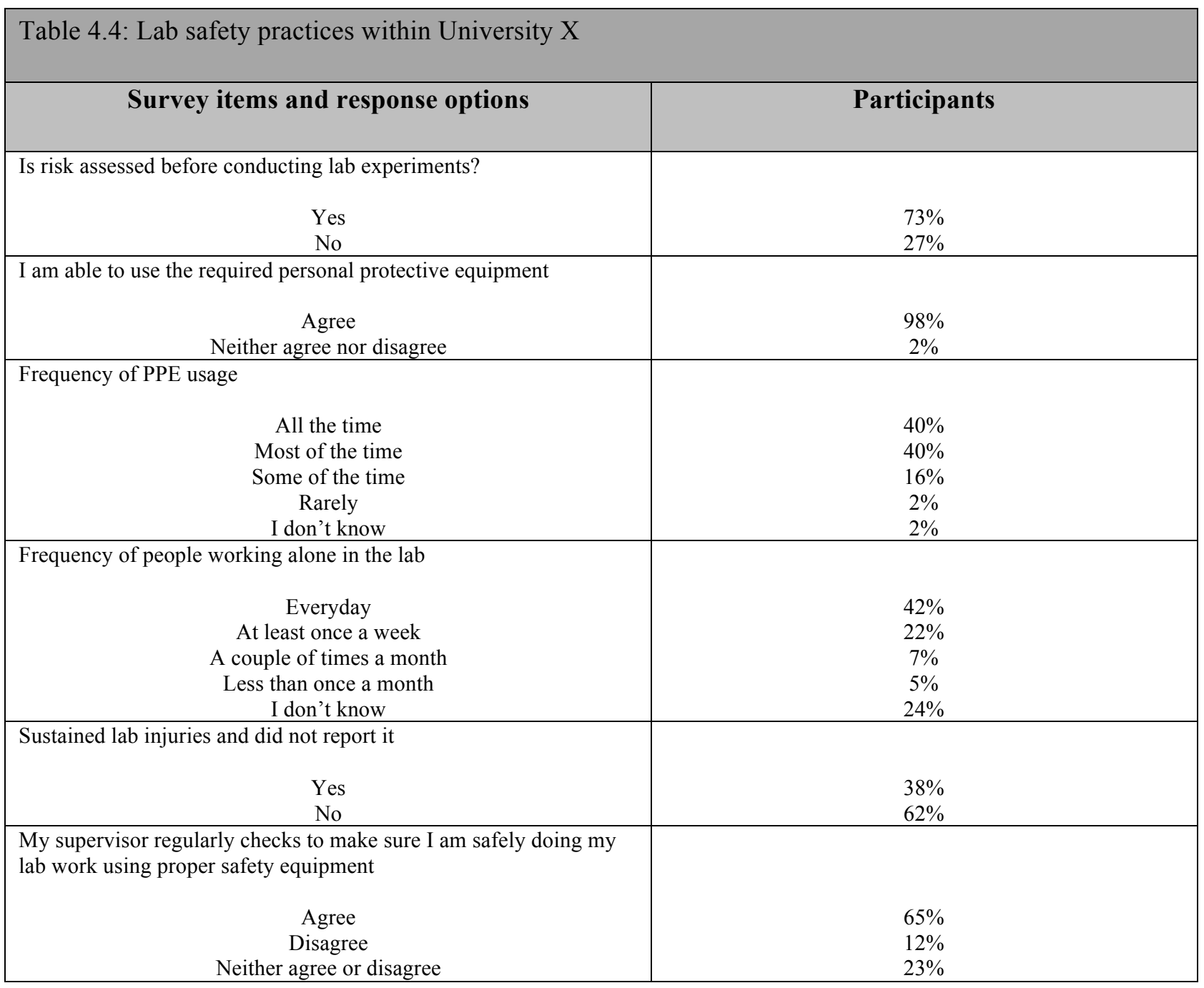


Figure 4.1 What was the nature of your injury or injuries? Check all that apply

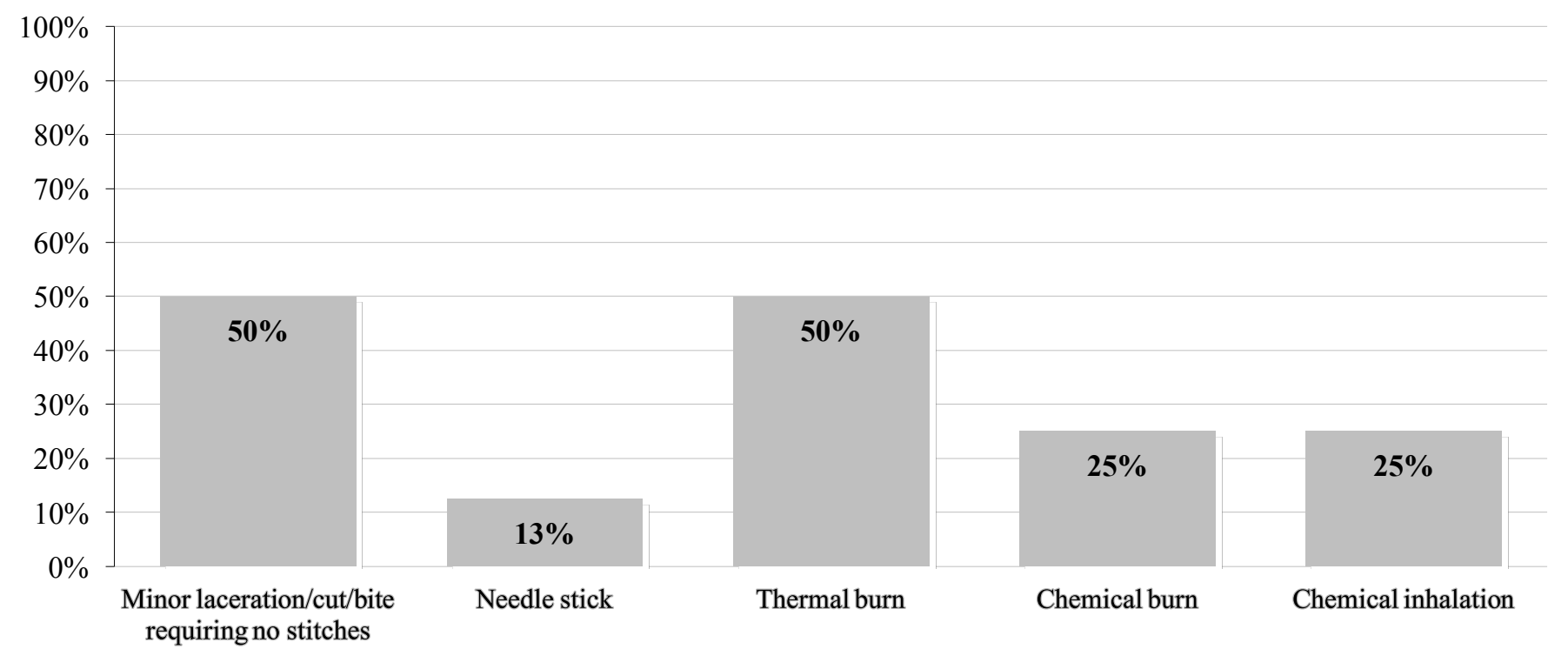

Note that the values do not equal $100 \%$ as an individual may have indicated that they have experienced more than one injury 
Figure 4.2 Lab safety improvement barriers: medium-sized Canadian university vs 2012 study participants

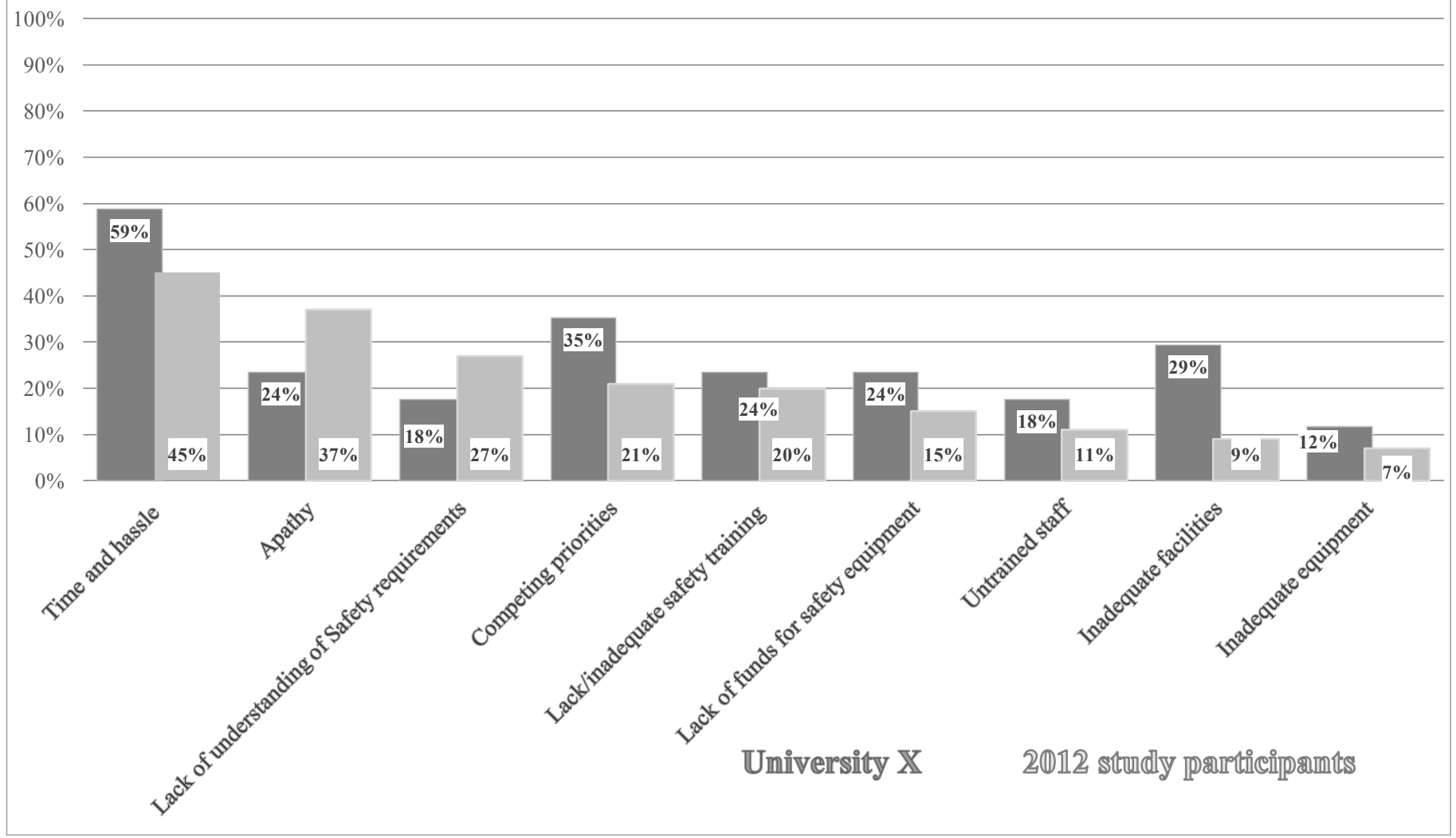


Figure 4.3 Lab safety culture: medium-sized Canadian University vs other 2012 study participants

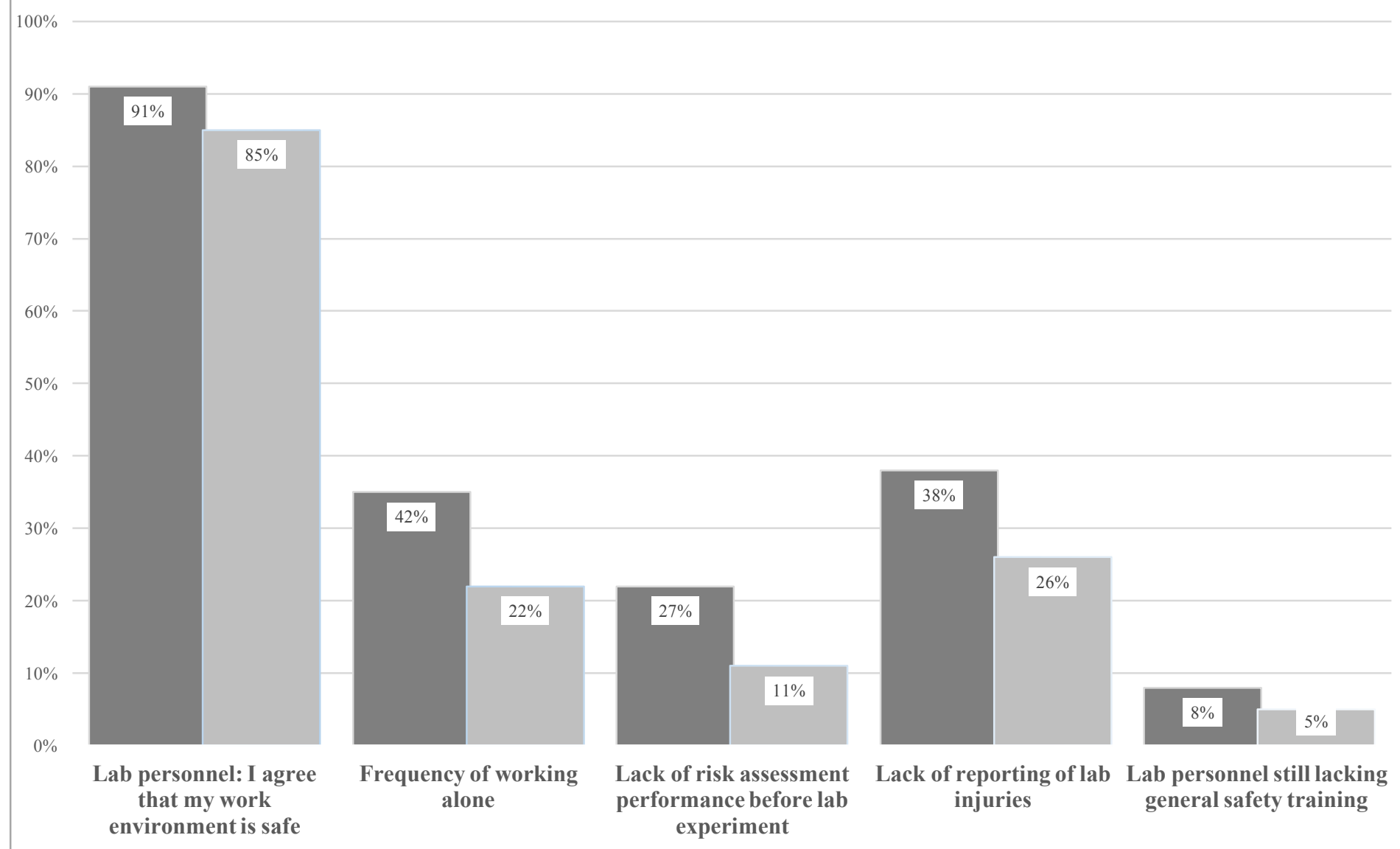

University X 2012 studely pæritieip@mits 


\section{Chapter 5- Conclusion and Recommendations}

\section{Research Overview}

As alluded to in the previous chapters, the potential for exposure to a wide range of hazardous chemical and other substances, which present acute and/or chronic health risks, makes academic labs unique research and teaching environments (Gutiérrez et al., 2013; Adane \& Abeje, 2012; and Langerman, 2009). Over the past few years, reports of accidents in university laboratories have increased steadily. As a result, the current state of safety in university wet lab has been questioned by academia and industry alike (Bell \& Fogler, 2001; Jia, 2016; and US National Research Council, 2014). Additionally, various studies have revealed many deficiencies or gaps in the way safety is managed, practiced, and perceived within academic institution lab settings (Haberman, 2014; Stanford University, 2014; and Engida, 2011). These have led many safety professionals and academics to conclude that wet laboratories in academic institutions lack a strong and positive safety culture (American Chemical Society, 2012; US National Research Council, 2014; McGarry et al., 2013; Van Noorden, 2013; and Benderly, 2016). Though it is not the norm in most university settings, some countries, especially the United Kingdom, use the threat of legal action as an incentive to help enhance lab safety within universities (Van Noorden, 2011).

While the recent accidents and study findings predominantly reflect U.S. laboratories, there is a lack of literature on the state of safety within the wet labs of Canadian universities. Therefore, this research examined the state of safety that currently exists within the wet laboratories of a medium-sized Canadian academic institution (University X). The overall goal of this research study was to obtain a descriptive understanding of how safety is perceived, managed, and practiced at University X. This was performed to determine if 1) lab workers at University $\mathrm{X}$ face any health and safety risks when in their labs, and 2) there is a need to improve the safety culture in the labs, as is the case in most U.S. universities.

\subsection{Study objectives and methods}

To achieve the goals of this research, the following four objectives were examined:

1) Understand about the chemicals handled in the labs, to get an idea of the associated hazards and risks

2) Identify the safety practices/behaviors employed by workers when in their labs

3) Ascertain the manner in which safety may be managed in the labs

4) Identify the overall culture of safety that exists among lab personnel 
To achieve objective \#1, chemical inventories were carried out in all participating labs. For objectives \# 2 an online safety culture survey was administered to all participants. Objective \#3 was achieved by performing safety inspections in all participating labs. Lastly, objective \# 4 was also achieved by administering an online safety culture survey to all participants.

\subsection{Key research findings}

\subsubsection{Overall research findings}

The overall findings from this research thesis suggest that there are numerous hazardous chemicals present within the 21 labs examined. Many participants revealed that they spend long hours every week handling their lab chemicals. The numerous instances of non-compliance with OHSA requirements and general lab best practices identified in this study, suggest that safety may not be well prioritized within the labs and among workers. Therefore, similar to other universities, the safety conditions and culture at University $\mathrm{X}$ are also in need of improvement. Below is a summary of the results that led to the above conclusions.

\subsubsection{Hazardous chemicals within some wet labs at University X (Chapter 1)}

The inventories showed that there are over 1000 chemicals present in only 21 wet laboratories at the university examined. Important to note is that many of these chemicals are high-risk, as they fall in the WHMIS signal word category of "danger" and are associated with various hazard classes e.g. flammable, oxidizing, corrosive, compressed gas, poisonous/toxic, etc. In addition to the dangers inherently associated with the inventoried chemicals, many were found improperly labelled or not labelled at all. This shows non-compliance with WHMIS requirements, set out by the government of Canada (Canadian Centre for Occupational Health and Safety, 2016b). Also, on several occasions, chemicals of incompatible hazard classes were found stored beside each other (i.e. flammables stored with oxidizers, incompatible acids stored together, etc.). This is not only an unsafe practice, but a breach of the General Safe Storage Guidelines (Health Canada, 2009, and University of Iowa, 2012). Despite the numerous high-risk chemicals present in the chemistry and biology wet laboratories, $52 \%$ of the survey participants from these study fields indicated that the level of risk associated with their lab work is "low". Compared to the Biology participants, chemists had a higher perception of the risk associated with their lab work.

\subsubsection{Safety deficiencies found in some wet labs at University $X$ (Chapter 2)}

After inspecting 22 chemistry and biology wet laboratories, the results showed several safety violations. For one, many of the labs lacked written instructions and procedures necessary to inform workers about the proper 
practices to employ when handling hazardous substances. This is a breach of the OHSA and WHMIS, both of which require that lab workers be educated on the safe practices/behaviours to adopt when working (Government of Ontario, 2014). Secondly, many of the labs, predominantly the ones used for teaching purposes, also lacked adequate personal protective equipment (i.e. lab coats, gloves, and safety glasses), The majority (95\%) of the labs did not have stocked spill cleanup kits, despite the numerous high-risk chemicals utilized in them. Furthermore, none of the safety devices (such as safety showers, eyewash stations, chemical fume hoods, and biosafety cabinets) present in some of the labs had been inspected within the last year. This violates various standards (e.g. the American National Standards Institute-Z95, National Fire Protection Association-45, Canadian Standards Association Z316.3-95, and the National Sanitation Foundation-49), which require that these safety devices to be regularly (per year) checked (CCOHS, 2017; Public Health Agency of Canada, 2005; and Ciloski,2005). Additionally, hazardous wastes materials and chemicals were found unattended and stored in the fume hoods and biosafety cabinets of many of the labs. This is a sign of non-conformance with laboratory best practices (Creighton University, n.d.).

\subsubsection{Safety attitudes and practices among University X's lab personnel (Chapter 3)}

Despite the hazardous nature and poor labeling and storage conditions of many of the inventoried chemicals, many participants viewed the level of risk associated with their work as low. This indicates that there may be a gap between perception of risk and actual risk. Furthermore, many of the survey participants had a negative attitude towards certain safety elements. For example, some perceived inspections as insignificant to the safety improvement of their labs; and others believe that safety rules and regulations have a negative influence on their lab productivity.

Regarding safe practices, many of the participants indicated they do not perform risk assessments before lab experiments. Although many participants said their lab has sufficient PPE supplies, the PPE is not always utilized during experiments. Furthermore, working alone occurs frequently among many of the participants. Although OHSA (Government of Ontario, 2014c) requires employers to monitor and maintain accurate safety records regarding their lab activities, many of the participants holding supervisory roles reported they do not have access to their labs' safety performance and compliance records.

\subsection{Findings discussions and implication of research}

Working within University X's chemistry and biology wet laboratories potentially involves coming into contact with a wide range of dangerous chemicals. As a result, there may be a greater risk for exposure to chemical 
hazards (Eldridge, 2012). Further findings from this research identified numerous safety violations present within the labs. These deficiencies showed that there is a lack of full compliance with established laboratory safety rules (OHSA, WHMIS, etc.) and non-conformance with lab best practices within the University. This finding is consistent with previous studies of other academic laboratories (Wu, Liu, \& Lu, 2007; Haberman, 2014;Huising \& Silbey, 2011; BioRaft, Nature Publishing, \& University of California Laboratory Safety, 2012; Engida, 2011; and Simpson, 2015). In particular, Simpson (2015) found a significant number of non-compliance events present at the 480 university research laboratories she examined for her study. Chemistry and Biology were the two departments with the highest number of non-compliance events. Furthermore, Simpson (2015) also found that the departments with the highest number of non-compliance issues also had the highest rates of injuries, such as lacerations, punctures, chemical inhalation, burns, bruises, etc. This indicates that non-compliance to safety requirements results in adverse effects. This is demonstrated by the numerous wet lab accidents that have recently occurred at many universities around the world (Simpson, 2015).

One of the safety violations identified in the current study is the lack of safety training for some of the participants who handle hazardous agents. The lack of safety training is also a common problem in many other universities (Stanford University, 2014; Engida, 2011; Haberman, 2014; and BioRaft, Nature Publishing, \& University of California Laboratory Safety, 2012). However, it was even more surprising to find that some of the participants from this research were not aware of the required safety training necessary for their lab work. This could be due to 1) low attendance to lab safety training, or 2) poor communication of lab safety training to workers and students. Training is vital to the safety of the worker and the lab environment (US National Research Council, 2011b). It helps lab personnel understand the hazards and risks associated with their work, so that appropriate measures can be taken to safely carry out those lab operations (Wu et al., 2007). Therefore, it is important to ensure that safety training is well integrated and enforced within university lab operations.

The low-risk perception that this study found among many of the participants indicates a potential false sense of security among University's wet lab workers. This is because contrary to the findings from the observational portions of this research, a majority of the survey participants believed that their labs are a safe working environment. The concern with this false sense of security is that workers may be more likely to ignore proper safety procedures while conducting lab experiments. This is supported by the Health Belief Model which states that, the perception one has of danger and risk will influence their behaviour choices concerning safety (Oben, n.d.; 
Rosenstock, Strecher, \& Becker, 1988; and University of Twente, 2017). For instance, if lab personnel do not believe they are working with hazardous substances and in unsafe environments, they most likely will not act safely when handling those substances. However, if they perceive that a high risk exists, they will consciously or even unconsciously take the precautions and actions necessary to reduce their likelihood of exposure (Reniers, Ponnet, \& Kempeneers, 2014). This false sense of security may be the reason behind the unsound lab practices found among many of the participants. It was also not surprising to find many report, on more than occasion, that they have sustained injuries such as chemical inhalation, burns, lacerations, and needle stick injuries. If this false sense of security, which is also common in other universities (BioRaft, Nature Publishing, \& University of California Laboratory Safety, 2012; and Van Noorden, 2013), is not dealt with, there is a concern that more severe injuries may occur within the University's wet laboratories. As a result, it is crucial to ensure that workers are made fully aware of the hazards and risks (actual and/or potential) associated with their lab activities.

The numerous instances of non-compliance, a potentially false sense of security, and the unsound lab practices identified, suggest that a strong and positive safety culture may be lacking within the wet labs examined in this research. The findings also indicate that safety may not be given the importance or priority it deserves within the labs examined. In fact, the findings confirm Gutiérrez et al. (2013)'s statement that, “...the attitude that safety is of secondary importance (still) may exist for a significant fraction of current laboratory research personnel".

The poor safety culture that currently exists within the wet labs of universities, including University X, may be due to the organizational structure found in most of these institutions. The significant amount of research conducted independently and with generally limited administrative support and oversight from top management (e.g. university president, chairs, and deans), makes universities a unique work environment (Gutiérrez et al., 2013a). This organizational structure has weakened the authority that University officials can exercise over the lab safety behaviours and practices of independently funded faculty members (Hill \& Finster, 2013; and Benderly, 2016). Due to this independence, university researchers generally operate under the conviction of "academic freedom" including the right to carry out research, etc., without restriction of prescribed rules (Hill \& Finster, 2013; Gutiérrez et al., 2013; \& Canadian Association of University Teachers, 2011). As such, a clear understanding of who is responsible for what, regarding safety, is often lacking among university lab personnel (e.g. top management, faculty, staff, and students) (Hill \& Finster, 2013; and Gutiérrez et al., 2013). Also, the intense pressure exerted on university faculty members to increase productivity of grant-supported research may also cause safety to be overlooked in these indoor 
work environments (Benderly, 2016). Due to this overall complex work environment, safety initiatives among faculty and staff are sometimes inconsistent and inadequate (National Research Council (US) Committee on Prudent Practices in the Laboratory, 2011b; and Hill \& Finster, 2013).

Unlike universities, industry generally treats safety as "equal importance to productivity, quality, profitability, and efficiency" (US National Research Council, 2011b). In fact, inadequate attention paid to safety in an industry lab can result in the dismissal of an employee regardless if they hold supervisory roles or not (Benderly, 2016). Furthermore, the strong corporate structure and discipline for developing and maintaining well-organized safety programs within industry labs enables a culture of safety to be respected, completely understood, and enforced from the highest level of management down in these environments (US National Research Council, 2011c). As a result of the strong lines of authority that exist in industry lab settings, new employees from academic research labs are generally surprised to find detailed planning and extensive safety checks required before running any experiments in industry labs (US National Research Council, 2011c). With the weak lines of lab safety authority that exists in the university setting, it becomes a challenge to build a strong and positive culture of safety within these environments (Gutiérrez et al., 2013a).

\subsection{Study limitations, strengths, and next steps}

\subsubsection{Study limitations and strengths}

Although this research adds to academic lab safety literature, it has several limitations. For instance, while there are multiple academic universities that facilitate wet lab activities in Canada, this research only examined one. Furthermore, although various programs from this university were asked to take part, only the biologists and chemists agreed to have many of their wet labs participate in the inventory and safety inspection portion of this research. The absence of other eligible departments and universities limited the sample size of this research, making it difficult to perform more inferential data analysis. Although inferential statistical analysis could have further enriched and solidify the findings from this research, it was outside the scope of this thesis. Nonetheless, the findings still give a descriptive understanding of how safety is perceived and managed by the members of the University that participated in the study. Therefore, the results obtained are only reflective of the laboratories examined. Also, the conclusions made in any of the thesis chapters are also only reflective of the labs that were examined and do not necessarily apply to other wet labs at this or other Canadian universities. 
Nonetheless, the findings from this research should prompt other departments from this academic institution as well as other Canadian academic institutions to examine the safety conditions and state of safety culture within their labs. Additionally, the methods utilized in this study provide a baseline from which those departments and academic institutions can investigate the safety of their lab environments.

Since this research was a cross-sectional study, the safety gaps and deficiencies identified only speak to a point-in-time and may not be reflective of working conditions on other days or seasons i.e. summer. Nonetheless, the results still give an indication of the labs' safety conditions and culture at the time of the study.

Furthermore, some of the inspection and inventory data could have been subjectively reported. This is because what might be interpreted as safe for one person may be deemed unsafe for someone else. However, the training and supervision provided to the research team helped minimize the impact of this limitation.

Response bias to the survey may have also limited the study as it is possible for participants' answers to have been influenced by the desire to be viewed as safety minded individuals. As a result, their responses may not have been entirely reflective of the actual safety conditions in the labs. However, the responses still gave a descriptive indication of how safety might be perceived and managed at the University. Also, the observational data collected (e.g. inventories and safety inspections) made it possible to validate and strengthen many of the survey responses. In fact, these observational data were instrumental in helping to clearly identify many of the safety gaps found and alluded to by the survey results.

\subsubsection{Future Directions (Next Steps)}

Apart from this research, the author is not aware of any other study that has examined the state of safety within the wet laboratories of any Canadian university. Future studies should investigate the state of safety within the labs of other Canadian academic institutions, i.e. universities and colleges. While the labs in those institutions may not be at imminent danger or risk, examining the state of safety in them may help enhance their safety conditions and practices.

In this current study, lab participation was obtained from only one department (Chemistry and Biology); therefore, future studies should utilize more recruitment methods, including social media such as Facebook, Twitter, Instagram, etc., to increase the participation from other eligible study fields. Additionally, since this research relied heavily on descriptive statistics, future studies will greatly benefit from incorporating more inferential analysis to solidify the meaning of obtained results and make conclusions that are statistically significant. 
Surveys are useful research tools; however, when reinforced with observational data collection methods, stronger and much more accurate results are obtained. Therefore, future studies should include as many observational study components as possible (e.g. lab practice observations, safety audits, focus groups, face-to face interviews, workshops, walkthroughs, etc.)

Studies have concluded that for safety in any workplace environment to be efficient and effective, individuals at any management level (top-down) must show commitment and involvement in safety matters (Wu, Chen, \& $\mathrm{Li}$, 2008; and Gallion, Samide, \& Wilson, 2015). The author strongly recommends that future studies focus on ascertaining the perceptions and attitudes of academic institutions' top management staff towards lab safety, as well as identifying their current behaviours/practices when it comes to lab safety management.

Since government authorities (such as the Ministry of Labour) help enforce health and safety laws i.e. ensure safety rules are being adhered to, it would be beneficial for future studies to identify the frequency of government safety visit to laboratories from academic institutions. This is because these officials are generally visible only after severe or fatal accidents occur in the work environment (i.e. 2008 UCLA chemistry lab fatality). Therefore, it would be helpful to identify how the safety conditions in the labs of today's academic institutions would be affected if these government officials were to make site visits more often i.e. before a severe or fatal lab accident. Specially, it would be beneficial to find if their presence will 1) compel and encourage academic institution top management to increase their commitment to lab safety and 2) help strengthen the functioning of the IRS within these labs.

Future studies should also investigate how poor lab safety management and unsafe practices/behaviours (e.g. handling toxic chemicals in a malfunctioning fume hood or on open lab bench, etc.) might be affecting the air quality of the lab and its building, and, in turn, workers' and/or occupants' health.

\subsection{Conclusions}

This research revealed that, working within some of the chemistry and biology wet labs from University X involves potentially coming into contact with a wide range of hazardous chemicals and/or conditions. Although no imminent danger were found in the labs, if the identified safety violations are not addressed, workers in the labs who come in contact with the hazardous chemicals (many of which were poorly labeled and stored) may run the risk of unsafe exposure. This may contribute to injury incidents. Some of the safety gaps identified include: 1) poor labeling and storage of many of the chemicals; 2) lack of spill kits in most labs; 3) not all participants have received safety training; 4) fume hoods, eyewash station, and safety showers may not be inspected regularly; 5) numerous 
reoccurrences of safety deficiencies; 6) many participants do not perform risk assessment before lab experiments; 7) working alone is an everyday practice for many participants; 8) a potentially false sense of security among workers about the level of risk associated with their lab work. All these gaps suggest that a strong and positive safety culture may be lacking within the labs examined and among many the survey participants, as is the case with lab workers from other universities (Schroder et.al, 2016). The above findings also suggest that the Internal Responsibility System may not be well integrated and demonstrated in the manner in which lab safety is perceived, managed and practiced at University X. Therefore, below are four key recommendations stemming from this research that can help improve the safety conditions of the labs examined in this research study.

\subsection{Recommendations}

\subsubsection{Enhance employer and superiors' awareness of their OHSA responsibilities}

One positive finding was the willingness of participants holding supervisory roles to acknowledge their responsibility for ensuring safety in their labs. This shows that lab personnel at University $\mathrm{X}$ have some understanding of the responsibility vested on them by the Occupational Health and Safety Act (OHSA). However, the numerous safety deficiencies and violations identified in this research suggest that this safety responsibility awareness is not fully demonstrated in their current management of certain lab safety elements (i.e. inspections, training, adequate equipment etc.). Therefore, individuals in top management roles (e.g. the University president, Academic Provost, Deans, Departmental Chairs, lab supervisors) may not entirely be aware of the OHSA and their assigned responsibilities as employers and supervisors under the Act. Also, since university researchers operate independently and under weak lines of authority, there are instances in which they may be unaware of their legal responsibilities, especially with regards to lab safety (Hill \& Finster, 2013). Therefore, we recommend that the University president, academic director, departmental chairs and deans, and supervisors who have charge over a lab environment at this medium-sized Canadian academic institution be made more aware of the OHSA, the regulations relevant to their lab hazards, and their specific duties and responsibilities under the Act.

Most medium to large universities generally have an Environmental Health and Safety (EHS) department (Hill \& Finster, 2013). This department acts on behalf of the employer to set out environmental health and safety policies and programs. Its ultimate purpose is to assist supervisors/managers in developing the necessary safety control measures to reduce and prevent accidents, emergencies, and health issues within the lab or other work environments (SafeOpedia, n.d.). Since University X has an EHS team, the institution's top management should 
work with this team to establish a university-wide lab safety program. This can help top management become more aware of the OHSA and its requirements, and provide them with the necessary support needed to carry out their legal health and safety duties.

Given that some participants lacked training on their specific lab hazard/agents, it is important to ensure such training courses are made available and well communicated to all lab personnel, as is required by WHMIS (Canadian Centre for Occupational Health and Safety, 2016a). Therefore, we recommend that lab Principal Investigators and technicians collaborate with the University's EHS team to ensure that all workers and students are made aware of and attend available safety training. They should also collaborate to ensure that the training content meets regulatory requirements (i.e. inform workers of the hazards present in their workplace, etc.) and is as directly relevant to the lab activity as possible (US National Research Council, 2011c). Furthermore, since the research activities undertaken by most lab researchers generally involve handling of hazardous substances, it would be beneficial to include lab safety as part of the training required when a lab researcher is hired. At University X, new faculty and staff are usually asked to take certain mandatory training (e.g. Violence and Harassment Prevention, Plagiarism Prevention, Equity, Diversity and Inclusion, etc.). Therefore, we recommend that lab safety training also be added to the list of required training. This safety training could also be included as an annual mandatory refresher for other faculty and staff members.

\subsubsection{Enhancement of compliance with lab safety rules and best practices}

Since many of the inventoried chemicals had labelling issues, lab supervisors and technicians should ensure that all the hazardous substances in their labs are labelled, and that deteriorating labels are replaced with clearly legible ones. Doing this will not only help facilitate compliance with WHMIS (Canadian Centre for Occupational Health and Safety, 2016b), but it will also help increase workers' awareness of the hazards and risks associated with their lab chemicals. Also, it will help lab personnel to develop a more accurate perception of their work risk(s).

Additionally, since many of the labs had numerous SDSs dating more 10 years, it is uncertain if chemical expiry dates are logged into the University's online inventory system. Therefore, we recommend that chemical expiry dates also be logged into the inventory system. This system should be designed so that when a chemical has expired, the lab supervisor who owns that chemical is promptly notified to dispose of the chemical safely. 
Given the lack of written safe operating instructions (i.e. safe operating procedures, lab safety manuals, etc.) in most labs, supervisors are encouraged to collaborate with the University's EHS team to develop and make available such safety documents, which is an OHSA requirement ( Ministry of Labour, 2015b).

Since the majority of the labs lacked spill clean-up kits, and not all participants were aware of what to do in case of a lab emergency, supervisors should collaborate with the University's EHS team to develop and implement a program that addresses emergency response for all the wet labs at the University. In addition to showing compliance with OHSA's requirement (Government of Ontario, 2014c), an emergency response program would equip lab personnel to prepare for potential accidents or other lab-related emergencies.

\subsubsection{An overall improvement of safety culture within the labs}

It was reassuring to find numerous safety initiatives (e.g. Laboratory safety signage, Biosafety program, Risk assessment management, etc.) in place for the University's labs. This shows that a culture of safety does exist within the University. However, the safety violations and minor injuries identified by this research suggest that more efforts should be made to enhance the University's overall lab safety culture. The fostering of a strong safety culture is easily achieved when there is high commitment, and intentional, collaborative effort by top management to promote safety and provide the support necessary to encourage safe lab operations (Foster, 2003; Hill \& Finster, 2013; Gutiérrez et al., 2013; and US National Research Council, 2011b). An enhanced commitment to safety by the University's top management staff (e.g. institution president, chairs/academic directors, deans, lab supervisors, etc.) can help improve the safety culture in the university's wet labs.

Some ways in which top management can enhance their commitment to safety include: 1) ensure adequate fiscal resources are set aside for the development and maintenance of lab safety programs; 2) support faculty participation in lab safety matters; 3) create an online safety forum to allow free communication of health and safety concerns from workers (Foster, 2003, p. 15); 4) discuss lab safety concerns at departmental and Teaching Assistant meetings (Foster, 2003, p. 15); and 5) establish an accountability system for lab safety knowledge and behaviours (Hill \& Finster, 2013). Doing all these will send a clear message to all workers that safety is a priority at the University (Hill \& Finster, 2013).

\subsubsection{An overall better-integrated IRS system within University's lab environments}

Even though the above recommendations were specifically for individuals in managerial positions, [since management is legally responsible for ensuring safety in the work environment], we also recommend that lab 
personnel who are not in supervisory roles work in compliance with established safety regulations and conformity with lab best practices, since the OHSA operates under the principle of IRS. Specifically, given that most individuals (the majority of whom were students) who participated in this research indicated that they handle their lab chemicals for long hours every week, and many of the inventoried chemicals were identified as high-risk substances, the following is recommended from them: 1) formal risk assessments to be conducted for lab work; 2) that PPE be worn when handling any of hazardous lab chemicals; 3) any unsafe lab incidents (regardless if they're near misses or actual accidents) be reported to their lab supervisors; and that 4) a buddy or other systems be developed to minimize the occurrence of working alone.

\subsection{Research study significance}

To the author's knowledge, no study has examined the current state of safety within the wet laboratories of any Canadian academic institution, and certainly not at the medium-sized university examined in this study (University X). Therefore, in addition to contributing to the literature in the lab safety field, this research is novel for the University X. The findings from this research have helped shed some light on the manner in which lab safety is managed, practiced, and perceived at the University. The recommendations made will help equip top management (the University President, Provost and Vice President, Deans, chairs, lab directors, Environmental Health and Safety Team, etc.) to implement appropriate measures for addressing identified safety deficiencies.

Furthermore, the findings from this research can help principal investigators, lab managers, technicians, and students become more aware about the safety conditions of their labs. It is hoped that, as a result of this increased safety awareness, workers and students will be prompted to think and act with more due diligence (i.e. become more careful and safety oriented) when conducting experiments in their labs. Finally, the findings from this research study can help prompt other Canadian academic institutions to examine the state of safety in their own labs, to ensure that researchers and students are not at risk of exposure to any hazards or risky conditions.

\subsection{Research study knowledge translation}

Upon request, the results of this research study will be shared with all lab supervisors, technicians, and managers. If each one of these stakeholders wants to gain a better understanding of the state of safety in their own lab(s), a summary report of the inspection and chemical inventories results for their lab(s) will be sent to them.

The findings in this research will also be disseminated to the supervisors of the labs that were examined in this research. Similarly, the findings will be sent to the Chair of Chemistry and Biology at University X, in the hope 
that the information will be shared with all members of the faculty and staff. Additionally, since the academic institution's EHS team is responsible for developing and carrying out lab safety initiatives and programs, and making use of all lab-related resources, a copy of this research will be sent to the EHS department, and a presentation of the study results will be conducted, upon request, for their directors and safety officers. The importance of this knowledge dissemination is that it can help the EHS team cater their programs and initiatives to the appropriate needs of the academic institution's labs. To help better planning efforts to address the lab safety issues, a copy of this research study will also be sent to the University's Joint Health and Safety Committee (JHSC). Additionally, a presentation of the study findings will be given at a JHSC meeting, if requested.

To help raise safety awareness among the University's lab workers, the Institution's Graduate Studies office will make this research document available online, via the Institution's Research Library database for anyone to access. Lastly, to help prompt other Canadian universities to examine the state of safety in their wet labs, the findings of this research will be submitted to peer-review journals for publication. Examples of some of the journals include: The Journal of Safety Research, Journal of Chemical Health and Safety, Journal of Laboratory Chemical Education, and the International Journal of Occupational Safety and Ergonomics. 


\section{Appendix A: Chemical Inventory Forms}

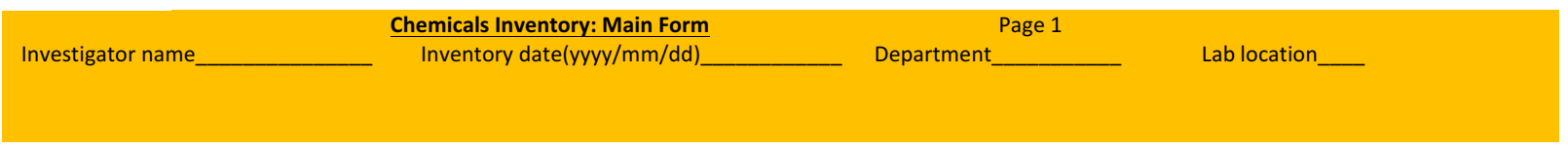

\begin{tabular}{|c|c|c|c|c|c|c|c|}
\hline Column\#1 & Column\#2 & Column\#3 & Column\#4 & Column\#5 & Column\#6 & Column\#7 & Column\#8 \\
\hline $\begin{array}{l}\text { Is chemical } \\
\text { container clearly } \\
\text { labeled? }\end{array}$ & $\begin{array}{l}\text { What kind of label is } \\
\text { on the container? }\end{array}$ & $\begin{array}{l}\text { What is the } \\
\text { chemical name as } \\
\text { per label? }\end{array}$ & $\begin{array}{l}\text { Is the container (not in use) } \\
\text { open or closed? }\end{array}$ & $\begin{array}{l}\text { Is container in good } \\
\text { condition e.g. not } \\
\text { corroded, not leaking, } \\
\text { etc.? }\end{array}$ & $\begin{array}{l}\text { Where is the } \\
\text { container } \\
\text { stored? }\end{array}$ & $\begin{array}{l}\text { Is storage area clean } \\
\text { and uncluttered? }\end{array}$ & $\begin{array}{l}\text { Is chemical } \\
\text { stored with } \\
\text { like class, e.g. } \\
\text { acids with } \\
\text { acids? }\end{array}$ \\
\hline $\begin{array}{l}\text { a) Yes } \\
\text { b) No (then } \\
\text { check 'N/A' in } \\
\text { columns } \# 2,3,4 \text {, } \\
7,8 \text {. }\end{array}$ & $\begin{array}{l}\text { a) Workplace: agent } \\
\text { name, safe handling } \\
\text { info, and reference to } \\
\text { MSDS. } \\
\text { b) Supplier: agent } \\
\text { name, supplier name, } \\
\text { the statement "refer to } \\
\text { MSDS", hazard } \\
\text { symbol(s), risk } \\
\text { phrases, precautionary } \\
\text { measures, first aid } \\
\text { measures } \\
\text { c) Other (specify): d) } \\
\text { N/A }\end{array}$ & $\begin{array}{l}\text { a) Chemical name: } \\
\text { b)N/A }\end{array}$ & $\begin{array}{l}\text { a) Open, and... I) holding } \\
\text { liquid chemical solution } \\
\text { II) holding solid or powder } \\
\text { chemical III) empty-not } \\
\text { holding any chemical } \\
\text { b) Closed, and.... I) holding } \\
\text { liquid chemical solution II) } \\
\text { holding solid or powder } \\
\text { chemical } \\
\text { III) empty-not holding any } \\
\text { chemical } \\
\text { c)N/A }\end{array}$ & $\begin{array}{l}\text { a) Yes b) No (then } \\
\text { state the condition in } \\
\text { comment section) } \\
\text { c)N/A Comments: }\end{array}$ & $\begin{array}{l}\text { a) Benchtop } \\
\text { b)Shelf c)Fu } \\
\text { me hood } \\
\text { d)Floor } \\
\text { e)Cabinet } \\
\text { f)Other } \\
\text { (specify) } \\
\text { g)N/A }\end{array}$ & $\begin{array}{l}\text { a) Yes, it's cleaned } \\
\text { and uncluttered } \\
\text { b)No, it's clean but } \\
\text { cluttered } \\
\text { c) No, it's uncluttered } \\
\text { but not clean } \\
\text { d) No, it's neither } \\
\text { clean nor uncluttered } \\
\text { e)N/A }\end{array}$ & $\begin{array}{l}\text { a) Yes b) No } \\
\text { (then comment } \\
\text { on the class } \\
\text { of chemicals } \\
\text { it's stored } \\
\text { with) } \\
\text { c)N/A Comm } \\
\text { ents: }\end{array}$ \\
\hline
\end{tabular}

\section{Appendix B: Compressed Gas Cylinders Form}

\begin{tabular}{|c|c|c|c|}
\hline & Chemicals In & essed Gas Form & Page 1 of 2 \\
\hline Investigator name & Inventory date(yyyy/mm/dd) & Department & Lab location \\
\hline
\end{tabular}

\begin{tabular}{|c|c|c|c|c|c|c|c|}
\hline Column\#1 & Column\#2 & Column\#3 & Column\#4 & Column\#5 & Column\#6 & Column\#7 & Column\#8 \\
\hline $\begin{array}{c}\text { Does the cylinder } \\
\text { have a legible status } \\
\text { tag? }\end{array}$ & $\begin{array}{l}\text { What is the } \\
\text { cylinder status as } \\
\text { per cylinder tag? }\end{array}$ & $\begin{array}{c}\text { Does the cylinder } \\
\text { have a legible } \\
\text { label? }\end{array}$ & $\begin{array}{c}\text { What is the gas } \\
\text { name as per cylinder } \\
\text { label? }\end{array}$ & $\begin{array}{c}\text { Does the } \\
\text { cylinder } \\
\text { have a cap } \\
\text { on its valve? }\end{array}$ & $\begin{array}{l}\text { Is the gas stored in } \\
\text { good condition, e.g. } \\
\text { cylinder is free of } \\
\text { corrosion, leaks, and } \\
\text { other damages, etc.? }\end{array}$ & $\begin{array}{l}\text { Is the cylinder stored in the } \\
\text { same area as other gas of } \\
\text { similar hazard class, e.g. all } \\
\text { flammable gas cylinders } \\
\text { are stored together? }\end{array}$ & $\begin{array}{l}\text { Is the cylinder stored in } \\
\text { the following manner: } \\
\text { Small/medium } \\
\text { cylinders( }(1-3 \mathrm{ft}) \text { : } \\
\text { upright in a box or crate } \\
\text { that is large enough to } \\
\text { prevent cylinders from } \\
\text { falling out } \\
\text { Tall/long cylinders }(3 \mathrm{ft} \\
\text { and more): secured in } \\
\text { an upright position to a } \\
\text { wall by a cylinder } \\
\text { stand, clamp, or chain } \\
\text { at a point } \\
\text { approximately } 2 / 3 \text { of } \\
\text { the height of the } \\
\text { cylinder }\end{array}$ \\
\hline $\begin{array}{l}\text { a) Yes } \\
\text { b) No, (than check } \\
\text { ' } N / A \text { ' in column \#2) }\end{array}$ & $\begin{array}{l}\text { a) Full } \\
\text { b) In use } \\
\text { c) Empty } \\
\text { d)N/A }\end{array}$ & $\begin{array}{l}\text { a) Yes } \\
\text { b) No (than check } \\
\text { ' } N / A \text { ' in columns } \\
\# 4,7)\end{array}$ & a) Gas name: & a) Yes & $\begin{array}{l}\text { a) Yes } \\
\text { b) No (indicate your } \\
\text { reason in 'comment' } \\
\text { section below) } \\
\text { Comment: }\end{array}$ & $\begin{array}{l}\text { a) Yes } \\
\text { b) No (list in the } \\
\text { 'comment' section below } \\
\text { the gases with which it's } \\
\text { stored) } \\
\text { Comment: } \\
\text { c)N/A }\end{array}$ & $\begin{array}{l}\text { a) Yes } \\
\text { b) No (than comment } \\
\text { on how it's stored) } \\
\text { Comment (how is it } \\
\text { stored if not in one of } \\
\text { the above manner?): }\end{array}$ \\
\hline
\end{tabular}


Appendix C: Safety Inspection Checklists Form \#1

\section{PARTA: Obtain answers to the following Yes No Comment questions from lab supervisor Question}

1. A. Does your lab have a biosafety certificate?

Note: only ask this if you're inspecting a biology

lab

1. B. If "yes", what is the Containment/Biosafety Level (C/BL) of the lab? $\square \mathrm{C} / \mathrm{BL} 1$

2. Is there a lab safety manual available that the lab follows?

2. B. How can lab personnel have access to the lab safety manual?

\section{Answer:}

3. A. Are Material Safety Data Sheets (MSDSs) available? If so, than indicate in the 'comment' section, how or where lab workers can have access to them

3. B. If MSDSs are available, check their date and indicate in the comment section, whether the sheets are up-to-date, i.e. less than 3 years old.

4. Are there supplies provided to allow for decontamination or cleaning before and after an experiment (i.e. Bleach /Alcohol)?

\section{Part B: To be conducted inside the laboratory}

\section{Housekeeping and general safety \\ Y N N/A Comments}

1. The lab doors are kept closed

2. Aisles (passage ways) are clean

1. Aisles (passage ways) are free of obstructions

2. Floors are clean, e.g. no spill on them

3. Floors are unobstructed

4. Shelves are uncluttered

5. Shelves are free of signs of damage (e.g., sagging, cracking, etc.) 
6. Lab benches are clean, Y i.e. no spill on them

\section{N N/A Comments}

7. Lab benches are clear of unnecessary items, e.g. worker's bag or clothes

8. Hand-washing sinks are available

9. Hand-washing sinks are clean

10. Hand-washing sinks are unobstructed

11. Overall, is the general appearance of the lab clean?

12. Overall, is the general appearance of the lab orderly (i.e. neat and tidy)?

\section{Personal Protective Equipment (PPE)}

1. Lab coats are available

2. Lab coats are hanged in $\mathbf{Y}$ an area separate from street clothes

3. Gloves are available

4. Eye protection (goggles or safety glasses) are available 
5. All the above PPE are in good condition, e.g. not ripped or broken, not stained or tainted, etc.

\section{Safety and Emergency Equipment}

1. Safety showers are available

2. Safety showers are clean

3. Safety showers are unobstructed

4. Safety Showers are tested regularly (check the tag on them for a testing date)

5. Safety Showers are regularly inspected (check the tag on them for the inspection date)

6. Eyewash stations are available

\section{Y N N/A Comments}

7. Eyewash stations are unobstructed

8. Eyewash stations are clean

9. Eyewash stations are tested periodically (check the tag on them for a testing date)

10. Eyewash stations are regularly inspected (check the tag on them for the inspection date)

11. Chemical fume hood(s) is/are available

12. Chemical fume hood(s) 
is/are clean

13. Chemical fume hood(s) is/are uncluttered, e.g. no storage of items unless in use

14. There is a label on the fume hoods indicating that the hood(s) has/have been tested within the last year

15. Biological Safety

\section{Y N N/A Comments}

Cabinet(s) (BSC) is/are available

16. Biological Safety Cabinet(s) (BSC) is/are clean

17. Biological Safety Cabinet(s) (BSC) is/are uncluttered

18. There is a label on the Biosafety Cabinet(s) indicating that it/they has/have been tested within the last year

19. Stocked first aid kit is available

20. Stocked spill cleanup kit is available

21. Fire alarm pull stations are available

22. Fire alarm pull stations are unobstructed

23. Fire extinguishers are present

24. Fire extinguishers are 
mounted on a wall

25. Fire extinguishers are stored in a cabinet

26. Fire extinguishers are unobstructed

27. Exit doors are unobstructed

28. Entrance doors are unobstructed

Hazardous substances labeling and storage (per WHMIS legislated requirements)

1. All chemical containers are labeled either with a supplier or workplace label

2. All biological containers are labeled indicating container content

3. All storage areas (e.g. shelves, refrigerators, cabinets, etc.) are clearly labeled

4. All storage areas are clean

5. All storage areas are uncluttered

6. Each storage area is categorized/grouped based on hazard class, e.g. acids cabinets, flammable cabinets

7. No chemicals are stored Y N N/A Comments closed to heat sources e.g. direct sunlight via window etc.

8. No biological agents are stored closed to heat 
sources e.g. direct

sunlight via window etc.

Signage, emergency safety information, and

lab security

1. Emergency contact

information (name and

phone number) are

posted in the laboratory

2. After-hours emergency contact information for lab staff are posted in the laboratory

3. Emergency contact information (name and phone number) are up-todate

4. After-hours emergency contact information for lab staff are up-to-date

5. Exits are clearly marked

6. Safety Shower(s) are identified by a highly visible sign

7. Eyewash stations are identified by a highly visible sign 
Appendix D: Safety Inspection Checklist form \#2

Part A: All Answers for these three questions to be obtained from lab supervisor

\section{Questions}

1. Does the lab have a manual for proper waste handling, storage, and disposal?

2. Are lab workers aware of this manual?

3. For those new in the lab, where can they access the manual?

\section{Answers}

a) Yes

b) No

a) Yes

b) No

\section{Comments}

4. Are there waste (hazardous

a) Yes and non-hazardous) containers available in the lab, e.g. waste bins, bottles, or pails?

5. If the lab has waste pails, are they color-coded?

b) No (then check N/A for the remaining questions)

a) Yes (than indicate the color(s) in the 'comment section)

b) No

c) N/A

6. Are the other waste

a) Yes container(s) labeled, indicating

b) No their content?

c) N/A

7. What is inside the waste container(s), as per label? Please check all that apply a)

Refuse/garbage

b) Papers

c) Plastics

c) Hazardous

waste

e)

Biohazardous

waste

f) Sharps

g) Broken glass

h) Other

(please specify) 
8. Is there a lid on the waste container(s)?

a) Yes

b) $\mathrm{No}$

c) N/A

9. Where in the lab is/are the waste container(s) stored?

a) On the floor away from egress routes b) On the floor and blocking egress routes c) On benchtop d) In fume hoods e) In biosafety cabinet f) Other (please specify)

e) N/A

10. Is/are the waste container(s) in good condition, e.g. not corroded, broken, leaking, etc.? a) Yes

b) No (then comment on its condition as you see it)

c) N/A

a) Yes

b) $\mathrm{No}$

c) N/A

a) $\mathrm{Yes}$

b) $\mathrm{No}$

c) N/A

a) $\mathrm{Yes}$

b) No

c) N/A

a) $\mathrm{Yes}$

b) $\mathrm{No}$

c) $\mathrm{N} / \mathrm{A}$

contaminated papers,

food waste, etc., stored in a separate area from any hazardous and toxic waste materials? 
Appendix E: Excerpt from University X's Workplace Inspection Program

\section{Workplace Inspection - Preliminary Information}

\section{Building Name:}

List Schools or Department using the area(s):

List Individual Responsible for the area(s):

List Departmental Safety Officer for the area(s):

Telephone Ext:

Time and date of Inspection:

Confirmation letter to Responsible individual advising of date of Workplace Inspection: (date sent) Copy of previous Workplace Inspection: (month, year)

Workplace Inspection team:

Worker Representative

Qualified Individual

DSO representing (Department, School)

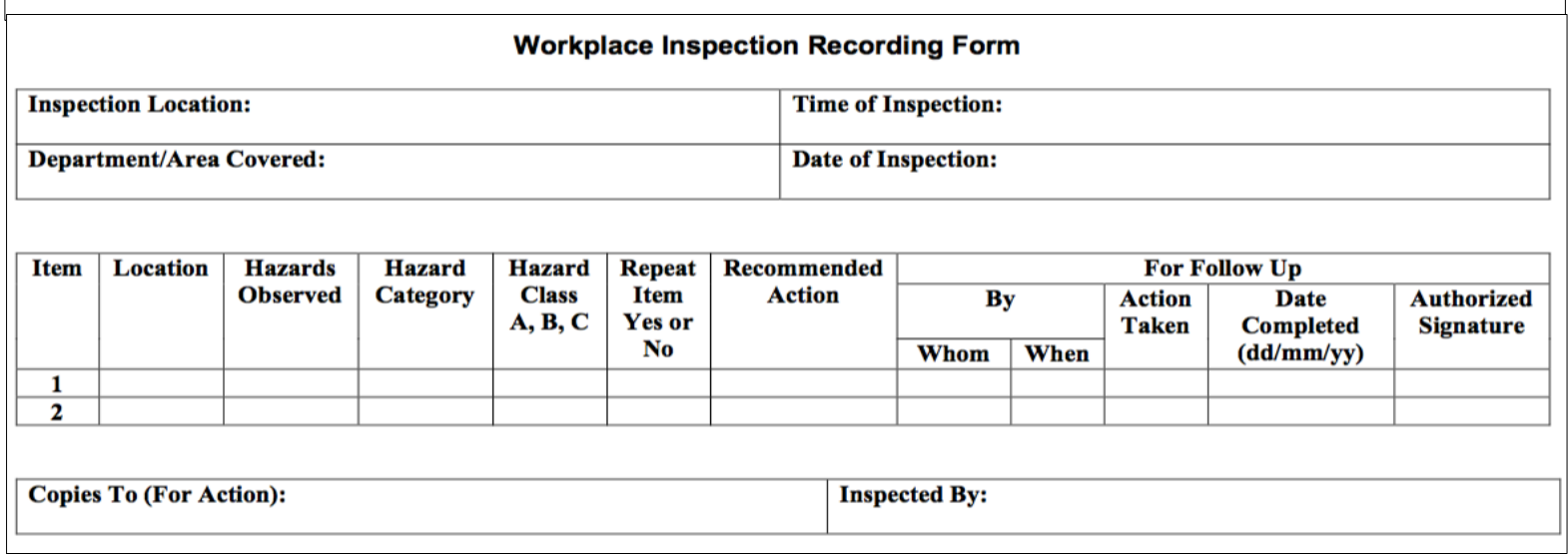


Appendix F: Survey

Part A: Introduction

A1. What is your age?

a.Under 18 years

b. 18-20 years

c. $21-25$ years

d.26-30 years

e. 31-35 years

f.36-40 years

g. $41-50$ years

h. $51-60$ years

i.More than 60 years

A2. What is your sex?

a.Female

b.Male

A3. What is your current position at University $\mathbf{X}$ ?

a.Research assistant

b.Graduate student

c.Lab supervisor/manager

d.Technician

e.Faculty

f.Other (please specify)

A4. What department are you currently working in?

a.Chemistry

b.Biology

c.Engineering

d.Other (please specify)

A5. Including yourself, how many people work in your lab?

a.1-5 staff

b.6-10 staff

c. 11-20 staff

d.21-100 staff

A6. How long have you been working in your current lab?

a. Less than 5 months

b.5 -11 months

c. 1-2 years

d. 3-4 years

e.5-10 years

f. $11+$ years

A7. How long have you worked in a lab setting (including your current lab and any prior experience)?

a. Less than 5months

b.5 -11 months

c. 1-2 years

d.3-4 years

e.5-10 years

f. $11+$ years

A8. What type of lab do you currently work in? 
a.Wet lab- where chemicals, drugs, or other biological matter are tested and analyzed using liquids i.e. use of chemical solutions

b.Dry lab- where computers or computer generated models are used for analysis. E.g. using a downloadable biological software for biological analysis

c.Both wet and dry lab

A9. If you work with chemical substances, how many hours per week do you spend working with these substances?

a.1-10hrs/week

b. 11-20hrs/week

c. 21-30hrs/week

d.31-40hrs/week

e.40hrs/week or more

f. Not applicable

A10. If you work with biological substances, how many hours per week do you spend working with these substances?

a.1-10hrs/week

b.11-20hrs/week

c. 21-30hrs/week

d.31-40hrs/week

e. $40 \mathrm{hrs} /$ week or more

f. Not applicable

Part B: Attitude towards safety

B1. I feel that my lab is a safe place to work

a.Strongly Agree

b.Agree

c.Neither agree nor disagree

d.Disagree

e.Strongly disagree

f .I don't know

B2. Personally speaking, how important is lab safety to you

a.Very important

b.Quite important

c.Not very important

d.Not important at all

e.I don't know

B3. Which of the following statements best describes your laboratory in regards to safety?

a.Safety is paramount and takes precedence over all other lab priorities

b.Safety is very important

c.Safety is of equal importance to other lab priorities

d.Safety is less important than other lab priorities

e.Safety is a low priority in my lab

f. I don't know

B4. I believe that the level of risk of the work conducted in my lab is

a.Very high risk

b.High risk

c.Moderate risk

d.Low risk

e. Very low risk

f.I don't know

B5. In your opinion, which of the following parties should be concerned about lab safety? Check all that apply 
a.Lab supervisor/ manager
b.Faculty
c.Technicians
d.Students
e.I don't know
f.Other: please specify

B6. How do you feel about the safety procedures in your current lab?

a.Far too stringent

b.A little too stringent

c.About right

d. Should be a little more stringent

e.Should be far more stringent

f.I don't know

B7. Lab safety rules and regulations negatively impact my lab productivity

a.Strongly Agree

b.Agree

c.Neither agree nor disagree

d.Strongly disagree

e.Disagree

f.Neither agree nor disagree

B8. To what extent, if any, do you think that lab inspections affect the safety of a lab?

a.Safety is greatly improved by the inspections

b.Safety is slightly improved by the inspections

c. The inspections have no significant impact on safety

d.Safety is slightly compromised by the inspections

e.Safety is greatly compromised by the inspections

f.I don't know

B9. I think that the overall safety in my lab could be improved

a.Strongly agree

b.Agree

c.Neither agree nor disagree

d.Disagree

e.Strongly disagree

$\underline{\text { Part C: Safety in your lab }}$

C1. My lab always has sufficient supplies of personal protective equipment (PPE) that I need for my lab work a.Strongly Agree

b.Agree

c.Neither agree nor disagree

d.Disagree

e.Strongly disagree

f.I don't know

C2. When performing lab work, how often do you wear personal protective equipment (PPE)?

a.All the time

b.Most of the time

c.Some of the time

d.Rarely

e.Never

f.I don't know

C3. In general, I feel that I am able to use the required personal protective equipment (PPE) properly a.Strongly Agree

b.Agree 
c.Neither agree nor disagree

d.Disagree

e.Strongly disagree

f.I don't know

C4. How would you rate the level of access to safety equipment and materials such as personal protective equipment (PPE), Material Safety Data Sheets (MSDS), eye wash stations, etc. in your lab?

a.Easily accessible

b.Somewhat accessible

c.Neither accessible nor inaccessible

d.Somewhat inaccessible

e.Not accessible

I don't know

C5. The safety equipment that my lab uses (chemical fume hoods, biosafety cabinets, etc) are always certified and in good working order

a.Strongly Agree

b.Agree

c.Neither agree nor disagree

d.Disagree

e.Strongly disagree

f.I don't know

C6. The research equipment I use for my lab work is safe

a.Strongly agree

b.Agree

c.Neither agree nor disagree

d.Disagree

e.Strongly disagree

f.I don't know

C7. I am aware of and understand the minimum training requirements for my specific lab duties

a.Strongly agree

b.Agree

c.Neither agree nor disagree

d.Disagree

e.Strongly disagree

f.I don't know

C8. Who has provided you with lab safety training in your current lab? Check all that apply

a.Lab safety staff from University $\mathrm{X}$ 's health and safety department(s)

b.A co-worker

c. Supervisor or principal investigator

d.Other (please specify)

e.I have not received any training.

If you answered " $e$ ", please skip to question C13

C9. I received safety training on the specific agent/hazards I work with

a.Strongly Agree

b.Agree

c.Neither agree nor disagree

d.Disagree

e.Strongly disagree

f.I don't know 
$\mathrm{C} 10$. I received sufficient safety training in order to effectively minimize the risk of injury to myself and others in the lab

a.Strongly Agree

b.Agree

c.Neither agree nor disagree

d.Disagree

e.Strongly disagree

f.I don't know

$\mathrm{C} 11$. I received sufficient safety training in order to be compliant with the rules and regulations related to my lab duties

a.Strongly Agree

b.Agree

c.Neither agree nor disagree

d.Disagree

e.Strongly disagree

f.I don't know

C12. Which, if any, of the following are used to communicate lab safety -either within your lab or by University $\mathrm{X}$ ? Check all that apply.

a.Direct lab training through the principal investigator/supervisor

b.Online resources (websites, online training, quizzes etc)

c.Classroom based training

d.Reading standard operating procedures

e.Visuals such as flyers and posters on lab safety

f.Lab safety videos

g.Lab safety is not communicated in my lab, at least to my knowledge

h.Other: please specify

i.I don't know

C13. I feel comfortable speaking to my supervisor or principal investigator about safety concerns regarding my own lab work

a.Strongly agree

b.Agree

c.Neither agree nor disagree

d.Disagree

e.Strongly disagree

f.I don't know

$\mathrm{C} 14$. In the time that you've been at your current lab, have you ever sustained an injury of any kind?

a.No. If no, please skip to question C18

b.Yes, once

c.Yes, on more than one occasion

d.I don't know

C15. What was the nature of your injury or injuries? Check all that apply

a.Minor laceration/cut/bite requiring no stitches

b.Laceration/cute/bite requiring stitches

c.Needle stick

d. Thermal burn

e.Chemical burn

f.Chemical inhalation

g.Other: please specify

h.I don't remember

C16. I have sustained an injury myself that I have not reported to my supervisor or principal investigator 
a.This has happened only once

b.This has happened on more than one occasions

c.This has never happened

d.I don't know

C17. In your current lab, are you aware of what to do in case of emergencies such as fires, spills etc.?

a.Yes

b.No

c.I don't know

C18. Which, if any, type of risk assessment do you currently carry out before conducting an experiment?

a.I conduct a risk assessment using my lab's approved form

b.I conduct a risk assessment using a formalized, third-party hazard/risk assessment tool

c.I conduct a risk assessment using my own format

d.I informally assess risk

e.No risk assessment is performed

C19. On average, how frequently are there people conducting experiments in your current lab while working alone?

a.Every day

b.At least once a week

c.A couple of times a month

d.Less than once a month

e.I don't know

C20. My supervisor/lab manager/PI regularly checks to make sure I am performing my laboratory duties in a safe fashion using proper safety equipment

a.Strongly agree

b.Agree

c.Neither agree nor disagree

d.Disagree

e.Strongly disagree

Part D: Additional questions for those with supervisory responsibilities (e.g. managers, faculty etc.)

D1.What are the three most frequently handled chemical agents, if any, in your lab?

D2.What are the three most frequently handled biological agents, if any, in your lab

D3. Members of my lab are aware of and understand the minimum training requirements for their specific lab duties

a.Strongly agree

b.Agree

c.Neither agree nor disagree

d.Disagree

e.Strongly disagree

f.I don't know

D4. Appropriate safety measures in my lab have been taken to protect employees from injury a.Strongly agree

b.Agree

c.Strongly disagree

d.Neither agree nor disagree

e.Disagree

f.I don't know 
D5. Who in your lab is primarily responsible for ensuring compliance with lab safety requirements?

a.The lab manager or a senior technician

b. The principal investigator or head supervisor

c.Departments' safety officer

d.The lab safety staff from University X's health and safety department

e.A graduate student

f.A junior technician

g.No one has been assigned or specifically assumed this responsibility

h.I don't know

D6. I have access to the data and records which are tracked regarding my lab's safety and compliance

a.Strongly Agree

b.Agree

c.Neither agree nor disagree

d.Disagree

e.Strongly disagree

D7. How frequently are safety inspections carried out for your lab?

a.At least once a month

b.At least once a quarter

c.At least once per year

d.At least once every two years

e.Less than once every two years

f.They are carried out but I don't know how often

g.To the best of my knowledge, lab safety inspections are not carried out

h.I don't know

D8. How many major injuries/minor injuries are you aware of that have occurred in your current lab in the past 12 months?

a.Number of major injuries: ones that would require attention from a medical professional e.g. nurse, doctor, paramedic

i.None

ii. 1

iii.2

iv. 3

v. 4

vi.5+

b.Number of minor injuries: ones that could be dealt with by a first aider, colleague or yourself.

i.None

ii. $1-2$

iii. $3-5$

iv. $6-10$

v. $10+$

D9. The number of major injuries could have been reduced if lab safety procedures were always followed a.Strongly agree

b.Agree

c.Neither agree nor disagree

d.Disagree

e.Strongly disagree

f.I don't know

g.Not applicable

D10. The number of $\underline{\text { minor }}$ injuries could have been reduced if lab safety procedures were always followed a.Strongly agree

b.Agree

c.Neither agree nor disagree 
d.Disagree

e.Strongly disagree

f.I don't know

g.Not applicable

D11. If I have concerns, I feel comfortable speaking to students or lab staff about their lab safety practices a.Strongly agree

b.Agree

c.Neither agree nor disagree

d.Disagree

e.Strongly disagree

f.Not applicable

D12. What are the three most significant barriers to improving safety in your laboratory? Check three only a.Time and hassle factors

b.Apathy

c.Lack of understanding of safety requirements

d.Lack of leadership

e.Focus on compliance requirements over safety

f.Competing priorities

g.Lack/inadequate safety training

h.Lack of funds for safety equipment

i.Untrained staff

j.Inadequate facilities

k. There are no significant barriers

1.Inadequate equipment

m.Other (specify) 


\section{References}

Abbas, M., Zakaria, A. M., \& Balkhyour, M. A. (2015). Investigation of safety facilities and safe practices in chemical laboratories of a Saudi university. Advpub. Https://doi.org/10.11162/daikankyo.E15RP1001

Adane, L., \& Abeje, A. (2012). Assessment of Familiarity and Understanding of Chemical Hazard Warning Signs among University Students Majoring Chemistry and Biology: A Case Study at Jimma University, Southwestern Ethiopia. IDOSI Publications, 10 (290-299).

Allen, R. O. (1983). Waste disposal in the laboratory: Teaching responsibility and safety. J. Chem. Educ, 60(3), A81.

Allen, k. (2014). A young lab worker, a professor and a deadly accident | Toronto Star. Retrieved from https:/www.thestar.com/news/world/2014/03/30/a_young_lab_worker_a_professor_and_a_deadly_accident.html American Chemical Society. (2003). Safety in academic chemistry laboratories (7th ed.). Washington, DC.

Retrieved from https://www.acs.org/content/dam/acsorg/about/governance/committees/chemicalsafety/publications/safetyin-academic-chemistry-laboratories-faculty.pdf

American Chemical Society. (2012). Creating Safety Cultures in Academic Institutions: a Report of the Safety Culture Task Force ACS Committee on Chemical Safety (First edition). Retrieved from https:/www.acs.org/content/dam/acsorg/about/governance/committees/chemicalsafety/academic-safetyculture-report-final-v2.pdf

Anderson, R. G. (2013). Chemistry laboratories, and how they might be studied. Studies in History and Philosophy of Science Part A, 44(4), 669-675.

Armour, M. A. (2016). Hazardous laboratory chemicals disposal guide. CRC press

Artdej, R. (2012). Investigating Undergraduate Students' Scientific Understanding of Laboratory Safety. Procedia Social and Behavioral Sciences, 46, 5058-5062. Https://doi.org/10.1016/j.sbspro.2012.06.385

Asimov, I., Burke, J., \& Bergman, J. (2013). The Impact of Science on Society. (W. Seager \& K. Toppert, Eds.). Penny Hill Press Inc,.

Backus, B. D., Fivizzani, K., Goodwin, T., Finster, D., Austin, E., Doub, W., ... Kinsley, S. (2012). Laboratory safety culture: Summary of the chemical education research and practice-Safety in chemistry education 
panel discussion at the 46th Midwest and 39th Great Lakes Joint Regional American Chemical Society Meeting, St. Louis, Missouri, on October 21, 2011. Journal of Chemical Health and Safety, 19(4), 20-24.

Bagley, M. (2013, August 14). Marie Curie: Facts \& Biography. Retrieved August 17, 2016, from http://www.livescience.com/38907-marie-curie-facts-biography.html

Balas, F., Arruebo, M., Urrutia, J., Santamaria, J., \& others. (2010). Reported nanosafety practices in research laboratories worldwide. Nature Nanotechnology, 5(2), 93.

Bell, J. T., \& Fogler, H. S. (2001). Virtual reality laboratory accidents. In Proceedings of American Society for Engineering Education Annual Conference, Albuquerque, NM, American Society for Engineering Education. Retrieved from https://www.cs.uic.edu/ jbell/Professional/Papers/ASEE2001.pdf

Benderly, B. L. (2009, May 22). What makes academic laboratories such dangerous places to work? Retrieved July 29,2016 , from http://www.slate.com/articles/health_and_science/science/2009/05/explosions_in_the_lab.html

Benderly, B. L. (2014, July 9). The Landmark Patrick Harran Case Ends Inconclusively. Retrieved November 17, 2016, from http://www.sciencemag.org/careers/2014/07/landmark-patrick-harran-case-ends-inconclusively

Benderly, B. L. (2015, April 30). Death in the Lab | discovermagazine.com. Discover Magazine. Retrieved from http:/discovermagazine.com/2015/june/20-death-in-the-lab

Benderly, B. L. (2013, June 5). Study to Examine Academic Lab-Safety Culture. Science | AAAS. Retrieved from http://www.sciencemag.org/careers/2013/06/study-examine-academic-lab-safety-culture

Benderly, B. L. (2016a, March 24). Lab explosion maims University of Hawaii postdoc. Retrieved November 8 , 2016, from http://www.sciencemag.org/careers/2016/03/lab-explosion-maims-university-hawaii-postdoc

Benderly, B. L. (2016b, July 6). Report on UH lab explosion reveals deep, systemic safety failures. Retrieved November 8, 2016, from http://www.sciencemag.org/careers/2016/07/report-uh-lab-explosion-revealsdeep-systemic-safety-failures

Benderly, B. L. (2010, August 1). Danger in School Labs: Accidents Haunt Experimental Science. Scientific American. Retrieved from http://www.scientificamerican.com/article/danger-in-school-labs/

Berkeley. (2013). Drain Disposal Restrictions for Chemicals (p. 21). University of California. Retrieved from http://ehs.berkeley.edu/sites/default/files/lines-of-services/hazardous-materials/draindisposal.pdf

Berkeley Lab. (2000, January 14). Fume Hood: Advanced, Energy-Efficient Fume Hood Under Development. 
Retrieved July 28, 2016, from http://www2.lbl.gov/Science-Articles/Archive/fume-hood-advances.html Brigham Young University. (2016). Life Sciences Safety-Safety Policies. Retrieved December 6, 2016, from http://lifesciences.byu.edu/safety/labsafety/safetypolicies

Blackman, W. (2001). Basic Hazardous Waste Management, Second Edition (2nd ed.). CRC Press. Retrieved from http://www.crenetbase.com/doi/book/10.1201/9781420032604

Blaszczak-Boxe, A. (2016, July 6). Hawaii lab explosion caused by static discharge | Chemistry World. Retrieved July 16, 2016, from http://www.rsc.org/chemistryworld/2016/07/hawaii-lab-explosion-static-electricitysafety-failings

Bliss, M. (2015, August 19). The Discovery of Insulin. In The Canadian Encyclopedia. Retrieved from http://www.thecanadianencyclopedia.ca/en/article/the-discovery-of-insulin/

Borchardt, J. K. (2006, August 4). Lab Safety Requires Training and Commitment | Science | AAAS. Science | AAAS. Retrieved from https://www.sciencemag.org/careers/2006/08/lab-safety-requires-training-andcommitment

Burnett, L. C., Lunn, G., \& Coico, R. (2009). Biosafety: Guidelines for Working with Pathogenic and Infectious Microorganisms. In R. Coico, A. Mcbride, J. M. Quarles, B. Stevenson, \& R. K. Taylor (Eds.), Current Protocols in Microbiology (p. 1A.1.1-1A.1.14). Hoboken, NJ, USA: John Wiley \& Sons, Inc. Retrieved from http://doi.wiley.com/10.1002/9780471729259.mc01a01s13

Canadian Association of University Teachers. (2011, November). Academic Freedom. Retrieved January 12, 2017, from https://www.caut.ca/about-us/caut-policy/lists/caut-policy-statements/policy-statement-on-academicfreedom

Canadian Centre for Occupational Health and Safety. (2009, March 1). Effective Workplace Inspections: OSH Answers. Retrieved November 22, 2016, from https:/www.ccohs.ca/oshanswers/prevention/effectiv.html Canadian Centre for Occupational Health and Safety. (2009a, February 2). Risk Assessment: OSH Answers. Retrieved November 11, 2016, from https://www.ccohs.ca/oshanswers/hsprograms/risk_assessment.html

Canadian Centre for Occupational Health and Safety. (2009b, November 20). Hazard and Risk: OSH Answers. Retrieved November 11, 2016, from http://www.ccohs.ca/oshanswers/hsprograms/hazard_risk.html Canadian Centre for Occupational Health and Safety. (2010, March 7). WHMIS 2015 - Hazard Classes and Categories: OSH Answers. Retrieved November 11, 2016, from 
https://www.ccohs.ca/oshanswers/chemicals/whmis_ghs/hazard_classes.html

Canadian Centre for Occupational Health and Safety. (2012, January 18). WHMIS 1988 - General: OSH Answers.

Retrieved February 19, 2017, from http://www.ccohs.ca/oshanswers/legisl/intro_whmis.html

Canadian Centre for Occupational Health and Safety. (2016a, March 7). WHMIS 2015 - Education and Training:

OSH Answers. Retrieved January 16, 2017, from

https://www.ccohs.ca/oshanswers/chemicals/whmis_ghs/education_training.html

Canadian Centre for Occupational Health and Safety. (2016b, March 7). WHMIS 2015 - Labels : OSH Answers.

Retrieved November 11, 2016, from https://www.ccohs.ca/oshanswers/chemicals/whmis_ghs/labels.html

Canadian Centre for Occupational Health and Safety. (2017a, February 2). Hazard and Risk: OSH Answers.

Retrieved November 11, 2016, from http://www.ccohs.ca/oshanswers/hsprograms/hazard_risk.html

Canadian Centre for Occupational Health and Safety. (2017b, February 22). Emergency Showers and Eyewash

Stations: OSH Answers. Retrieved February 23, 2017, from

https://www.ccohs.ca/oshanswers/safety_haz/emer_showers.html

Canadian Centre for Occupational Health and Safety. (2016a, March 7). WHMIS 2015 - Labels: OSH Answers.

Retrieved November 11, 2016, from https://www.ccohs.ca/oshanswers/chemicals/whmis_ghs/labels.html

Canadian Centre for Occupational Health and Safety. (2016b, March 7). WHMIS 2015 - Safety Data Sheet (SDS):

OSH Answers. Retrieved November 11, 2016, from

https://www.ccohs.ca/oshanswers/chemicals/whmis_ghs/sds.html

Canadian Centre for Occupational Health and Safety. (2016, March 7). WHMIS 2015 - Pictograms: OSH Answers.

Retrieved November 11, 2016, from

https://www.ccohs.ca/oshanswers/chemicals/whmis_ghs/pictograms.html

Carrillo, R. A. (2002). Safety leadership formula: Trust + credibility x competence = results. Professional Safety; Des Plaines, 47(3), 41-47.

Chemical Heritage Foundation. (2016, January 15). Louis Pasteur. Retrieved from https://www.chemheritage.org/historical-profile/louis-pasteur

Chen, N., Ishida, Y., Mishina, T., Matsunami, A., \& Murata, S. (2016). Fume hood management in Nagoya

University. 7(2), 125-128. Https://doi.org/10.11162/daikankyo.E15PROCP16

Chien, Y.-C., Chang, C.-P., \& Lo, C.-P. (2009). Assessment of Air Change Rates in Chemical Laboratories. Indoor 
and Built Environment, 18(2), 162-167.

Chosewood, L. C., \& Wilson, D. E. (2009). Biosafety in Microbiological and Biomedical Laboratories. U.S. Department of Health and Human Services, Public Health Service, Centers for Disease Control and Prevention, National Institutes of Health.

Choudhry, R. M., Fang, D., \& Mohamed, S. (2007). The nature of safety culture: A survey of the state-of-the-art. Safety Science, 45(10), 993-1012. Http://doi.org/10.1016/j.ssci.2006.09.003

Christensen, K. (2009, March 1). Deadly UCLA lab fire leaves haunting questions. Los Angeles Times. Retrieved from http://articles.latimes.com/2009/mar/01/local/me-uclaburn1

Ciloski, B. (2005). A Guide to Fume Hood Codes and Standards. Retrieved February 23, 2017, from http://www.laboratorydesign.com/public/guild_fume.html

Concordia University. (2017). Laboratory safety manual. Retrieved October 18, 2016, from https:/www.concordia.ca/content/dam/concordia/services/safety/docs/EHS-DOC001_laboratorysafetymanual.pdf

Cooper Ph.D., M. D. (2000). Towards a model of safety culture. Safety Science, 36(2), 111-136. Http://doi.org/10.1016/S0925-7535(00)00035-7

Cotter, P., \& Witherspoon, R. (2016). Texas Tech University: Office of the Vice President for Research. Retrieved November 1, 2016, from http://www.depts.ttu.edu/vpr/faculty/scholarlymessenger/stories/2013/Feb/labaccidents.php

Côté, R., Davis, H., Dimock, C., Korpan, M., Loewen, K., \& Segal, L. M. (1998). The evaluation and hazard classification of toxicological information for workplace hazardous materials information system material safety data sheets. Regulatory Toxicology and Pharmacology, 27(1), 61-74.

Creighton University. (n.d.). Storing chemicals in fume hoods | Environmental Health \& Safety. Retrieved October 19, 2016, from http:/www.creighton.edu/researchservices/ehs/labsafety/storingchemicalsinfumehoods/

Cyranoski, D. (2015). Postdoc dies in lab fire at Tsinghua University. Nature. Https://doi.org/10.1038/nature.2015.19066

Dartmouth College. (2004). Chemlab - Safety - Safety Hazards in the Lab. Retrieved November 2, 2016, from https://www.dartmouth.edu/ chemlab/info/safety/hazards.html

Dong, J. (2016, September 21). Three postgraduates of Donghua University injured in campus lab blast. Retrieved 
from http:/www.shanghaidaily.com/metro/society/Two-students-severely-injured-in-lab-blast-in-localDonghua-University/shdaily.shtml

Eldridge, R. (2012). An Analysis of Laboratory Inspections at the University of Kentucky. MPA/MPP Capstone Projects. Retrieved from http://uknowledge.uky.edu/mpampp_etds/63

Engida, T. (2011). Chemical Safety in Laboratories of African Universities. African Journal of Chemical Education, $1(2), 35-49$.

Euromed Info. (n.d.). The Health Belief Model. Retrieved December 5, 2016, from http://www.euromedinfo.eu/thehealth-belief-model.html/

Experiment. (n.d.). Retrieved from http://www.dictionary.com/browse/experiment

Environmental Health \& Safety. (2014). Laboratory Accidents | Environmental Health \& Safety. Retrieved November 1, 2016, from http://www.ehs.ucsb.edu/labsafety/laboratory-accidents

Ferjencik, M., \& Jalovy, Z. (2010). What can be learned from incidents in chemistry labs. Journal of Loss Prevention in the Process Industries, 23(5), 630-636. Https://doi.org/10.1016/j.jlp.2010.06.009

Finucane, M. L., Slovic, P., Mertz, C. K., Flynn, J., \& Satterfield, T. A. (2000). Gender, race, and perceived risk: The'white male'effect. Health, Risk \& Society, 2(2), 159-172.

Flynn, J., Slovic, P., \& Mertz, C. K. (1994). Gender, Race, and Perception of Environmental Health Risks. Risk Analysis, 14(6), 1101-1108. Https://doi.org/10.1111/j.1539-6924.1994.tb00082.x

Foster, B. L. (2003). Principles of laboratory safety management in academia. Chemical Health and Safety, 10(2), 13-16. Https://doi.org/10.1016/S1074-9098(02)00457-4

Foster, B. L. (2004). Laboratory safety program assessment in academia. Chemical Health and Safety, 11(5), 6-13. Https://doi.org/10.1016/j.chs.2004.05.016

Foster, B. L. (2005). The Chemical Inventory Management System in academia. Chemical Health and Safety, 12(5), 21-25. Http://doi.org/10.1016/j.chs.2005.01.019

Fred Hutchinson Cancer Research Center. (n.d.). 8.0 Chemical Fume Hoods. Retrieved August 17, 2016, from https://extranet.fhcrc.org/EN/sections/ehs/hamm/chap3/section8.html

Frieden, T., Jaffe, H., Stephens, J., Thacker, S., \& Zaza, S. A. (2011). Fatal laboratory-acquired infection with an attenuated Yersinia pestis Strain--Chicago, Illinois, 2009. MMWR. Morbidity and Mortality Weekly Report, 60(7), 201-205. 
Lab Crafters. (n.d.). Fume Hoods. Retrieved November 21, 2016, from http://www.labcrafters.com/fumehoods/whatisfume.html

Furr, A. K. (2000). CRC Handbook of Laboratory Safety 5th Edition (5th ed.). CRC Press LLC.

Gadd, S., \& Collins, A. M. (2002). Safety culture: A review of the literature. Health and Safety Laboratory.

Gallion, L. A., Samide, M. J., \& Wilson, A. M. (2015). Demonstrating the importance of cleanliness and safety in an undergraduate teaching laboratory. Journal of Chemical Health and Safety, 22(5), 28-31. Https://doi.org/10.1016/j.jchas.2015.01.002

Genthon, P., Leigh, T., Bremer, C., \& Pearce, T. (2010, November 25). French chemistry professor fined for deadly lab blast. Reuters. Retrieved from http://www.reuters.com/article/us-france-professoridustre6ao2n720101125

Gibbs, L. M. (2005). Chemtracker Consortium - The higher education collaboration for chemical inventory management and regulatory reporting, 9-14. Http://doi.org/10.1016/j.chs.2005.01.017

Gibson, J. H., Schröder, I., \& Wayne, N. L. (2014). A research university’s rapid response to a fatal chemistry accident: Safety changes and outcomes. Journal of Chemical Health and Safety, 21(4), 18-26. Https://doi.org/10.1016/j.jchas.2014.01.003

Government of Canada, P. H. A. Of C. (2015, March 11). Canadian Biosafety Standard (CBS), Second Edition Canadian Biosafety Standards and Guidelines. Retrieved July 12, 2016, from http://canadianbiosafetystandards.collaboration.gc.ca/cbs-ncb/index-eng.php

Government of Canada, P. H. A. Of C. (2016). Canadian Biosafety Standards and Guidelines. Retrieved July 12, 2016, from http://canadianbiosafetystandards.collaboration.gc.ca/

Government of Canada, P. H. A. Of C. (2004). Chapter 2: Biological Safety - Laboratory Biosafety Guidelines: 3rd Edition 2004 (3rd ed.). Retrieved from http://www.phac-aspc.gc.ca/publicat/lbg-ldmb1-04/ch2eng.php\#jmp-lan22

Government of Ontario. (2016). Occupational Health and Safety Act, R.S.O. 1990, c. O.1, § 54. Retrieved from https://www.ontario.ca/laws/view

Government of Ontario, M. Of L. (2016). Explosion Risk Associated with Old Laboratory Samples | Ministry of Labour. Retrieved from https://www.labour.gov.on.ca/english/hs/pubs/alerts/a35.php

Government of Ontario. R.R.O. 1990, Reg. 860: Workplace hazardous materials information system (WHMIS) 
(2014). Retrieved from https://www.ontario.ca/laws/view

Government of ontario, m. Of 1. (n.d.). Chapter 4. WHMIS and the employer | workplace hazardous Materials

Information System: A Guide to the Legislation | Ministry of Labour. Retrieved from

https:/www.labour.gov.on.ca/english/hs/pubs/whmis/whmis_4c.php

Government of Ontario. Law Document English View, § 4 (2014). Retrieved from https://www.ontario.ca/laws/view

Government of Northwest Territories. (2011). A Guide to the spill contingency planning and reporting regulations.

PDF retrieved from

http://www.enr.gov.nt.ca/sites/default/files/reports/guide_to_spill_contingency_planning_and_reporting.pd f

Grady, D. (2014, July 19). Pathogen Mishaps Rise as Regulators Stay Clear. The New York Times. Retrieved from http:/www.nytimes.com/2014/07/20/science/pathogen-mishaps-rise-as-labs-proliferate-with-scantregulation.html

Guldenmund, F. W. (2000). The nature of safety culture: a review of theory and research. Safety Science, 34(1-3), 215-257. Http://doi.org/10.1016/S0925-7535(00)00014-X

Haberman, A. (2014). Report on College of Engineering Safety Culture Survey (p. 43). University of Florida. Retrieved from https://www.eng.ufl.edu/labsafety/wp-content/uploads/sites/28/2014/09/Safety-CultureSurvey-Report.pdf

Hancock, R. (2014). Lab Management: A Capabilities-Based Approach. Professional Safety; Des Plaines, 59(12), $44-45$.

Haq, G., \& Paul, A. (2013). Environmentalism Since 1945. Routledge.

Harvey, J., Erdos, G., Bolam, H., Cox, M. A. A., Kennedy, J. N. P., \& Gregory, D. T. (2002). An analysis of safety culture attitudes in a highly regulated environment. Work \& Stress, 16(1), 18-36.

Http://doi.org/10.1080/02678370110113226

Haybaeck, J., Heikenwalder, M., Klevenz, B., Schwarz, P., Margalith, I., Bridel, C., ... Aguzzi, A. (2016). Canadian Biosafety Handbook Second Edition. (D. Westaway, Ed.) (Vol. 7). Retrieved from http://dx.plos.org/10.1371/journal.ppat.1001257

Health and Safety Authority. (2012). Storage of Hazardous Chemicals in Laboratories - Health and Safety Authority. Retrieved August 17, 2016, from 
http://www.hsa.ie/eng/Publications_and_Forms/Publications/Chemical_and_Hazardous_Substances/Storag e_of_Hazardous_Chemicals_in_Laboratories.html

Health and Safety Authority. (n.d.). Lone Workers - Health and Safety Authority. Retrieved September 1, 2016, from http://www.hsa.ie/eng/Topics/Hazards/Lone_Workers/

Health and Safety Laboratory Health and Safety Guidelines, 4th Edition. (n.d.). Retrieved February 22, 2017, from http://www.acpo.on.ca/resources/health-safety/laboratory_e.php

Health Canada. (2009, April 29). WHMIS Quick Facts - Storage Requirements [Health Canada, 2008] [publication]. Retrieved December 29, 2016, from http://www.hc-sc.gc.ca/ewh-semt/pubs/occup-travail/storageentreposage/index-eng.php

Health Canada. (2015, February 27). WHMIS 2015. Retrieved February 19, 2017, from http://www.hcsc.gc.ca/ewh-semt/occup-travail/whmis-simdut/ghs-sgh/index-eng.php

Hill Jr., R. H. (2007). The emergence of laboratory safety. Journal of Chemical Health and Safety, 14(3), 14-19. Https://doi.org/10.1016/j.jchas.2006.10.001

Hill Jr., R. H. (2010). GHS and its impact on laboratory safety. Journal of Chemical Health and Safety, 17(4), 5-11. Https://doi.org/10.1016/j.jchas.2009.09.002

Hill Jr, R. H., Crumrine, D. S., Doemeny, L. J., Fleming, S. A., Fivizzani, K. P., Hausner, D. B., ... Gmurczyk, M. U. (2012). Creating Safety Cultures in Academic Institutions (first). Retrieved from https:/www.acs.org/content/dam/acsorg/about/governance/committees/chemicalsafety/academic-safetyculture-report-final-v2.pdf

Hill, R. H., \& Finster, D. C. (2013). Academic leaders create strong safety cultures in colleges and universities. Journal of Chemical Health and Safety, 20(5), 27-34.

Hill, R. H., \& Finster, D. C. (2016). Laboratory Safety for Chemistry Students. John Wiley \& Sons.

Hofstein, A., \& Lunetta, V. N. (2004). The laboratory in science education: Foundations for the twenty-first century. Science Education, 88(1), 28-54. Http://doi.org/10.1002/sce.10106

Hoff, D. J. (2003). Science-lab safety upgraded after mishaps. Education Week. Retrieved from http://campuses.fortbendisd.com/campuses/documents/teacher/2008/teacher_20080903_1604.pdf

Höppe, P. (2002). Different aspects of assessing indoor and outdoor thermal comfort. Energy and Buildings, 34(6), 661-665. Https://doi.org/10.1016/S0378-7788(02)00017-8 
Hu, J. (2011, April 8). Plague death uncovered. The Chicago Maroon. Retrieved from http://chicagomaroon.com/2011/04/08/plague-death-uncovered/

Huising, R., \& Silbey, S. S. (2011). Governing the gap: Forging safe science through relational regulation. Regulation \& Governance, 5(1), 14-42.

Integrated Risk Management. (2011, November). Environmental Health and Safety (EHS) Management System Policy [Academic]. Retrieved April 21, 2017, from http://www.ryerson.ca/policies/board/ehsmanagementsystempolicy/

Iowa State University. (2011). Laboratory Safety Manual (2nd ed.). Retrieved from http://polycomp.mse.iastate.edu/files/2011/06/Iowa-State-Lab-Safety-Manual.pdf

Ismail, I. S., Rahman, S. B. A., Noordin, N. M., Mustafa, S. M. S., Sedghpour, B. S., Sabbaghan, M., \& Sataei, F. M. (2013). 6th International Conference on University Learning and Teaching (incult 2012)A Survey on the Pre Service Chemistry Teachers' Lab Safety Education. Procedia - Social and Behavioral Sciences, 90, 57-62. Http://doi.org/10.1016/j.sbspro.2013.07.065

Jenkins, P. L., Phillips, T. J., Mulberg, E. J., \& Hui, S. P. (1992). Activity patterns of Californians: use of and proximity to indoor pollutant sources. Atmospheric Environment. Part A. General Topics, 26(12), 21412148.

Jessop, R. (2012). Coinage of the Term Environment: A Word Without Authority and Carlyle's Displacement of the Mechanical Metaphor. Literature Compass, 9(11), 708-720. Http://doi.org/10.1111/j.17414113.2012.00922.x

Jeskie, K. B., Ashbrook, P., Casadonte, D., Decker, D., Doemeny, L. J., Hill, R. H., ... Gmurczyk, M. U. (2015). Identifying and Evaluating Hazards in Research Laboratories. Retrieved from https:/www.acs.org/content/dam/acsorg/about/governance/committees/chemicalsafety/publications/identif ying-and-evaluating-hazards-in-research-laboratories.pdf

Jia, H. (2016, January 18). Deadly accident sounds alarm for safety in Chinese labs. Retrieved November 8, 2016 , from https:/www.chemistryworld.com/news/deadly-accident-sounds-alarm-for-safety-in-chineselabs/9350.article

Jing, G. (2015, December 12). China’s Laboratory Safety Must be Highlighted, Improved. Retrieved July 16, 2016, from http://english.cri.cn/12394/2015/12/26/2743s910192.htm 
Johnson, J., \& Kemsley, J. (2011). Academic Lab Safety Under Exam | October 24, 2011 Issue - Vol. 89 Issue 43 | Chemical \& Engineering News, 89(43), 25-27.

Kaiser, J. (2011, September 12). Updated: University of Chicago Microbiologist Infected From Possible Lab Accident. Science | AAAS. Retrieved from http://www.sciencemag.org/news/2011/09/updated-universitychicago-microbiologist-infected-possible-lab-accident

Karapantsios, T. D., Boutskou, E. I., Touliopoulou, E., \& Mavros, P. (2008). Evaluation of chemical laboratory safety based on student comprehension of chemicals labelling. Education for Chemical Engineers, 3(1), e66-e73. Https://doi.org/10.1016/j.ece.2008.02.001

Kaufman, J. A. (1978). Safety in the academic laboratory. Http://doi.org/10.1021/ed055pa337

Keller, E. (1910). The Hygiene of the Small Chemical Laboratory. Journal of Industrial \& Engineering Chemistry, 2(6), 246-251. Https://doi.org/10.1021/ie50018a005

Kemsley, J. N. (2009). Negligence Caused UCLA Death | Latest News | Chemical \& Engineering News, 87, 7. Kemsley, J. (2012). Dow Chemical Teams Up with Universities On Laboratory Safety | October 29, 2012 Issue Vol. 90 Issue 44 | Chemical \& Engineering News, 90(44), 21-23.

Kemsley, J. (2013). Survey Exposes Lab Safety Gaps | January 21, 2013 Issue - Vol. 91 Issue 3 | Chemical \& Engineering News, 91(3), 30-31.

Lab bench. (2003, 2008). Thefreedictionary.com. Farlex clipart collection. Retrieved from http://www.thefreedictionary.com/lab+bench

Laboratory Safety Institute. (2014). Memorial Wall. Retrieved November 2, 2016, from http://www.labsafetyinstitute.org/memorialwall.html

Lab Safety Culture Survey - Draft Report. (2012). Retrieved from http://www.bioraft.com/lab-safety-culture-surveydraft-report

Laboratory Facilities. (2011). National Academies Press (US). Retrieved from https://www.ncbi.nlm.nih.gov/books/NBK55867/

Langerman, N. (2009a). Laboratory safety? Journal of Chemical Health and Safety, 16(3), 49-50. Http://doi.org/10.1016/j.jchas.2009.03.004

Langerman, N. (2009b). Lab-scale process safety management. Journal of Chemical Health and Safety, 16(4), 2228. Http://doi.org/10.1016/j.jchas.2008.09.013 
Langerman, N. (2011). Deaths Continue. Journal of Chemical Health and Safety, 18(4), 38-39. Https://doi.org/10.1016/j.jchas.2011.05.004

Langerman, N. (2012). Legislate safety? Journal of Chemical Health and Safety, 19(5), 64-65.

Https://doi.org/10.1016/j.jchas.2012.07.005

Laurent, A., Perrin, L., \& Dufaud, O. (2014). Investigation and Analysis of an explosion in a Research Laboratory at a French University. The Italian Association of Chemical Engineering, 36. Http://doi.org/10.3303/CET1436002

Lee, S. C., Li, W.-M., \& Chan, L. Y. (2001). Indoor air quality at restaurants with different styles of cooking in metropolitan Hong Kong. Science of the Total Environment, 279(1), 181-193.

Lee, S.-C., Guo, H., Li, W.-M., \& Chan, L.-Y. (2002). Inter-comparison of air pollutant concentrations in different indoor environments in Hong Kong. Atmospheric Environment, 36(12), 1929-1940.

Lowry, G. (1978). A university-level course in laboratory safety. Http://doi.org/10.1021/ed055pa263

Mcgarry, K. A., Hurley, K. R., Volp, K. A., Hill, I. M., Merritt, B. A., Peterson, K. L., ... Tolman, W. B. (2013). Student Involvement in Improving the Culture of Safety in Academic Laboratories. Journal of Chemical Education, 90(11), 1414-1417. Http://doi.org/10.1021/ed400305e

Mcleod, V. (2011b, September 9). Laboratory Hazards and Risks. Retrieved July 28, 2016, from http://www.labmanager.com/lab-health-and-safety/2011/09/laboratory-hazards-and-risks

Mcleod, V. (2010, December 9). Biosafety Levels 1, 2, 3 \& 4. Retrieved November 21, 2016, from http:/www.labmanager.com/lab-health-and-safety/2010/12/biosafety-levels-1-2-3-4

Mcleod, V. (2011b, September 9). Laboratory Hazards and Risks. Retrieved February 22, 2017, from http://www.labmanager.com/lab-health-and-safety/2011/09/laboratory-hazards-and-risks

Mcmaster University. (2008). Laboratory Safety Handbook. Retrieved from http://www.workingatmcmaster.ca/med/document/Lab-Safety-Handbook-1-36.pdf

Mearns, K., \& Flin, R. (1999). Assessing the state of organizational safety-culture or climate? Current Psychology, 18(1), 5-17. Https://doi.org/10.1007/s12144-999-1013-3

Meiping, Y. (2016, September 22). 3 students injured after blast in lab. Retrieved from http://www.shanghaidaily.com/metro/society/3-students-injured-after-blast-in-lab/shdaily.shtml

Michalovic, M. (2008). Not-So-Great moments in chemical safety. Chemical Heritage News Magazine, 26(2). 
Miller, J. M., Astles, R., Baszler, T., Chapin, K., Carey, R., Garcia, L., ... Wiedbrauk, D. (2012). Guidelines for Safe Work Practices in Human and Animal Medical Diagnostic Laboratories. Retrieved from http://www.cdc.gov/mmwr/preview/mmwrhtml/su6101a1.htm

Miller, S. (2014). Dalhousie Green Labs: Increasing Solid Waste Diversion in Dalhousie Laboratories. Retrieved from https://www.google.ca/?Gfe_rd=cr\&ei=mnyav6p_jcwh8wfvv62qbq\#q=Miller\%2C+S.+(2014).+Dalhousie +Green+Labs:+Increasing+Solid+Waste+Diversion+in+Dalhousie+Laboratories.+

Ministry of Labour. (2009, June). Chapter 1. Overview of WHMIS | Workplace Hazardous Materials Information System: A Guide to the Legislation | Ministry of Labour. Retrieved from https://www.labour.gov.on.ca/english/hs/pubs/whmis/whmis_1.php

Ministry of Labour. (2015, December 3). Personal Protective Equipment: Occupational Health and Safety Guidelines for Farming Operations in Ontario | Ontario Ministry of Labour. Retrieved November 21, 2016, from https://www.labour.gov.on.ca/english/hs/pubs/farming/gl_ppe.php\#definition

Ministry of Labour. (2016, March 15). Explosion Risk Associated with Old Laboratory Samples | Ministry of Labour. Retrieved November 22, 2016, from https:/www.labour.gov.on.ca/english/hs/pubs/alerts/a35.php Mohammed, E., Korsa, H. A., Soltan, H., \& Roshdy, M. (2015). Research Journal of Medicine and Medical Sciences. Research Journal of Medicine and Medical Sciences, 10(2), 7-18.

The National Academies of Science, Engineering, and Medicine. (2014, July 31). Strong, Positive Safety Culture in Chemical Labs Requires Support from All Levels Within Research Institutions [News]. Retrieved July 23, 2016, from http://www8.nationalacademies.org/onpinews/newsitem.aspx?Recordid=18706

Nara, L. (2016). Process Safety Begins in the School Laboratory. Chemical Engineering Progress, 112(2), $21-21$.

Neal, A., \& Griffin, M. A. (2002). Safety climate and safety behaviour. Australian Journal of Management, 27(1 suppl), 67-75.

Occupational Health and Safety Act, R.S.O. 1990, c. O.1, §§ VIII-55-66 (Government of Ontario 2016)

Ontario Ministry of Labour. (2008, August). Chapter 1. Overview of WHMIS | Workplace Hazardous Materials Information System: A Guide to the Legislation | Ministry of Labour [Government website]. Retrieved July 29, 2016, from https://www.labour.gov.on.ca/english/hs/pubs/whmis/whmis_1.php

Ontario Ministry of Labour. (2013, October). The Occupational Health and Safety Act: faqs | Ministry of Labour 
[Government website]. Retrieved July 29, 2016, from

https://www.labour.gov.on.ca/english/hs/faqs/ohsa.php

Ontario Ministry of Labour. (2015a, March 20). Introduction: A Guide to the Occupational Health and Safety Act| Ministry of Labour. Retrieved February 19, 2017, from

https://www.labour.gov.on.ca/english/hs/pubs/ohsa/ohsag_intro.php

Ontario Ministry of Labour. (2015b, March 20). The Internal Responsibility System: A Guide to the Occupational Health and Safety Act| Ministry of Labour. Retrieved February 23, 2017, from https://www.labour.gov.on.ca/english/hs/pubs/ohsa/ohsag_irs.php

Ontario, G. Of. (2014, July). Law Document English View [Text]. Retrieved July 29, 2016, from https://www.ontario.ca/laws/view

Olewski, T., Ahammad, M., Quraishy, S., Gan, N., \& Vechot, L. (2016). Building process safety culture at Texas A\&M University at Qatar: A case study on experimental research. Journal of Loss Prevention in the Process Industries, 44, 642-652. Https://doi.org/10.1016/j.jlp.2016.08.022

Oxford University Press. (n.d.). Environment. Oxford Dictionaries. Retrieved from http://www.oxforddictionaries.com/definition/english/environment

Petropedia. (n.d.). What is Chemical Inventory (CIS)? - Definition from Petropedia. Retrieved November 22, 2016, from https://www.petropedia.com/definition/5266/chemical-inventory-cis

Pesta, S., \& Kaufman, J. A. (1986). Laboratory safety in academic institutions. J. Chem. Educ, 63(10), A242.

Pipitone, D. A. (Ed.). (1991). Safe Storage of Laboratory Chemicals (2nd ed.). John Wiley \& SONS, INC.

Pons, G., Rogers, C., \& Robinson, D. A. (2013). Lab Coat Management Program. Retrieved from https://ehs.umass.edu/sites/default/files/Campus\%20Lab\%20Coat\%20Management\%20\%20Program\%20F all\%202014.pdf

Public Health Agency of Canada. (2005, January 5). Chapter 9: Biological Safety Cabinets - Laboratory Biosafety Guidelines: 3rd Edition 2004. Retrieved February 23, 2017, from http://www.phac-aspc.gc.ca/publicat/lbgldmbl-04/ch9-eng.php

Public Health Agency of Canada. (2004). Laboratory Biosafety Guidelines 3rd Edition 2004 - Pathogen Regulation Directorate - Public Health Agency of Canada (3rd ed.). Canada. Retrieved from http://www.phacaspc.gc.ca/publicat/lbg-ldmbl-04/pdf/lbg_2004_e.pdf 
Public Health Agency of Canada. (2005, January 5). Chapter 2: Biological Safety - Laboratory Biosafety Guidelines: 3rd Edition 2004. Retrieved July 28, 2016, from http://www.phac-aspc.gc.ca/publicat/lbg1dmb1-04/ch2-eng.php

Public Health Agency of Canada Government of Canada. (2016, January 29). Canadian Biosafety Handbook, Second Edition - Canadian Biosafety Standards and Guidelines. Retrieved November 8, 2016, from http://canadianbiosafetystandards.collaboration.gc.ca/cbh-gcb/index-eng.php

Queen's University Environmental Health \& Safety. (2013, March 14). Lab coat. Retrieved from http://www.safety.queensu.ca/safety/policy/eh\&s/labcoat.pdf

Rahman, A. (2010, June 2). Workshops, manual...Delhi University looks anew at lab safety. The Hindustan Times; New Delhi. New Delhi, India. Retrieved from http://search.proquest.com.ezproxy.lib.ryerson.ca/docview/356810431/citation/53C8A776B7F74DFFPQ/1

Rengarajan, K. (2012). Laboratory self-inspection program participation as an indication of improved safety culture at Emory University. Journal of Chemical Health and Safety. Https://doi.org/10.1016/j.jchas.2012.02.003

Reeger, J. (2012, October 26). Chemistry lab mishaps in academic settings happen frequently. Retrieved July 5, 2016, from http://riblive.com/home/2834482-74/safety-chemistry-lab-accidents-kaufman-incidentslangerman-students-teacher-based

Reinhardt, P. A., Ashbrook, P. C., \& Leonard, K. L. (1995). Pollution Prevention and Waste Minimization in Laboratories. CRC Press.

Reis, R. K., Gir, E., \& Canini, S. R. M. S. (2004). Accidents with biological material among undergraduate nursing students in a public Brazilian university. Brazilian Journal of Infectious Diseases, 8(1), 18-24. Https://doi.org/10.1590/S1413-86702004000100003

Reniers, G. L. L., Ponnet, K., \& Kempeneers, A. (2014). Higher education chemical lab safety interventions: Efficacious or ineffective? Journal of Chemical Health and Safety, 21(1), 4-8. Http://doi.org/10.1016/j.jchas.2013.09.001

Richards-Babb, M., Bishoff, J., Carver, J. S., Fisher, K., \& Robertson-Honecker, J. (2010). Keeping it safe: Chemical safety in the high school laboratory. Journal of Chemical Health and Safety, 17(1), 6-14. Https://doi.org/10.1016/j.jchas.2009.05.001

Rosenstock, I., Strecher, V., \& Becker, M. (1988b). Social Learning Theory and the Health Belief Model. Health 
Education \& Behaviour, 15(2), 175-183. Https://doi.org/10.1177/109019818801500203

Ruffing, A. A. (2013). Personal Protective Equipment and Laboratory Safety Training: The Roles of Attitude, Subjective Norm, and Perceived Control.

Rugani, L., \& Dickson, C. (2014, July 31). Home | The National Academies of Sciences, Engineering, and Medicine | National-Academies.org | Where the Nation Turns for Independent, Expert Advice. Retrieved July 28, 2016, from http://www8.nationalacademies.org/onpinews/newsitem.aspx?Recordid=18706

Safeopedia. (n.d.). What is Environmental Health and Safety (EH\&S) (EHS)? - Definition from Safeopedia. Retrieved January 12, 2017, from https://www.safeopedia.com/definition/1390/environmental-health-andsafety-ehs-ehs

Scheid, P. (2015). Viruses in close associations with free-living amoebae. Parasitology Research, 114(11), 39593967. Https://doi.org/10.1007/s00436-015-4731-5

School Chemistry Laboratory Safety Guide. (2006). Retrieved from: https://www.cdc.gov/niosh/docs/2007107/pdfs/2007-107.pdf

Schröder, I., Huang, D. Y. Q., Ellis, O., Gibson, J. H., \& Wayne, N. L. (2016). Laboratory safety attitudes and practices: A comparison of academic, government, and industry researchers. Journal of Chemical Health and Safety, 23(1), 12-23. Https://doi.org/10.1016/j.jchas.2015.03.001

Sedghpour, B. S., Sabbaghan, M., \& Sataei, F. M. (2013). A Survey On The Pre Service Chemistry Teachers' Lab Safety Education. Procedia-Social and Behavioral Sciences, 90, 57-62.

Sewell, D. L. (1995). Laboratory-associated infections and biosafety. Clinical Microbiology Reviews, 8(3), 389405.

Shariff, A. M., \& Norazahar, N. (2012). At-risk behaviour analysis and improvement study in an academic laboratory. Safety Science, 50(1), 29-38.

Simpson, K. M. (2005). Laboratory Manual. Retrieved from http://chemmybear.com/ucr2005/CHEM001CLabManual_05.pdf

Skvorc, C., \& Wilson, D. E. (2015). Criminal Prosecution-A Jumpstart for Laboratory Safety Accountability? Applied Biosafety, 20(3), 136-141. Https://doi.org/10.1177/153567601502000303

Speare, R. J. (2015, May 21). Second edition of the Canadian Biosafety Standard | Research | Research | UPEI. Retrieved November 8, 2016, from http:/www.upei.ca/research/notice/canadian-biosafety-standard 
Staehle, I. O., Chung, T. S., Stopin, A., Vadehra, G. S., Hsieh, S. I., Gibson, J. H., \& Garcia-Garibay, M. A. (2015). An Approach to Enhance the Safety Culture of an Academic Chemistry Research Laboratory by Addressing Behavioral Factors. ACS Publications, 217-222. Http://doi.org/10.1021/acs.jchemed.5b00299 Standfor University. (2014). Advancing Safety Culture in the University Laboratory A report of the Task Force for Advancing the Culture of Laboratory at Stanford University. Retrieved from https://web.stanford.edu/dept/EHS/cgi-bin/lsctf/

Stroud, L. M., Stallings, C., \& Korbusieski, T. J. (2007). Implementation of a science laboratory safety program in North Carolina schools. Journal of Chemical Health and Safety, 14(3), 20-30.

Stoye, E. (2016, March 23). University of Hawaii researcher loses arm in lab explosion | Chemistry World. Retrieved July 16, 2016, from http://www.rsc.org/chemistryworld/2016/03/hawaii-university-accidentresearcher-loses-arm

Sulkin, S. E., \& Pike, R. M. (1951). Survey of Laboratory-Acquired Infections*†. American Journal of Public Health and the Nations Health, 41(7), 769-781.

Tenkate, T., mcbride, J., \& Richard, M. (2001). Development and trial of a chemical hazard assessment scheme for university laboratories. Journal of occupational health and safety Australia and New Zealand, 17(2), 147152.

The Hazard Identification and Evaluation Task Force of the American Chemical Society’s Committee on Chemical Safety. (2015). Identifying and Evaluating Hazards in Research Laboratories. Retrieved from https://www.acs.org/content/dam/acsorg/about/governance/committees/chemicalsafety/publications/identif ying-and-evaluating-hazards-in-research-laboratories.pdf

TSI Incorporated. (2014). LABORATORY DESIGN HANDBOOK. Retrieved from http://www.tsi.com/uploadedfiles/_Site_Root/Products/Literature/Handbooks/2980330Clabcontrolshandbook.pdf

Trager, R. (2009, January 23). UCLA lab assistant dies | News. Chemistry World. Retrieved from https://www.chemistryworld.com/news/ucla-lab-assistant-dies/3004085.article

Trager, R. (2014, June 26). UCLA chemist avoids prison time for lethal lab accident. Chemistry World. Retrieved from https://www.chemistryworld.com/news/ucla-chemist-avoids-prison-time-for-lethal-labaccident/7501.article 
The SCOTSMAN. (2013, October 3). Man hurt after Edinburgh chemical lab accident. Retrieved from http://www.scotsman.com/news/education/man-hurt-after-edinburgh-chemical-lab-accident-1-3124199

Transportation Safety Board of Canada. (2014, August 19). Lac-Mégantic runaway train and derailment investigation summary - Railway Investigation Report R13D0054 - Transportation Safety Board of Canada. Retrieved November 26, 2016, from http://www.tsb.gc.ca/eng/rapportsreports/rail/2013/r13d0054/r13d0054-r-es.asp

Twisselmann, B. (2003). The Discovery of the Germ. BMJ : British Medical Journal, 327(7405), 57.

University of California Riverside. (n.d.). Laboratory safety manual: Department of Biochemistry. Retrieved from http://www.ehs.ucr.edu/laboratory/laboratorysafetymanualbiochemistry.pdf

University of California Santa Barbara. (2014). Laboratory Accidents | Environmental Health \& Safety. Retrieved November 2, 2016, from http://www.ehs.ucsb.edu/labsafety/laboratory-accidents

University of Florida. (2016). Lab Safety Checklist» Environmental Health \& Safety. Retrieved October 17, 2016, from http://www.ehs.ufl.edu/programs/lab/lssurvey/checklist/

University of Guelph. (2016). Laboratory Safety Manual (4th ed.). Retrieved from https:/www.uoguelph.ca/hr/system/files/Lab\%20Safety\%20Manual\%20April\%202016.pdf

University of Iowa. (2012, July 17). Chemical Storage: Nine Compatible Storage Group System | Environmental Health and Safety. Retrieved November 22, 2016, from https://ehs.research.uiowa.edu/chemical-storagenine-compatible-storage-group-system

University of Memphis. (n.d.). Laboratory Safety Checklist Chemical. Retrieved from http:/www.memphis.edu/ehs/pdfs/labsafetychklistchem.pdf

University of Washington Environmental Health \& Safety. (2015). Appendix E-checklists. Retrieved from http://www.ehs.washington.edu/manuals/1sm/lsme.pdf

University of Iowa. (2012, July 17). Chemical Storage: Nine Compatible Storage Group System | Environmental Health and Safety. Retrieved November 22, 2016, from https://ehs.research.uiowa.edu/chemical-storagenine-compatible-storage-group-system

University of Nebraska-Lincoln. (2015, December). Biosafety | Environmental Health \& Safety | University of Nebraska-Lincoln. Retrieved June 28, 2016, from http://ehs.unl.edu/sop/biosafety

University of Sussex. (2012). Management of Lone Working Risks Policy (No. 9). Retrieved from 
https://www.sussex.ac.uk/webteam/gateway/file.php?Name=lone-working-policy.pdf\&site=21

University of Twente. (2017, February 27). Health Belief Model. Retrieved December 5, 2016, from

https://www.utwente.nl/cw/theorieenoverzicht/Theory\%20Clusters/Health\%20Communication/Health_Beli ef_Model/

U.S. Chemical Safety Board. (2011). CSB Releases Investigation into 2010 Texas Tech Laboratory Accident; Case Study Identifies Systemic Deficiencies in University Safety Management Practices - Investigations - News | the U.S. Chemical Safety Board. Retrieved from http://www.csb.gov/csb-releases-investigation-into-2010texas-tech-laboratory-accident-case-study-identifies-systemic-deficiencies-in-university-safetymanagement-practices/

US EPA. (2016, June 28). About the TSCA Chemical Substance Inventory [Overviews and Factsheets]. Retrieved November 22, 2016, from https://www.epa.gov/tsca-inventory/about-tsca-chemical-substance-inventory U.S. National Institutes of Health. (n.d.). Understanding the Proper Use of Fire Doors. Retrieved December 6, 2016, from https://www.ors.od.nih.gov/News/Pages/understanding_use_fire_door.aspx

U.S. National Research Council. (1995). Prudent Practices in the Laboratory: Handling and Disposal of Chemicals. Retrieved from https://www.nap.edu/read/4911/chapter/9

U.S. National Research Council. (2014). Safe Science: Promoting a Culture of Safety in Academic Chemical Research. Retrieved from https://www.nap.edu/catalog/18706/safe-science-promoting-a-culture-of-safetyin-academic-chemical

U.S. National Research Council. (2014). Safe Science: Promoting a Culture of Safety in Academic Chemical Research. Washington, D.C.: National Academies Press. Retrieved from http://www.nap.edu/catalog/18706 U.S. National Research Council. (2011). Environmental Health and Safety Management System. National Academies Press (US). Retrieved from https://www.ncbi.nlm.nih.gov/books/NBK55873/

U.S. National Research Council (US) (2011). The Culture of Laboratory Safety. National Academies Press (US). Retrieved from https://www.ncbi.nlm.nih.gov/books/NBK55882/

Van Noorden, R. (2011). A death in the lab. Nature News, 472(7343), 270-271. Https://doi.org/10.1038/472270a Van Noorden, R. (2013). Safety survey reveals lab risks. Nature, 493(7430), 9-10. Http://doi.org/10.1038/493009a

Vision, Y. (2012, February 2). Fire reported at Chemistry department. Retrieved from http://www.yorkvision.co.uk/news/fire-reported-at-chemistry-department/02/02/2012 
Vergano, D., \& Korte, G. (2011, October 19). Report finds fault with college labs over poor safety record [news]. Retrieved November 1, 2016, from http://www.usatoday.com/tech/science/story/2011-10-19/college-labsafety-chemistry/50818666/1

Virginia University. (n.d.). Risk Groups and Biosafety Levels, UVA. Retrieved November 21, 2016, from http://ehs.virginia.edu/biosafety/bio.riskgroups.html

Walters, D. B., \& Keith, L. H. (2007). Chemists helping chemists-The Environmental \& Chemical Safety Educational Institute. Journal of Chemical Health and Safety, 14(3), 4. Https://doi.org/10.1016/j.jchas.2007.03.005

Wargniez, A. B., Oleas, R. C., \& Yamaguchi, K. S. (2012). Improving laboratory safety through mini-scale experiments: A case study of New Jersey City University. Journal of Chemical Health and Safety, 19(6), $12-23$.

Washington DC. (n.d.). Laboratory safety checklist your laboratory and the occupational safety \& health act (OSHA). Retrieved from file://Users/irm/Downloads/oshalabsafetychecklist_120412.pdf

Watch, D., Tolat, D., \& mcnay, G. (2010, May 26). Academic Laboratory. Retrieved July 28, 2016, from http://www.wbdg.org/design/academic_lab.php

Watson, N. (2013, March 1). Laboratory Safety Culture Survey - Lifting the Veil. Retrieved August 17, 2016, from http://www.bioraft.com/blog/laboratory-safety-culture-survey-lifting-veil

Weil, m. W. (2012, February 6). Information, communication, and culture in the management of academic laboratory chemicals. University of Massachusetts Lowell. Retrieved from

http://search.proquest.com/docview/1241415431

Whole Building Design Guide Staff. (2010, May 26). Laboratory: Wet | Whole Building Design Guide. Retrieved November 22, 2016, from https://www.wbdg.org/design/lab_wet.php

Wu, T.-C., Liu, C.-W., \& Lu, M.-C. (2007). Safety climate in university and college laboratories: Impact of organizational and individual factors. Journal of Safety Research, 38(1), 91-102.

Workplace Safety North. (2015, June 2). Top 7 significant changes to federal WHMIS law. Retrieved October 29, 2016, from https://www.workplacesafetynorth.ca/news/news-post/top-7-significant-changes-federal-whmis-law Worksafe BC. (2008). Laboratory health and safety handbook. Retrieved from http://www.mtpinnacle.com/pdfs/labsafetymanual1.pdf 
World Health Organization. (2004). Laboratory biosafety manual (3rd ed.). Geneva. Retrieved from http://www.who.int/csr/resources/publications/biosafety/en/Biosafety7.pdf

Wu, T.-C., Chen, C.-H., \& Li, C.-C. (2008). A correlation among safety leadership, safety climate and safety performance. Journal of Loss Prevention in the Process Industries, 21(3), 307-318.

Https://doi.org/10.1016/j.jlp.2007.11.001

Wu, T.-C., Chang, S.-H., Shu, C.-M., Chen, C.-T., \& Wang, C.-P. (2011). Safety leadership and safety performance in petrochemical industries: The mediating role of safety climate. Journal of Loss Prevention in the Process Industries, 24(6), 716-721. Https://doi.org/10.1016/j.jlp.2011.04.007

Wyllie, R., Lee, K., Morris-Benavides, S., \& Matos, B. (2016). What to expect when you're inspecting: A summary of academic laboratory inspection programs. Journal of Chemical Health and Safety, 23(2), 18-24. 